\title{
Measurement of the muon reconstruction performance of the ATLAS detector using 2011 and 2012 LHC proton-proton collision data
}

\author{
ATLAS Collaboration ${ }^{\star}$ \\ CERN, 1211 Geneva 23, Switzerland
}

Received: 16 July 2014 / Accepted: 14 October 2014 / Published online: 26 November 2014

(C) CERN for the benefit of the ATLAS collaboration 2014. This article is published with open access at Springerlink.com

\begin{abstract}
This paper presents the performance of the ATLAS muon reconstruction during the LHC run with $p p$ collisions at $\sqrt{s}=7-8 \mathrm{TeV}$ in 2011-2012, focusing mainly on data collected in 2012. Measurements of the reconstruction efficiency and of the momentum scale and resolution, based on large reference samples of $J / \psi \rightarrow \mu \mu, Z \rightarrow \mu \mu$ and $\Upsilon \rightarrow \mu \mu$ decays, are presented and compared to Monte Carlo simulations. Corrections to the simulation, to be used in physics analysis, are provided. Over most of the covered phase space (muon $|\eta|<2.7$ and $5 \lesssim p_{\mathrm{T}} \lesssim 100 \mathrm{GeV}$ ) the efficiency is above $99 \%$ and is measured with per-mille precision. The momentum resolution ranges from $1.7 \%$ at central rapidity and for transverse momentum $p_{\mathrm{T}} \simeq 10 \mathrm{GeV}$, to $4 \%$ at large rapidity and $p_{\mathrm{T}} \simeq 100 \mathrm{GeV}$. The momentum scale is known with an uncertainty of $0.05 \%$ to $0.2 \%$ depending on rapidity. A method for the recovery of final state radiation from the muons is also presented.
\end{abstract}

\section{Introduction}

The efficient identification of muons and the accurate measurement of their momenta are two of the main features of the ATLAS detector [1] at the LHC. These characteristics are often crucial in physics analysis, as for example in precise measurements of Standard Model processes [2-4], in the discovery of the Higgs boson, in the determination of its mass [5,6], and in searches for physics beyond the Standard Model $[7,8]$. This publication presents the performance of the ATLAS muon reconstruction during the LHC run at $\sqrt{s}=7-8 \mathrm{TeV}$, focusing mainly on data collected in 2012. The performance of the ATLAS muon reconstruction has already been presented in a recent publication [9] based on 2010 data. The results presented here are based on

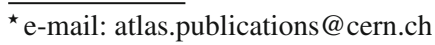

an integrated luminosity $\approx 500$ times larger, which allows a large reduction of the uncertainties. The measurements of the efficiency, of the momentum scale and resolution are discussed with a particular emphasis on the comparison between data and Monte Carlo (MC) simulation, on the corrections used in the physics analyses and on the associated systematic uncertainties. Muons with very large transverse momentum, ${ }^{1}$ $p_{\mathrm{T}}>120 \mathrm{GeV}$, are not treated here as they will be the subject of a forthcoming publication on the alignment of the ATLAS muon spectrometer and its high- $p_{\mathrm{T}}$ performance.

This publication is structured as follows: Sect. 2 gives a short description of muon detection in ATLAS and Sect. 3 describes the real and simulated data samples used in the performance analysis. The measurement of the reconstruction efficiency is described in Sect. 4 while Sect. 5 reports the momentum scale and resolution. A method for including photons from final-state radiation in the reconstruction of the muon kinematics, is described in Sect. 6. Conclusions are given in Sect. 7.

\section{Muon identification and reconstruction}

A detailed description of the ATLAS detector can be found elsewhere [1]. The ATLAS experiment uses the information from the muon spectrometer (MS) and from the inner detector (ID) and, to a lesser extent, from the calorimeter, to identify and precisely reconstruct muons produced in the $p p$ collisions.

\footnotetext{
1 ATLAS uses a right-handed coordinate system with its origin at the nominal interaction point (IP) in the centre of the detector and the $z$-axis along the beam pipe. The $x$-axis points from the IP to the centre of the LHC ring, and the $y$-axis points upward. Cylindrical coordinates $(r, \phi)$ are used in the transverse plane, $\phi$ being the azimuthal angle around the beam pipe. The pseudorapidity and the transverse momentum are defined in terms of the polar angle $\theta$ as $\eta=-\ln \tan (\theta / 2)$ and $p_{\mathrm{T}}=$ $p \sin \theta$, respectively. The $\eta-\phi$ distance between two particles is defined as $\Delta R=\sqrt{\Delta \eta^{2}+\Delta \phi^{2}}$.
} 
The MS is the outermost of the ATLAS sub-detectors: it is designed to detect charged particles in the pseudorapidity region up to $|\eta|=2.7$, and to provide momentum measurement with a relative resolution better than $3 \%$ over a wide $p_{\mathrm{T}}$ range and up to $10 \%$ at $p_{\mathrm{T}} \approx 1 \mathrm{TeV}$. The MS consists of one barrel part (for $|\eta|<1.05$ ) and two end-cap sections. A system of three large superconducting air-core toroid magnets provides a magnetic field with a bending integral of about $2.5 \mathrm{Tm}$ in the barrel and up to $6 \mathrm{Tm}$ in the end-caps. Triggering and $\eta, \phi$ position measurements, with typical spatial resolution of 5-10 $\mathrm{mm}$, are provided by the Resistive Plate Chambers (RPC, three doublet layers for $|\eta|<1.05$ ) and by the Thin Gap Chambers (TGC, three triplet and doublet layers for $1.0<|\eta|<2.4$ ). Precise muon momentum measurement is possible up to $|\eta|=2.7$ and it is provided by three layers of Monitored Drift Tube Chambers (MDT), each chamber providing six to eight $\eta$ measurements along the muon track. For $|\eta|>2$ the inner layer is instrumented with a quadruplet of Cathode Strip Chambers (CSC) instead of MDTs. The single hit resolution in the bending plane for the MDT and the CSC is about $80 \mu \mathrm{m}$ and $60 \mu \mathrm{m}$, respectively. Tracks in the MS are reconstructed in two steps: first local track segments are sought within each layer of chambers and then local track segments from different layers are combined into full MS tracks.

The ID provides an independent measurement of the muon track close to the interaction point. It consists of three subdetectors: the Silicon Pixels and the Semi-Conductor Tracker (SCT) detectors for $|\eta|<2.5$ and the Transition Radiation Tracker (TRT) covering $|\eta|<2.0$. They provide highresolution coordinate measurements for track reconstruction inside an axial magnetic field of $2 \mathrm{~T}$. A track in the barrel region has typically 3 Pixel hits, 8 SCT hits, and approximately 30 TRT hits.

The material between the interaction point and the MS ranges approximately from 100 to 190 radiation lengths, depending on $\eta$, and consists mostly of calorimeters. The sampling liquid-argon (LAr) electromagnetic calorimeter covers $|\eta|<3.2$ and is surrounded by hadronic calorimeters based on iron and scintillator tiles for $|\eta| \lesssim 1.5$ and on LAr for larger values of $|\eta|$.

Muon identification is performed according to several reconstruction criteria (leading to different muon "types"), according to the available information from the ID, the MS, and the calorimeter sub-detector systems. The different types are:

- Stand-Alone (SA) muons: the muon trajectory is reconstructed only in the MS. The parameters of the muon track at the interaction point are determined by extrapolating the track back to the point of closest approach to the beam line, taking into account the estimated energy loss of the muon in the calorimeters. In general the muon has to tra- verse at least two layers of MS chambers to provide a track measurement. SA muons are mainly used to extend the acceptance to the range $2.5<|\eta|<2.7$ which is not covered by the ID;

- Combined (CB) muon: track reconstruction is performed independently in the ID and MS, and a combined track is formed from the successful combination of a MS track with an ID track. This is the main type of reconstructed muons;

- Segment-tagged (ST) muons: a track in the ID is classified as a muon if, once extrapolated to the MS, it is associated with at least one local track segment in the MDT or CSC chambers. ST muons can be used to increase the acceptance in cases in which the muon crossed only one layer of MS chambers, either because of its low $p_{\mathrm{T}}$ or because it falls in regions with reduced MS acceptance;

- Calorimeter-tagged (CaloTag) muons: a track in the ID is identified as a muon if it could be associated to an energy deposit in the calorimeter compatible with a minimum ionizing particle. This type has the lowest purity of all the muon types but it recovers acceptance in the uninstrumented regions of the MS. The identification criteria of this muon type are optimized for a region of $|\eta|<0.1$ and a momentum range of $25 \lesssim p_{\mathrm{T}} \lesssim 100 \mathrm{GeV}$.

$\mathrm{CB}$ candidates have the highest muon purity. The reconstruction of tracks in the spectrometer, and as a consequence the $\mathrm{SA}$ and $\mathrm{CB}$ muons, is affected by acceptance losses mainly in two regions: at $\eta \approx 0$, where the MS is only partially equipped with muon chambers in order to provide space for the services for the ID and the calorimeters, and in the region $(1.1<\eta<1.3)$ between the barrel and the positive $\eta$ endcap, where there are regions in $\phi$ with only one layer of chambers traversed by muons in the MS, due to the fact that some of the chambers of that region were not yet installed. ${ }^{2}$

The reconstruction of the SA, CB and ST muons (all using the MS information) has been performed using two independent reconstruction software packages, implementing different strategies [10] (named "Chains") both for the reconstruction of muons in the MS and for the ID-MS combination. For the ID-MS combination, the first chain ("Chain 1") performs a statistical combination of the track parameters of the SA and ID muon tracks using the corresponding covariance matrices. The second ("Chain 2") performs a global refit of the muon track using the hits from both the ID and MS sub-detectors. The use of two independent codes provided redundancy and robustness in the ATLAS commissioning phase. A unified reconstruction programme ("Chain 3") has been developed to incorporate the best features of the two chains and has been used, in parallel to the other two, for the reconstruction

\footnotetext{
2 The installation of all the muon chambers in this region has been completed during the 2013-2014 LHC shutdown.
} 
of 2012 data. It is planned to use only Chain 3 for future data taking. So far, the first two chains were used in all ATLAS publications. As the three chains have similar performance, only results for "Chain 1" are shown in the present publication. A summary of the results for the other two chains is reported in Appendix A.

The following quality requirements are applied to the ID tracks used for CB, ST or CaloTag muons:

- at least 1 Pixel hit;

- at least 5 SCT hits;

- at most 2 active Pixel or SCT sensors traversed by the track but without hits;

- in the region of full TRT acceptance, $0.1<|\eta|<1.9$, at least 9 TRT hits.

The number of hits required in the first two points is reduced by one if the track traverses a sensor known to be inefficient according to a time-dependent database. The above requirements are dropped in the region $|\eta|>2.5$, where short ID track segments can be matched to $\mathrm{SA}$ muons to form a $\mathrm{CB}$ muon.

\section{Data and Monte Carlo samples}

\subsection{Data samples}

The results presented in this article are mostly obtained from the analysis of $\sqrt{s}=8 \mathrm{TeV} p p$ collision events corresponding to an integrated luminosity of $20.3 \mathrm{fb}^{-1}$ collected by the ATLAS detector in 2012. Results from $p p$ collisions at $\sqrt{s}=7 \mathrm{TeV}$, collected in 2011, are presented in Appendix B. Events are accepted only if the ID, the MS and the calorimeter detectors were operational and both solenoid and toroid magnet systems were on.

The online event selection was performed by a threelevel trigger system described in Ref. [11]. The performance of the ATLAS muon trigger during the 2012 data taking period is reported in Ref. [12]. The $Z \rightarrow \mu \mu$ candidates have been selected online by requiring at least one muon candidate with $p_{\mathrm{T}}>24 \mathrm{GeV}$, isolated from other activity in the ID. The $J / \psi \rightarrow \mu \mu$ and the $\Upsilon \rightarrow \mu \mu$ samples used for momentum scale and resolution studies have been selected online with two dedicated dimuon triggers that require two opposite-charge muons compatible with the same vertex, with transverse momentum $p_{\mathrm{T}}>6 \mathrm{GeV}$, and the dimuon invariant mass in the range $2.5-4.5 \mathrm{GeV}$ for the $J / \psi$ and $8-11 \mathrm{GeV}$ for the $\Upsilon$ trigger. The $J / \psi \rightarrow \mu \mu$ sample used for the efficiency measurement was instead selected using a mix of single-muon triggers and a dedicated trigger requiring a muon with $p_{\mathrm{T}}>6 \mathrm{GeV}$ and an ID track with $p_{\mathrm{T}}>3.5 \mathrm{GeV}$, such that the invariant mass of the muon+track pair, under a muon mass hypothesis, is in the window 2.7-3.5 GeV. This dedicated trigger operated during the whole data taking period with a prescaled rate of $\approx 1 \mathrm{~Hz}$.

\subsection{Monte Carlo samples}

Monte Carlo samples for the process $p p \rightarrow\left(Z / \gamma^{*}\right) X \rightarrow$ $\mu^{+} \mu^{-} X$, called $Z \rightarrow \mu \mu$ in the following, were generated using POWHEG [13] interfaced to PYTHIA8 [14]. The CT10 [15] parton density functions (PDFs) have been used. The PHOTOS [16] package has been used to simulate final state photon radiation (FSR), using the exponentiated mode that leads to multi-photon emission taking into account $\gamma^{*}$ interference in $Z$ decays. To improve the description of the dimuon invariant mass distribution, the generated lineshape was reweighted using an improved Born approximation with a running-width definition of the $Z$ lineshape parameters. The ALPGEN [17] generator, interfaced with PYTHIA6 [18], was also used to generate alternative $Z \rightarrow \mu \mu$ samples.

Samples of prompt $J / \psi \rightarrow \mu \mu$ and of $\Upsilon \rightarrow \mu \mu$ were generated using PYTHIA8, complemented with PHOTOS to simulate the effects of final state radiation. The samples were generated requiring each muon to have $p_{\mathrm{T}}>6.5(6) \mathrm{GeV}$ for $J / \psi(\Upsilon)$. The $J / \psi$ distribution in rapidity and transverse momentum has been reweighted in the simulated samples to match the distribution observed in the data. The samples used for the simulation of the backgrounds to $Z \rightarrow \mu \mu$ are described in detail in [19], they include $Z \rightarrow \tau \tau, W \rightarrow \mu \nu$ and $W \rightarrow \tau \nu$, generated with POWHEG, $W W, Z Z$ and $W Z$ generated with SHERPA [20], $t \bar{t}$ samples generated with MC@NLO [21] and $b \bar{b}$ as well as $c \bar{c}$ samples generated with PYTHIA6.

All the generated samples were passed through the simulation of the ATLAS detector based on GEANT4 [22,23] and were reconstructed with the same programs used for the data. The ID and the MS were simulated with an ideal geometry without any misalignment. To emulate the effect of the misalignments of the MS chambers in real data, the reconstruction of the muon tracks in the simulated samples was performed using a random set of MS alignment constants. The amount of random smearing applied to these alignment constants was derived from an early assessment of the precision of the alignment, performed with special runs in which the toroidal magnetic field was off. The knowledge of the alignment constants improved with time. In particular the alignment constants used for the reconstruction of the data were more precise than those used to define the random smearing applied in the simulation, resulting in some cases in a worse MS resolution in MC than in data. 


\section{Efficiency}

The availability of two independent detectors to reconstruct the muons (the ID and the MS) enables a precise determination of the muon reconstruction efficiency in the region $|\eta|<2.5$. This is obtained with the so called tag-and-probe method described in the next section. A different methodology, described in Sect. 4.2, is used in the region $2.5<|\eta|<$ 2.7 in which only one detector (the MS) is available.

\subsection{Muon reconstruction efficiency in the region $|\eta|<2.5$}

The tag-and-probe method is employed to measure the reconstruction efficiencies of all muon types within the acceptance of the ID $(|\eta|<2.5)$. The conditional probability that a muon reconstructed by the ID is also reconstructed using the MS as a particular muon type, $P$ (Type $\mid \mathrm{ID})$, with Type $=(\mathrm{CB}, \mathrm{ST})$, can be measured using ID probes. Conversely, the conditional probability that a muon reconstructed by the MS is also reconstructed in the ID, $P$ (ID $\mid \mathrm{MS})$, is measured using MS tracks as probes.

For each muon type, the total reconstruction efficiency is given by:

$\varepsilon($ Type $)=\varepsilon($ Type $\mid$ ID $) \cdot \varepsilon($ ID $)$,

where $\varepsilon$ (ID) is the probability that a muon is reconstructed as an ID track. The quantity $\varepsilon$ (ID) cannot be measured directly and is replaced by $\varepsilon(\mathrm{ID} \mid \mathrm{MS})$ to give the tag-andprobe approximation:

$\varepsilon($ Type $) \simeq \varepsilon($ Type $\mid \mathrm{ID}) \cdot \varepsilon(\mathrm{ID} \mid \mathrm{MS})$.

The level of agreement of the measured efficiency, $\varepsilon^{\text {Data }}$ (Type), with the efficiency measured with the same method in MC, $\varepsilon^{\mathrm{MC}}$ (Type), is expressed as the ratio between these two numbers, called "efficiency scale factor" or SF:

$S F=\frac{\varepsilon^{\text {Data }}(\text { Type })}{\varepsilon^{\mathrm{MC}}(\text { Type })}$.

Possible biases introduced by the tag-and-probe approximation and other systematic effects on the efficiency measurement, which appear both in data and in MC, cancel in the SF. The SF is therefore used to correct the simulation in physics analysis.

\subsubsection{The tag-and-probe method with $Z \rightarrow \mu \mu$ events}

For $Z \rightarrow \mu \mu$ decays, events are selected by requiring two oppositely charged isolated muons ${ }^{3}$ with transverse

\footnotetext{
${ }^{3}$ Here a muon is considered to be isolated when the sum of the momenta of the other tracks with $p_{\mathrm{T}}>1 \mathrm{GeV}$ in a cone of $\Delta R=0.4$ around the muon track is less than 0.15 times the muon momentum itself. Different cone sizes and cuts on the momentum fraction are used in other parts of this paper.
}

momenta of at least $p_{\mathrm{T}}>25$ and $10 \mathrm{GeV}$ respectively and a dimuon invariant mass within $10 \mathrm{GeV}$ of the $Z$-boson mass. The muons are required to be back to back in the transverse plane $(\Delta \phi>2)$. One of the muons is required to be a $\mathrm{CB}$ muon, and to have triggered the readout of the event. This muon is called the "tag". The other muon, the so-called "probe", is required to be a MS track (i.e. a SA or a CB muon) when $\varepsilon(\mathrm{ID} \mid \mathrm{MS})$ is to be measured. The probe is required to be a CaloTag muon for the measurement of $\varepsilon$ (Type|ID). The use of CaloTag muons as the ID probes reduces the background in the $Z \rightarrow \mu \mu$ sample by an order of magnitude without biasing the efficiency measurement. The MS probes are also used to measure the efficiency of CaloTag muons. After selecting all tag-probe pairs, an attempt is made to match the probe to a reconstructed muon: a match is successful when the muon and the probe are close in the $\eta-\phi$ plane $(\Delta R<0.01$ for CaloTag probes to be matched with $\mathrm{CB}$ or ST muons and $\Delta R<0.05$ for MS probes to be matched to ID or CaloTag muons).

\subsubsection{Background treatment in $Z \rightarrow \mu \mu$ events}

Apart from $Z \rightarrow \mu \mu$ events, a small fraction of the selected tag-probe pairs may come from other sources. For a precise efficiency measurement, these backgrounds have to be estimated and subtracted. Contributions from $Z \rightarrow \tau \tau$ and $t \bar{t}$ decays are estimated using MC simulation. Additionally, QCD multijet events and $W \rightarrow \mu \nu$ decays in association with jet activity ( $W+$ jets) can yield tag-probe pairs through secondary muons from heavy- or light-hadron decays. As these backgrounds are approximately charge-symmetric, they are estimated from the data using same-charge (SC) tag-probe pairs. This leads to the following estimate of the oppositecharge (OC) background for each region of the kinematic phase-space:

$N(\mathrm{Bkg})=N_{\mathrm{OC}}^{Z, t \bar{t} \mathrm{MC}}+T \cdot\left(N_{\mathrm{SC}}^{\mathrm{Data}}-N_{\mathrm{SC}}^{Z, t \bar{t} \mathrm{MC}}\right)$

where $N_{\mathrm{OC}}^{Z, t \bar{t} \mathrm{MC}}$ is the contribution from $Z \rightarrow \tau \tau$ and $t \bar{t}$ decays, $N_{\mathrm{SC}}^{\mathrm{Data}}$ is the number of SC pairs measured in data and $N_{\mathrm{SC}}^{Z, t \bar{t} \mathrm{MC}}$ is the estimated contribution of the $Z \rightarrow \mu \mu, Z \rightarrow$ $\tau \tau$ and $t \bar{t}$ processes to the SC sample. $T$ is a global transfer factor that takes into account the residual charge asymmetry of the QCD multijet and W+jets samples, estimated using the simulation:

$T=1+\theta ; \quad \theta=\frac{N_{\mathrm{OC}}^{\mathrm{QCD}+\mathrm{W} M C}-N_{\mathrm{SC}}^{\mathrm{QCD}+\mathrm{W} M C}}{N_{\mathrm{SC}}^{\mathrm{Data}}}$.

For the kinematic region covered by the measurement, the transfer factor is $T=1.15$ for CaloTag probes. For the MS probes the misidentification rate is low and the residual QCD 
multijet background has a large contribution from oppositely charged muon pairs in $b \bar{b}$ decays, leading to $T=2.6$. The efficiency for finding a muon of type A given a probe of type B, corrected for the effect of background, can then be computed as:

$\varepsilon(\mathrm{A} \mid \mathrm{B})=\frac{N_{\text {Probes }}^{\mathrm{Match}}(\text { Data })-N_{\text {Probes }}^{\mathrm{Match}}(\mathrm{Bkg})}{N_{\text {Probes }}^{\mathrm{Al}}(\text { Data })-N_{\text {Probes }}^{\mathrm{All}}(\mathrm{Bkg})}$,

where $N_{\text {Probes }}^{\text {All }}$ stands for the total number of probes considered and $N_{\text {Probes }}^{\text {Match }}$ is the number of probes successfully matched to a reconstructed muon of type A. According to the background estimate reported above, the sample of selected CaloTag probes is more than $99.5 \%$ pure in $Z \rightarrow \mu \mu$ decays, as shown in Fig. 1. The $Z \rightarrow \mu \mu$ purity is maximal for muon $p_{\mathrm{T}} \simeq 40 \mathrm{GeV}$ and decreases to $98.5 \%$ (97\%) for $p_{\mathrm{T}}=10(100) \mathrm{GeV}$. The $Z \rightarrow \mu \mu$ purity has a weak dependence on the average number of inelastic $p p$ interactions per bunch crossing, $\langle\mu\rangle$, decreasing from $99.8 \%$ at $\langle\mu\rangle=10$ to $99.5 \%$ at $\langle\mu\rangle=34$. A purity above $99.8 \%$ is obtained in the selection of MS probes, with weaker dependence on $p_{\mathrm{T}}$ and $\langle\mu\rangle$.

\subsubsection{Low $p_{\mathrm{T}}$ efficiencies from $J / \psi \rightarrow \mu \mu$ decays}

The efficiencies extracted from $Z \rightarrow \mu \mu$ decays are complemented at low $p_{\mathrm{T}}$ with results derived from a sample of $J / \psi \rightarrow \mu \mu$ events. In 2012 ATLAS collected approximately $2 \mathrm{M} J / \psi \rightarrow \mu \mu$ decays which were not biased by dimuon triggers requirements, using a combination of single muon triggers (isolated and non-isolated) and the dedicated "muon + track" trigger described in Sect. 3.1.

The analysis proceeds in a similar manner to the $Z \rightarrow \mu \mu$ with some modifications due to the different kinematics of the $J / \psi$. Tags are required to be CB muons with $p_{\mathrm{T}}>4 \mathrm{GeV}$ and $|\eta|<2.5$. As with the $Z$, the tag must have triggered the read-out of the event. Probes are sought from amongst the ID tracks and must have $p_{\mathrm{T}}>2.5 \mathrm{GeV}$ and $|\eta|<2.5$, opposite charge to the tag muon, and must form with the tag an invariant mass in the window $2.7-3.5 \mathrm{GeV}$. Finally the tag-probe pairs must fit to a common vertex with a very loose quality cut of $\chi^{2}<200$ for one degree of freedom, which removes tracks from different vertices, without any significant efficiency loss. Muon reconstruction efficiencies are then derived by binning in small cells of $p_{\mathrm{T}}$ and $\eta$ of the probe tracks. Invariant mass distributions are built in each cell for two samples: (a) all tag-probe pairs and (b) tag-probe pairs in which the probe failed to be reconstructed in the MS. The invariant mass distributions are fitted with a signal plus background model to obtain the number of $J / \psi$ signal events in the two samples, called $N_{a}\left(p_{\mathrm{T}}, \eta\right)$ and $N_{b}\left(p_{\mathrm{T}}, \eta\right)$, respectively. The fit model is a Gaussian plus a second order polynomial for the background. The two samples are fitted simul-
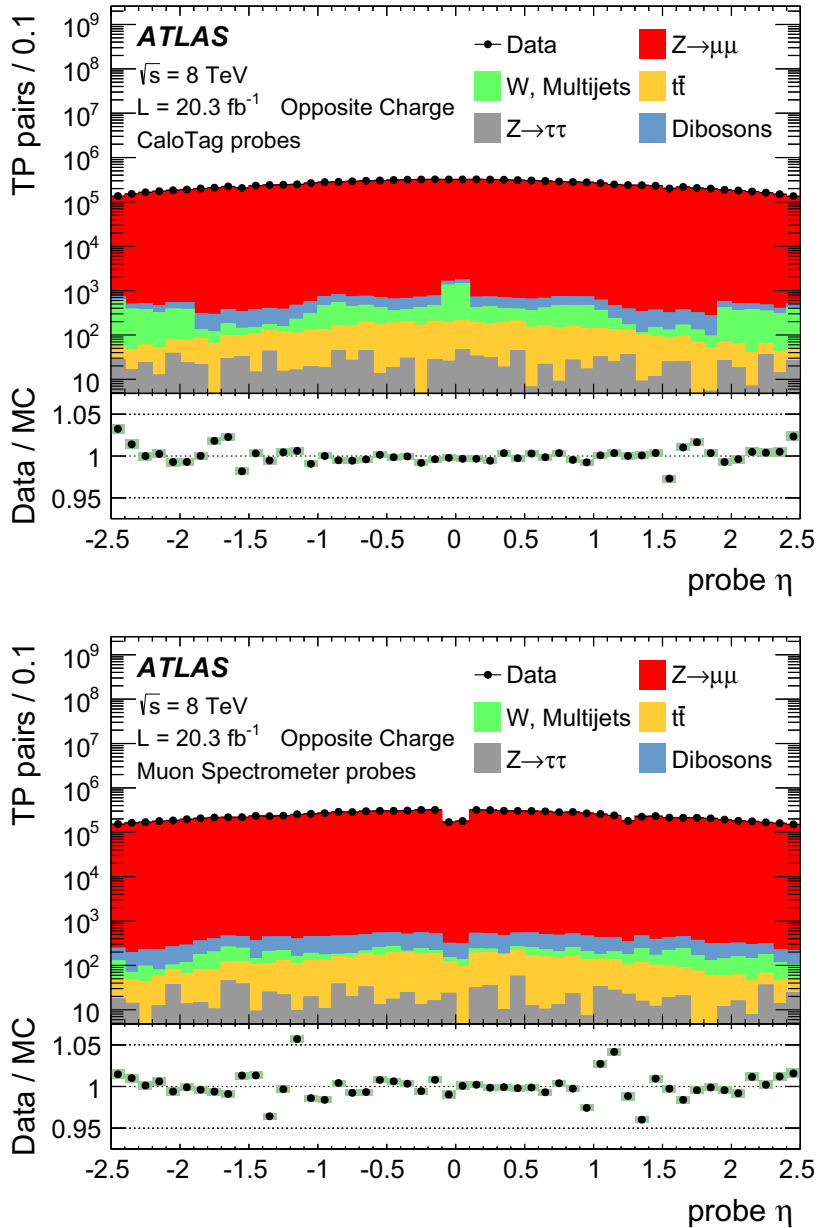

Fig. 1 Pseudorapidity distribution of the CaloTag (top) or MS (bottom) probes used in the tag-and-probe analysis. The bottom panel shows the ratio between observed and expected counts. The sum of the MC samples is normalized to the number of events in the data. The green band represents the statistical uncertainty

taneously using the same mean and width to describe the signal. The MS reconstruction efficiency in a given $\left(p_{\mathrm{T}}, \eta\right)$ cell is then defined as:

$\varepsilon_{p_{\mathrm{T}}, \eta}($ Type $\mid \mathrm{ID})=1-\frac{N_{b}\left(p_{\mathrm{T}}, \eta\right)}{N_{a}\left(p_{\mathrm{T}}, \eta\right)}$.

The largest contribution to the systematic uncertainty originates from the model used in the fit. This uncertainty was estimated by changing the background model to a first or a third order polynomial and by relaxing the constraint that the mass and the width of the $J / \psi$ signal are the same between the two samples. The resulting variations in the efficiency are added in quadrature to the statistical uncertainty to give the total uncertainty on the efficiency. The efficiency integrated over the full $\eta$ region is obtained as an average of the efficiencies of the different $\eta$ cells. This method ensures a reduced dependency on local variations of background 
and resolution, and on the kinematic distribution of the probes.

\subsubsection{Systematic uncertainties}

The main contributions to the systematic uncertainty on the measurement of the efficiency SFs are shown in Fig. 2, as a function of $\eta$ and $p_{\mathrm{T}}$, and are discussed below (the labels in parenthesis refer to the legend of Fig. 2):

- (Bkg) the uncertainty on the data-driven background estimate is evaluated by varying the charge-asymmetry parameter $\theta$ of Eq. (5) by $\pm 100 \%$. This results in an uncertainty of the efficiency measurement below $0.1 \%$ in a large momentum range, reaching up to $0.2 \%$ for low muon momenta where the contribution of the background is most significant.

- (dR) the choice of the cone size used for matching reconstructed muons to probe objects has been optimized to minimize the amount of matches with wrong tracks while keeping the maximum match efficiency for correct tracks. A systematic uncertainty is evaluated by varying the cone size by $\pm 50 \%$. This yields an uncertainty of $\approx 0.1 \%$.

- (TP approximation) possible biases in the tag-and-probe method, for example due to different distributions between MS probes and "true" muons or due to correlation between ID and MS efficiencies, are investigated. The simulation is used to compare the efficiency measured with the tag-andprobe method with the "true" MC efficiency calculated as the fraction of generator-level muons that are successfully reconstructed. Agreement within less than $0.1 \%$ is observed, with the exception of the region $|\eta|<0.1$. In the extraction of the data/MC scale factors, the difference between the measured and the "true" efficiency cancels to first order. To take into account possible imperfection of the simulation, half the observed difference is used as an additional systematic uncertainty on the SF.

- (Probes) the scale factor maps may be sensitive to disagreements between data and simulation in the kinematic distributions of the probes. The corresponding systematic uncertainty is estimated by reweighting the distribution of the probes in the simulation to bring it into agreement with the data. The resulting effect on the efficiency is below $0.1 \%$ over most of the phase space.

- $\left(\right.$ Low $p_{\mathrm{T}}$ ) for $4<p_{\mathrm{T}}<10 \mathrm{GeV}$ the systematic uncertainties are obtained from the analysis performed with the $J / \psi \rightarrow \mu \mu$ sample, as discussed in Sect. 4.1.3 (not shown in Fig. 2). The resulting uncertainty on the low- $p_{\mathrm{T}} \mathrm{SFs}$ ranges between $0.5 \%$ and $2 \%$, depending on $p_{\mathrm{T}}$ and $\eta$ and is dominated by the uncertainty on the background model.

- (High $p_{\mathrm{T}}$ ) no significant dependence of the measured SFs with $p_{\mathrm{T}}$ was observed in the momentum range considered.
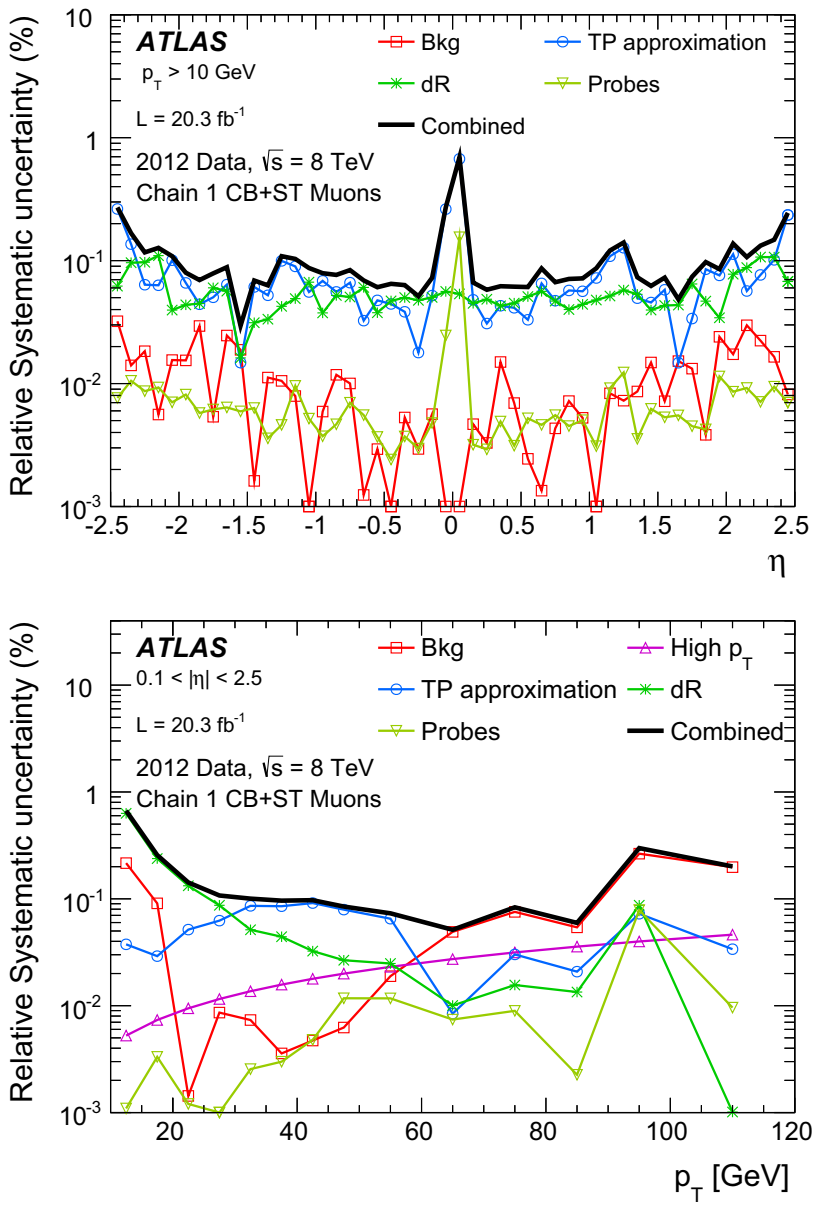

Fig. 2 Systematic uncertainty on the efficiency scale factor for $\mathrm{CB}+\mathrm{ST}$ muons, obtained from $Z \rightarrow \mu \mu$ data, as a function of $\eta$ (top) and $p_{\mathrm{T}}$ (bottom) for muons with $p_{\mathrm{T}}>10 \mathrm{GeV}$. The background systematic uncertainty in the last two bins of the bottom plot is affected by a large statistical uncertainty. The combined systematic uncertainty is the sum in quadrature of the individual contributions

An upper limit on the SF variation for large muon momenta has been extracted by using a MC simulation with built-in imperfections, including a realistic residual misalignment of the detector components or a $10 \%$ variation of the muon energy loss. On the basis of this, a systematic uncertainty of $\pm 0.42 \% \times\left(p_{\mathrm{T}} / 1 \mathrm{TeV}\right)$ is obtained.

\subsubsection{Results}

Figure 3 shows the muon reconstruction efficiency $\varepsilon$ (Type) as a function of $\eta$ as measured from $Z \rightarrow \mu \mu$ events. The combination of all the muon reconstruction types (for $\mathrm{CB}, \mathrm{ST}$, and CaloTag muons) gives a uniform muon reconstruction efficiency of about $99 \%$ over most the detector regions. The use of ST muons allows the recovery of efficiency especially in the region $1.1<\eta<1.3$ (from $85 \%$ to $99 \%$ ) in which 


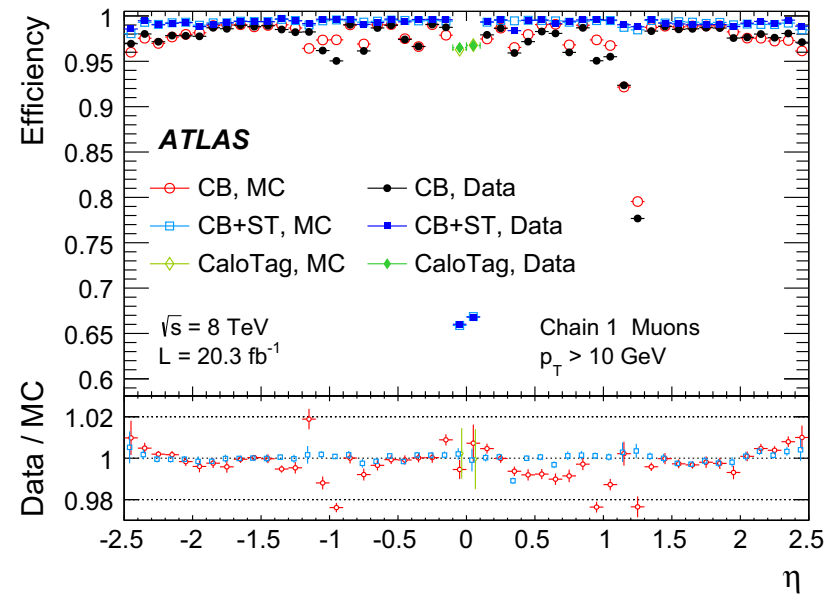

Fig. 3 Muon reconstruction efficiency as a function of $\eta$ measured in $Z \rightarrow \mu \mu$ events for muons with $p_{\mathrm{T}}>10 \mathrm{GeV}$ and different muon reconstruction types. CaloTag muons are only shown in the region $|\eta|<$ 0.1 , where they are used in physics analyses. The error bars on the efficiencies indicate the statistical uncertainty. The panel at the bottom shows the ratio between the measured and predicted efficiencies. The error bars on the ratios are the combination of statistical and systematic uncertainties

part of the MS chambers were not installed, as discussed in Sect. 2. The remaining inefficiency of the combination of $\mathrm{CB}$ or ST muons $(\mathrm{CB}+\mathrm{ST})$ at $|\eta|<0.1(66 \%)$ is almost fully recovered by the use of CaloTag muons (97\%).

The efficiencies measured in experimental and simulated data are in good agreement, in general well within $1 \%$. The largest differences are observed in the CB muons. To reconstruct an MS track, the Chain 1 reconstruction requires track segments in at least two layers of precision chambers (MDT or CSC) and at least one measurement of the $\phi$ coordinate from trigger chambers (RPC or TGC). These requirements introduce some dependency on detector conditions and on the details of the simulation in the regions in which only two layers of precision chambers or only one layer of trigger chambers are crossed by the muons. This results in a reduction of efficiency in data with respect to MC of approximately $1 \%$ in the region of $\eta \sim 0.5$ due the RPC detector conditions and to local deviations up to about $2 \%$ at $0.9<|\eta|<1.3$ related to imperfections in the simulation of the barrel-endcap transition region. For the $\mathrm{CB}+\mathrm{ST}$ muons the agreement between data and $\mathrm{MC}$ is very good, with the only exception of a lowefficiency region in data at $\eta=0.3-0.4$ related to an inactive portion of an MDT chamber (not included in MC) in a region with reduced coverage due to the supporting structure of the ATLAS detector. ${ }^{4}$

The ID muon reconstruction efficiency, $\varepsilon(\mathrm{ID} \mid \mathrm{MS})$, for $p_{\mathrm{T}}>10 \mathrm{GeV}$ as a function of $\eta$ and $p_{T}$ is shown in Fig. 4. The efficiency is greater than 0.99 and there is very good agree-

\footnotetext{
4 This effect is also visible in Fig. 9 at $\phi \simeq-1$.
}
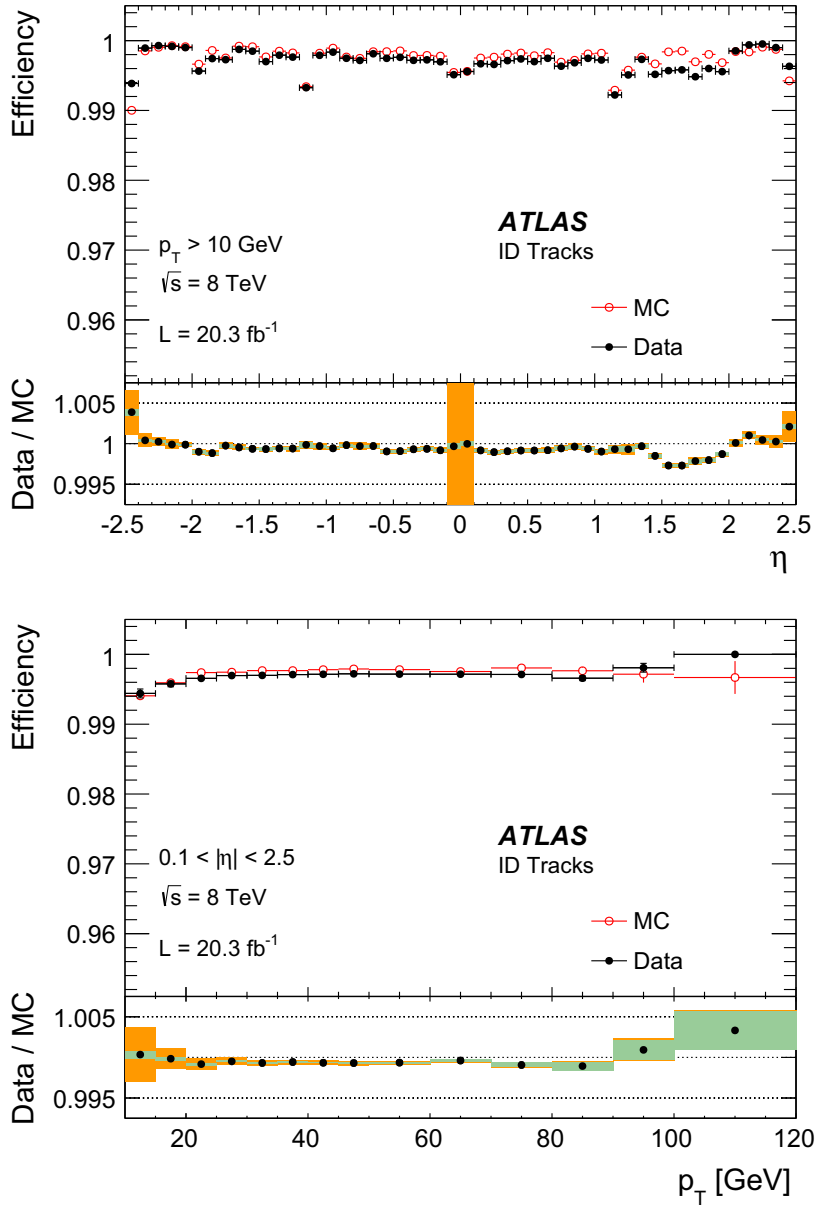

Fig. 4 ID muon reconstruction efficiency as a function of $\eta$ (top) and $p_{\mathrm{T}}$ (bottom) measured in $Z \rightarrow \mu \mu$ events for muons with $p_{\mathrm{T}}>10 \mathrm{GeV}$. The error bars on the efficiencies indicate the statistical uncertainty. The panel at the bottom shows the ratio between the measured and predicted efficiencies. The green areas depict the pure statistical uncertainty, while the orange areas also include systematic uncertainties

ment between data and MC. The small efficiency reduction in the region $1.5<\eta<2$ is related to temporary hardware problems in the silicon detectors. The larger uncertainty at $|\eta|<0.1$ is related to the limited MS coverage in that region.

Figure 5 shows the reconstruction efficiencies for $\mathrm{CB}$ and for $\mathrm{CB}+\mathrm{ST}$ muons as a function of the transverse momentum, including results from $Z \rightarrow \mu \mu$ and $J / \psi \rightarrow \mu \mu$. A steep increase of the efficiency is observed at low $p_{\mathrm{T}}$, in particular for the CB reconstruction, since a minimum momentum of approximately $3 \mathrm{GeV}$ is required for a muon to traverse the calorimeter material and cross at least two layers of MS stations before being bent back by the magnetic field. Above $p_{\mathrm{T}} \approx 20 \mathrm{GeV}$, the reconstruction efficiency for both $\mathrm{CB}$ and $\mathrm{CB}+\mathrm{ST}$ muons is expected to be independent of the transverse momentum. This is confirmed within $0.5 \%$ by the $Z \rightarrow \mu \mu$ data. The drop in efficiency observed in the $J / \psi$ data at $p_{\mathrm{T}}>15 \mathrm{GeV}$ is due to the inefficiency of the MS 

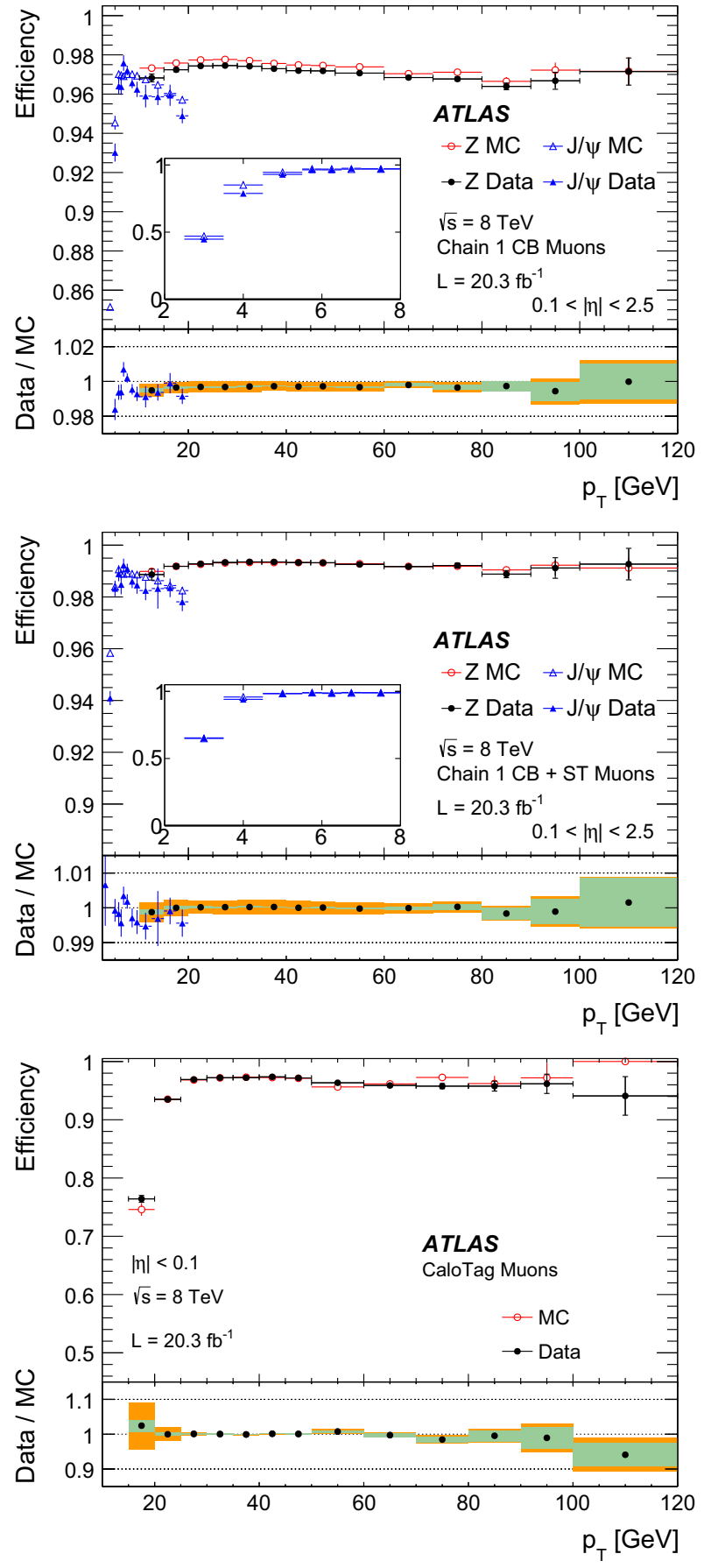

Fig. 5 Reconstruction efficiency for $\mathrm{CB}$ (top), $\mathrm{CB}+\mathrm{ST}$ (middle) and CaloTag (bottom) muons as a function of the $p_{\mathrm{T}}$ of the muon, for muons with $0.1<|\eta|<2.5$ for $\mathrm{CB}$ and $\mathrm{CB}+\mathrm{ST}$ muons and for $|\eta|<0.1$ for CaloTag muons. The upper two plots also show the result obtained with $Z \rightarrow \mu \mu$ and $J / \psi \rightarrow \mu \mu$ events. The insets on the upper plots show the detail of the efficiency as a function of $p_{\mathrm{T}}$ in the low $p_{\mathrm{T}}$ region. The CaloTag muon efficiency (bottom) is only measured with $Z \rightarrow$ $\mu \mu$ events. The error bars on the efficiencies indicate the statistical uncertainty for $Z \rightarrow \mu \mu$ and include also the fit model uncertainty for $J / \psi \rightarrow \mu \mu$. The panel at the bottom shows the ratio between the measured and predicted efficiencies. The green areas show the pure statistical uncertainty, while the orange areas also include systematic uncertainties

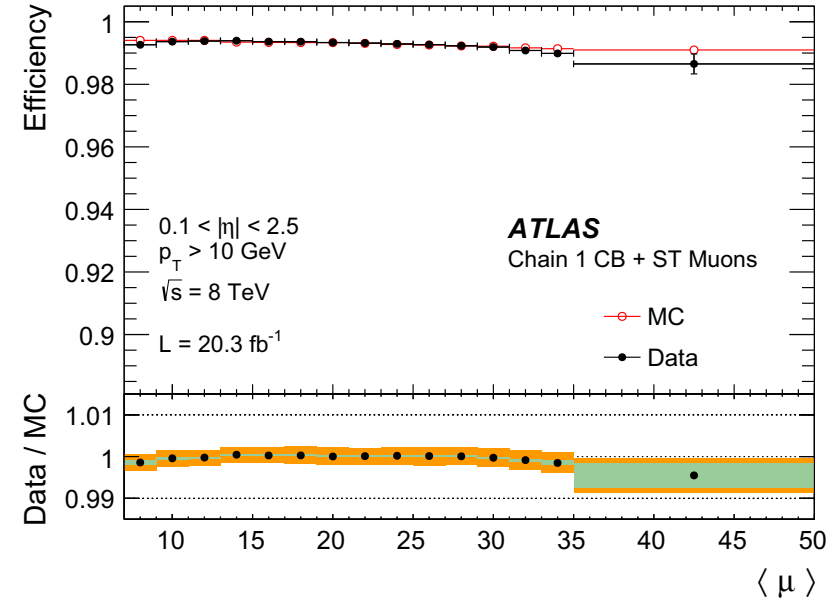

Fig. 6 Measured $\mathrm{CB}+\mathrm{ST}$ muon reconstruction efficiency for muons with $p_{\mathrm{T}}>10 \mathrm{GeV}$ as a function of the average number of inelastic $p p$ collisions per bunch crossing $\langle\mu\rangle$. The error bars on the efficiencies indicate the statistical uncertainty. The panel at the bottom shows the ratio between the measured and predicted efficiencies. The green areas depict the pure statistical uncertainty, while the orange areas also include systematic uncertainties

reconstruction for muon pairs with small angular separation as in the case of highly boosted $J / \psi$. This effect is well reproduced by MC and the SF of the $J / \psi \rightarrow \mu \mu$ analysis are in good agreement with those from $Z \rightarrow \mu \mu$ in the overlap region. The CaloTag muon efficiency reaches a plateau of approximately 0.97 above $p_{\mathrm{T}} \gtrsim 30 \mathrm{GeV}$, where it is well predicted by the MC.

Figure 6 shows the reconstruction efficiency for $\mathrm{CB}+\mathrm{ST}$ muons as a function of $\langle\mu\rangle$, showing a high value (on average above 0.99 ) and remarkable stability. A small efficiency drop of about $1 \%$ is only observed for $\langle\mu\rangle \gtrsim 35$. This is mainly caused by limitations of the MDT readout electronics in the high-rate regions close to the beam lines. These limitations are being addressed in view of the next LHC run.

\subsection{Muon reconstruction efficiency for $|\eta|>2.5$}

As described in the previous sections, the $\mathrm{CB}$ muon reconstruction is limited by the ID acceptance which covers the pseudo-rapidity region $|\eta|<2.5$. Above $|\eta|=2.5$, SA muons are the only muon type that provides large efficiency. A measurement of the efficiency SF for muons in the range $2.5<|\eta|<2.7$, hereafter called high- $\eta$, is needed for the physics analyses that exploit the full MS acceptance.

A comparison with the Standard Model calculations for $Z \rightarrow \mu \mu$ events is used to measure the reconstruction efficiency SF in the high- $\eta$ region. To reduce the theoretical and experimental uncertainties, the efficiency SF is calculated from the double ratio 


$$
\mathrm{SF}=\frac{\frac{N^{\text {Data }}\left(2.5<\left|\eta_{\mathrm{fwd}}\right|<2.7\right)}{N^{\mathrm{MC}}\left(2.5<\left|\eta_{\mathrm{fwd}}\right|<2.7\right)}}{\frac{N^{\text {Data }}\left(2.2<\left|\eta_{\mathrm{fwd}}\right|<2.5\right)}{N^{\mathrm{MC}}\left(2.2<\left|\eta_{\mathrm{fwd}}\right|<2.5\right)}}
$$

where the numerator is the ratio of the number of $Z \rightarrow \mu \mu$ candidates in data and in MC for which one of the muons, called the forward muon, is required to be in the high- $\eta$ region $2.5<\left|\eta_{\text {fwd }}\right|<2.7$ while the other muon from the $Z$ decay, called the central muon, is required to have $|\eta|<2.5$. The denominator is the ratio of $Z \rightarrow \mu \mu$ candidates in data over $\mathrm{MC}$ with the forward muon lying in the control region $2.2<$ $\left|\eta_{\mathrm{fwd}}\right|<2.5$ and the central muon in the region $|\eta|<2.2$. In both the numerator and denominator the central muon is required to be a $\mathrm{CB}$ muon while the forward muon can either be a $\mathrm{CB}$ or SA muon. The simulation of muons with $|\eta|<2.5$ is corrected using the standard SF described in the previous section.

The selection of the central muon is similar to that of the tag muon in the tag-and-probe method. It is required to have triggered the event readout, to be isolated and to have transverse momentum $p_{\mathrm{T}}>25 \mathrm{GeV}$. The requirements for the forward muon include calorimeter-based isolation, requiring the transverse energy $E_{T}$ measured in the calorimeter in a cone of $\Delta R=0.2$ (excluding the energy lost by the muon itself) around the muon track, to be less than $10 \%$ of the muon $p_{\mathrm{T}}$. The central and forward muons are required to have opposite charge, a dimuon invariant mass within $10 \mathrm{GeV}$ of the $Z$ mass, and a separation in $(\eta, \phi)$ space of $\Delta R>0.2$.

Different sources of systematic uncertainties have been considered: a first group is obtained by varying the $p_{\mathrm{T}}$ and isolation cuts on the central muons and the dimuon mass window. These variations produce effects of less than $0.3 \%$ in the efficiency SF for the $p_{\mathrm{T}}$ range $20-60 \mathrm{GeV}$. The effect of the calorimetric isolation on the efficiency SF yields an uncertainty of less than $1 \%$, which is estimated by comparing the nominal SF values with the ones extracted when no calorimetric isolation is applied on the forward muons and by studying the dependence of this cut on the number of $p p$ interactions. The contribution from the background processes, mainly dimuons from $b$ and $\bar{b}$ decays, has been studied using MC background samples and found to be negligible.

The theoretical uncertainty from higher-order corrections is estimated by varying the renormalization and factorization scales in the POWHEG NLO calculation at the generator level and is found to produce a negligible effect on the ratio of Eq. (8). The uncertainty from the knowledge of the parton densities is estimated by reweighting the PDFs used in the MC samples from CT10 to MSTW2008NLO [24] and by studying, at the generator level, the effect of the uncertainty associated to the MSTW2008 PDF set on the double ratio of Eq. (8), obtaining an overall theoretical uncertainty of less than $0.55 \%$.

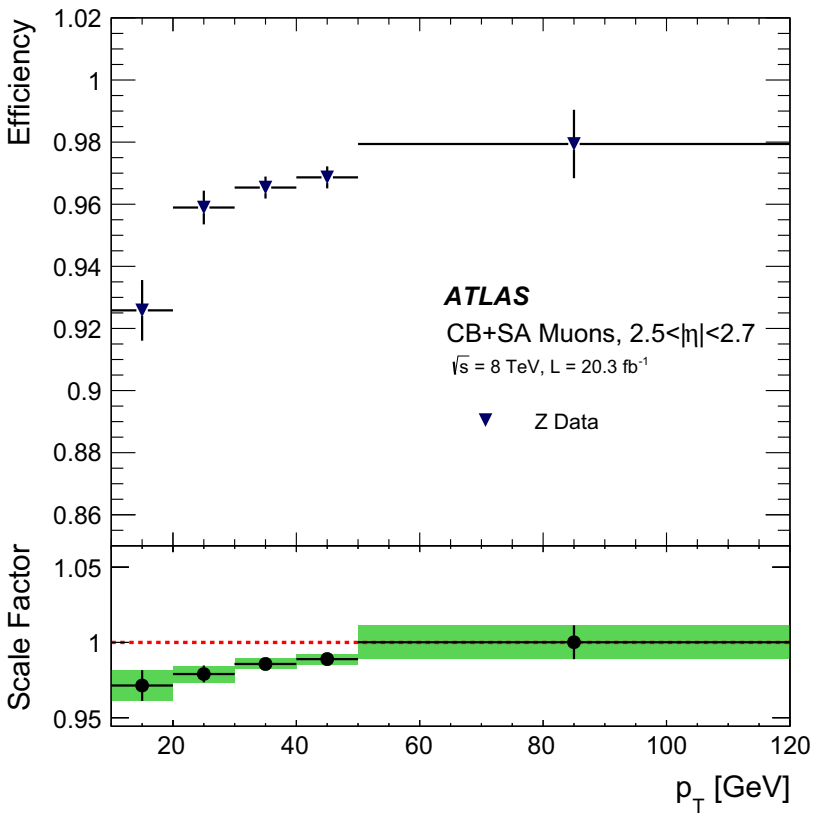

Fig. 7 Reconstruction efficiency for muons within $2.5<|\eta|<2.7$ from $Z \rightarrow \mu \mu$ events. The upper plot shows the efficiency obtained as the product of scale factor (Eq. 8) and the MC efficiency. The lower plot shows the scale factor. The error bars correspond to the statistical uncertainty while the green shaded band corresponds to the statistical and systematic uncertainty added in quadrature

The efficiency in this region is obtained as the product of the SF and the "true" MC efficiency, calculated as the fraction of generator-level muons that are successfully reconstructed. The reconstruction efficiency and the SF for muons in the high- $\eta$ region is shown in Fig. 7 as a function of the muon $p_{\mathrm{T}}$.

\subsection{Scale factor maps}

The standard approach used in ATLAS for physics analysis is to correct the muon reconstruction efficiency in the simulation using efficiency scale factors (SFs). The SFs are obtained with the tag-and-probe method using $Z \rightarrow \mu \mu$ events, as described above, and are provided to the analyses in the form of $\eta-\phi$ maps. Since no significant $p_{\mathrm{T}}$ dependence of the SF has been observed, no $p_{\mathrm{T}}$ binning is used in the SF maps. Different maps are produced for different data taking sub-periods with homogeneous detector conditions. The whole 2012 dataset is divided into 10 sub-periods. For each analysis, the final map is obtained as an average of the maps for all sub-periods, weighted by the periods' contribution to the integrated luminosity under study.

Figures 8 and 9 show the maps of the efficiencies measured using the data in the $\eta-\phi$ plane and the corresponding Scale Factors. The large data sample allows for a precise resolution of localized efficiency losses, for example in the 

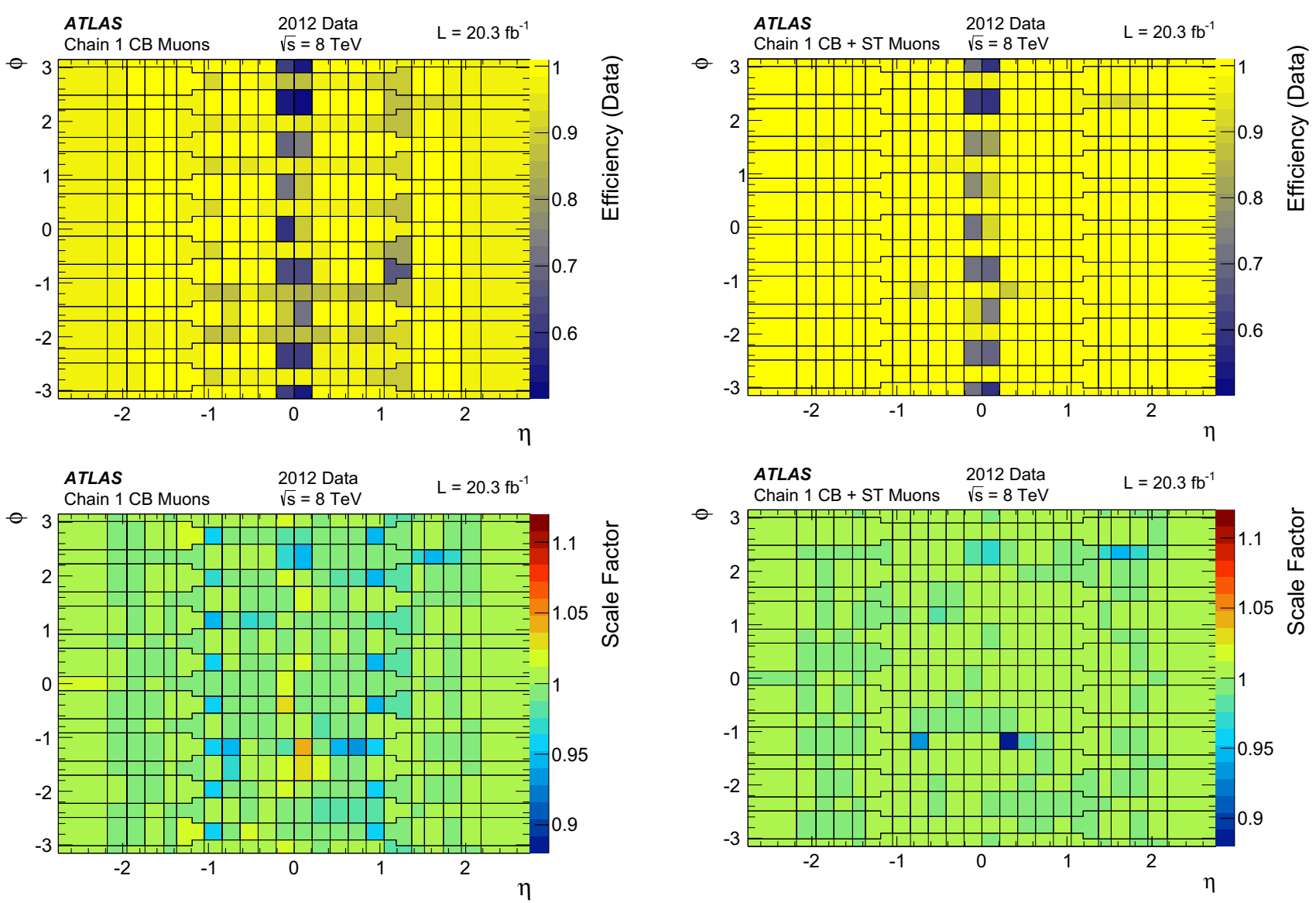

Fig. 8 Reconstruction efficiency measured in the experimental data (top), and the data/MC efficiency scale factor (bottom) for $\mathrm{CB}$ muons as a function of $\eta$ and $\phi$ for muons with $p_{\mathrm{T}}>10 \mathrm{GeV}$

muon spectrometer for $|\eta| \sim 0$ due to limited coverage. The SF maps show local differences between data and MC related to detector conditions as discussed in Sect. 4.1.5.

\section{Momentum scale and resolution}

The large samples of $J / \psi \rightarrow \mu \mu, \Upsilon \rightarrow \mu \mu$ and $Z \rightarrow \mu \mu$ decays collected by ATLAS are used to study in detail the muon momentum scale and resolution. The ATLAS simulation includes the best knowledge of the detector geometry, material distribution, and physics model of the muon interaction at the time of the MC events were generated. Additional corrections are needed to reproduce the muon momentum resolution and scale of experimental data at the level of precision that can be obtained using high-statistics samples of dimuon resonances. Section 5.1 describes the methodology used to extract the corrections to be applied to the MC simulation. In Sect. 5.2, the muon momentum scale and resolution is studied in the data and in MC samples with and without corrections.
Fig. 9 Reconstruction efficiency measured in the experimental data (top) and the data/MC efficiency scale factor (bottom) for $\mathrm{CB}+\mathrm{ST}$ muons as a function of $\eta$ and $\phi$ for muons with $p_{\mathrm{T}}>10 \mathrm{GeV}$

\subsection{Corrections to the muon momentum in MC}

Similarly to Ref. [9], the simulated muon transverse momenta reconstructed in the ID and in the MS sub-detectors, $p_{\mathrm{T}}^{\mathrm{MC}, \mathrm{Det}}$, where Det $=\mathrm{ID}, \mathrm{MS}$, are corrected using the following equation:

$$
\begin{aligned}
& p_{\mathrm{T}}^{\text {Cor,Det }}=\frac{p_{\mathrm{T}}^{\mathrm{MC}, \text { Det }}+\sum_{n=0}^{1} s_{n}^{\text {Det }}(\eta, \phi)\left(p_{\mathrm{T}}^{\mathrm{MC}, \text { Det }}\right)^{n}}{1+\sum_{m=0}^{2} \Delta r_{m}^{\text {Det }}(\eta, \phi)\left(p_{\mathrm{T}}^{\mathrm{MC}, \text { Det }}\right)^{m-1} g_{m}} \\
& \text { (with } s_{0}^{\mathrm{ID}}=0 \text { and } \Delta r_{0}^{\mathrm{ID}}=0 \text { ), }
\end{aligned}
$$

where $g_{m}$ are normally distributed random variables with mean 0 and width 1 and the terms $\Delta r_{m}^{\text {Det }}(\eta, \phi)$ and $s_{n}^{\text {Det }}(\eta, \phi)$ describe, respectively, the momentum resolution smearing and the scale corrections applied in a specific $\eta, \phi$ detector region. The motivations for Eq. (9) are the following:

- corrections are defined in $\eta-\phi$ detector regions such that in each region the variation of momentum resolution and scale, and therefore of their possible corrections, are 
expected to be small. In particular the nominal muon identification acceptance region (up to $|\eta|=2.7$ ) is divided in $18 \eta$ sectors of size $\Delta \eta$ between 0.2 and 0.4 , for both the MS and the ID. In addition, the MS is divided into two types of $\phi$ sectors of approximate size of $\pi / 8$, exploiting the octagonal symmetry of the magnetic system : the sectors that include the magnet coils (called "small sectors") and the sectors between two coils (called "large sectors").

- The $\Delta r_{m}^{\text {Det }}(\eta, \phi)$ correction terms introduce a $p_{\mathrm{T}}$ dependent momentum smearing that effectively increases the relative momentum resolution, $\frac{\sigma\left(p_{\mathrm{T}}\right)}{p_{\mathrm{T}}}$, when underestimated by the simulation. The $\Delta r_{m}^{\text {Det }}(\eta, \phi)$ terms can be related to different sources of experimental resolution by comparing the coefficient of the $p_{\mathrm{T}}$ powers in the denominator of Eq. (9) to the following empirical parametrization of the muon momentum resolution (see for example [25]):

$$
\frac{\sigma\left(p_{\mathrm{T}}\right)}{p_{\mathrm{T}}}=r_{0} / p_{\mathrm{T}} \oplus r_{1} \oplus r_{2} \cdot p_{\mathrm{T}},
$$

where $\oplus$ denotes a sum in quadrature. The first term (proportional to $1 / p_{\mathrm{T}}$ ) accounts for fluctuations of the energy loss in the traversed material. Multiple scattering, local magnetic field inhomogeneities and local radial displacements are responsible for the second term (constant in $p_{\mathrm{T}}$ ). The third term (proportional to $p_{\mathrm{T}}$ ) describes intrinsic resolution effects caused by the spatial resolution of the hit measurements and by residual misalignment. Energy loss fluctuations are relevant for muons traversing the calorimeter in front of the MS but they are negligible in the ID measurement. For this reason $\Delta r_{0}^{\mathrm{ID}}$ is set to zero in Eq. (9).

- Imperfect knowledge of the magnetic field integral and of the radial dimension of the detector are reflected in the multiplicative momentum scale difference $s_{1}^{\text {Det }}$ between data and simulation. In addition, the $s_{0}^{\mathrm{MS}}(\eta, \phi)$ term is necessary to model the $p_{\mathrm{T}}$ scale dependence observed in the MS momentum reconstruction due to differences between data and MC in the energy loss of muons passing through the calorimeter and other materials between the interaction point and the MS. As the energy loss between the interaction point and the ID is negligible, $s_{0}^{\mathrm{ID}}(\eta)$ is set to zero.

The separate correction of ID and MS momentum reconstruction allows a direct understanding of the sources of the corrections. In a second step the corrections are propagated to the $\mathrm{CB}$ momentum reconstruction, $p_{\mathrm{T}}^{\mathrm{Cor}, \mathrm{CB}}$, using a weighted average:

$$
p_{\mathrm{T}}^{\mathrm{Cor}, \mathrm{CB}}=f \cdot p_{\mathrm{T}}^{\mathrm{Cor}, \mathrm{ID}}+(1-f) \cdot p_{\mathrm{T}}^{\mathrm{Cor}, \mathrm{MS}},
$$

with the weight $f$ derived for each muon by expressing the $\mathrm{CB}$ transverse momentum before corrections, $p_{\mathrm{T}}^{\mathrm{MC}, \mathrm{CB}}$, as a linear combination of $p_{\mathrm{T}}^{\mathrm{MC}, \mathrm{ID}}$ and $p_{\mathrm{T}}^{\mathrm{MC}, \mathrm{MS}}$ :

$p_{\mathrm{T}}^{\mathrm{MC}, \mathrm{CB}}=f \cdot p_{\mathrm{T}}^{\mathrm{MC}, \mathrm{ID}}+(1-f) \cdot p_{\mathrm{T}}^{\mathrm{MC}, \mathrm{MS}}$

and solving the corresponding linear equation.

\subsubsection{Correction extraction using a template fit to $J / \psi \rightarrow \mu \mu$ and $Z \rightarrow \mu \mu$ events}

The MS and ID correction parameters contained in Eq. (9) need to be extracted from data. For this purpose, a MC template maximum likelihood fit is used to compare the simulation to the data for $J / \psi \rightarrow \mu \mu$ and $Z \rightarrow \mu \mu$ candidate events: this gives sensitivity to reconstructed muon momenta in the $p_{\mathrm{T}}$ range from a few $\mathrm{GeV}$ to $\approx 100 \mathrm{GeV}$. The dataset used for the correction extraction consists of $6 \mathrm{M} \mathrm{J} / \psi \rightarrow \mu \mu$ and $9 \mathrm{M} Z \rightarrow \mu \mu$ candidates passing the final selection.

The $J / \psi \rightarrow \mu \mu$ and $Z \rightarrow \mu \mu$ candidates have been selected online according to the requirements described in Sect. 3.1 and, offline, by requiring two CB muons. For the correction extraction in a specific $\eta-\phi$ Region Of Fit (ROF), the ID and MS reconstructed momenta are considered individually. All the events with at least one of the two muons in the ROF contribute to the correction extraction fit. The angles from the $\mathrm{CB}$ reconstruction are used to define the ROF and to calculate the invariant mass distributions.

The ID corrections are extracted using the distribution of the ID dimuon invariant mass, $m_{\mu \mu}^{\mathrm{ID}}$. Events with $m_{\mu \mu}^{\mathrm{ID}}$ in the window $2.76-3.6 \mathrm{GeV}$ and $p_{\mathrm{T}}^{\mathrm{ID}}$ in the range $8-17 \mathrm{GeV}$ are selected as $J / \psi \rightarrow \mu \mu$ candidate decays; events with $m_{\mu \mu}^{\mathrm{ID}}$ between 76 and $96 \mathrm{GeV}$ and the leading (sub-leading) muons with $26<p_{\mathrm{T}}^{\mathrm{ID}}<300 \mathrm{GeV}\left(15<p_{\mathrm{T}}^{\mathrm{ID}}<300 \mathrm{GeV}\right)$ are selected as $Z \rightarrow \mu \mu$ candidate decays. To enhance the sensitivity to the $p_{\mathrm{T}}$ dependent correction effects, the $m_{\mu \mu}^{\mathrm{ID}}$ is classified according to the $p_{\mathrm{T}}$ of the muons: for $J / \psi \rightarrow \mu \mu$ candidates the $p_{\mathrm{T}}^{\mathrm{ID}}$ of the sub-leading muon defines three bins with lower thresholds at $p_{\mathrm{T}}^{\mathrm{ID}}=8,9,11 \mathrm{GeV}$, for $Z \rightarrow \mu \mu$ candidates the $p_{\mathrm{T}}^{\mathrm{ID}}$ of the leading muon defines three bins with lower thresholds at $p_{\mathrm{T}}^{\mathrm{ID}}=26,47,70 \mathrm{GeV}$.

Similarly, the MS corrections are extracted using the distribution of the MS reconstructed dimuon invariant mass, $m_{\mu \mu}^{\mathrm{MS}}$, in the same way as for the ID. However, as in the MS part of Eq. (9) more correction parameters and more ROFs are present, an additional variable sensitive to the momentum scale and resolution is added to the MS fit. The variable, used only in $Z \rightarrow \mu \mu$ candidate events, is defined by the following equation:

$\rho=\frac{p_{\mathrm{T}}^{\mathrm{MS}}-p_{\mathrm{T}}^{\mathrm{ID}}}{p_{\mathrm{T}}^{\mathrm{ID}}}$, 
representing a measurement of the $p_{\mathrm{T}}$ imbalance between the measurement in the ID and in the MS. The $\rho$ variable is binned according to $p_{\mathrm{T}}^{\mathrm{MS}}$ of the muon in the ROF: the lower thresholds are $p_{\mathrm{T}}^{\mathrm{MS}}=20,30,35,40,45,55,70 \mathrm{GeV}$.

In order to compare the simulation to the data distributions, the corresponding templates of $m_{\mu \mu}^{\mathrm{ID}}, m_{\mu \mu}^{\mathrm{MS}}$, and $\rho$ are built using the MC samples of the $J / \psi \rightarrow \mu \mu$ and $Z \rightarrow \mu \mu$ signals. The background in the $Z \rightarrow \mu \mu$ mass region is added to the templates using the simulation and corresponds to approximately $0.1 \%$ of the $Z \rightarrow \mu \mu$ candidates. The nonresonant background to $J / \psi \rightarrow \mu \mu$, coming from decays of light and heavy hadrons and from Drell-Yan production, accounts for about $15 \%$ of the selected $J / \psi \rightarrow \mu \mu$ candidates. As it is not possible to accurately simulate it, a data driven approach is used to evaluate it: an analytic model of the background plus the $J / \psi$ signal is fitted to the dimuon mass spectrum of the $J / \psi \rightarrow \mu \mu$ candidates in a mass range 2.7$4.0 \mathrm{GeV}$, then the background model and its normalization are used in the template fit from which the momentum correction are extracted. The analytic fit is performed independently on the ID and MS event candidates. The non-resonant dimuon background is parametrized with an exponential function, while the $J / \psi$ and $\psi^{2 S}$ resonances are parametrized by a Crystal-Ball function [26] in the ID fits, or by a Gaussian distribution convoluted with a Landau in the MS fits, where energy loss effects due to the calorimeter material are larger.

The template fit machinery involves several steps: first a binned likelihood function $\mathcal{L}$ is built to compare the data to the MC templates of signal plus background. Then modified templates are generated by varying the correction parameters in Eq. (9) and applying them to the muon momentum of the simulated signal events. The $-2 \ln \mathcal{L}$ between data and the modified template is then minimized using MINUIT [27]. The procedure is iterated across all the ROFs: the first fit is performed using only events with both muons in the ROF, the following fits allow also one of the muons in a previously analysed ROF and one in the ROF under investigation. After all the detector ROFs have been analysed, the fit procedure is iterated twice in order to improve the stability of the results. The correction extraction is performed first for the ID and then for the MS, such that the ID transverse momentum present in Eq. (13) can be kept constant during the MS correction extraction.

Although the use of $p_{\mathrm{T}}$ bins for the construction of the templates gives a good sensitivity to the $p_{\mathrm{T}}$ dependence of the scale corrections, the fit is not very sensitive to the resolution correction terms $\Delta r_{0}^{\mathrm{MS}}(\eta, \phi)$ and $\Delta r_{2}^{\mathrm{MS}}(\eta, \phi)$ of Eq. (9). The reasons for this are, at low $p_{\mathrm{T}}$, the $p_{\mathrm{T}}>8 \mathrm{GeV}$ selection cut applied to the $J / \psi$ data sample, which limits the sensitivity to $\Delta r_{0}^{\mathrm{MS}}(\eta, \phi)$, and, at high $p_{\mathrm{T}}$, the limited statistics of the $Z \rightarrow \mu \mu$ data sample with $p_{\mathrm{T}}^{\mathrm{MS}}>100 \mathrm{GeV}$, which limits the sensitivity to $\Delta r_{2}^{\mathrm{MS}}(\eta, \phi)$. As the energy loss fluctuations do not show significant disagreement between data and MC for $|\eta|>0.8$, the parameter $\Delta r_{0}^{\mathrm{MS}}(\eta, \phi)$ has been fixed to zero in this region. The effect of the misalignment of MS chambers in real data, which is expected to be the largest contribution to $\Delta r_{2}^{\mathrm{MS}}(\eta, \phi)$, is already taken into account in the simulation as described in Sect. 3.2. Therefore the $\Delta r_{2}^{\mathrm{MS}}(\eta, \phi)$ term is also fixed to zero in the MS correction extraction. Two of the systematic uncertainties described in Sect. 5.1.2 are used to cover possible deviations from zero of these two terms.

\subsubsection{Systematic uncertainties}

Systematic uncertainties cover imperfections in the model used for the muon momentum correction and in the fit procedure used for the extraction of the correction terms. In particular the correction extraction procedure has been repeated using the following different configurations:

- variation of $\pm 5 \mathrm{GeV}$ in the dimuon mass window used for the $Z \rightarrow \mu \mu$ event selection. This is intended to cover resolution differences between data and MC that are beyond a simple Gaussian smearing. This results in one of the largest systematic uncertainties on the resolution corrections, with an average effect of $\approx 10 \%$ on the $\Delta r_{1}^{\mathrm{ID}}, \Delta r_{2}^{\mathrm{ID}}$, and $\Delta r_{1}^{\mathrm{MS}}$ parameters.

- Two variations of the $J / \psi$ templates used in the fit. The first concerns the $J / \psi$ background parametrization: new $m_{\mu \mu}^{\mathrm{MS}}$ and $m_{\mu \mu}^{\mathrm{ID}}$ background templates are generated using a linear model, for the MS fits, and a linear-timesexponential model, for the ID fits. The second variation concerns the $J / \psi$ event selection: the minimum muon $p_{\mathrm{T}}^{M S, I D}$ cut is raised from 8 to $10 \mathrm{GeV}$, thus reducing the weight of low- $p_{\mathrm{T}}$ muons on the corrections. The resulting variations on the resolution correction parameters are $\approx 10 \%$ of $\Delta r_{1}^{\mathrm{ID}}$ and $\Delta r_{1}^{\mathrm{MS}}$. The effect is also relevant for the MS scale corrections with a variation of $\approx 0.01 \mathrm{GeV}$ on $s_{0}^{\mathrm{MS}}$ and of $\approx 4 \times 10^{-4}$ on $s_{1}^{\mathrm{MS}}$.

- The ID correction extraction is repeated using $J / \psi \rightarrow \mu \mu$ events only or $Z \rightarrow \mu \mu$ events only. Since such configurations have a reduced statistical power, only the $s_{1}^{\mathrm{ID}}$ correction parameter is left free in the fit, while the resolution correction terms are fixed to nominal values. The resulting uncertainty on $s_{1}^{\mathrm{ID}}$, ranging from $0.01 \%$ to $0.05 \%$ from the central to the forward region of the ID, accounts for non-linear effects on the ID scale.

- The parameter $\Delta r_{0}^{\mathrm{MS}}$ of Eq. (9) is left free in all the regions, instead of fixing it to zero for $|\eta|>0.8$. The largest variation of $0.08 \mathrm{GeV}$ is applied as an additional systematic uncertainty on the parameter.

- The MS correction is extracted using a special $Z \rightarrow \mu \mu$ MC sample with ideal geometry, i.e. where no simulation of the misalignment of the MS chambers is applied. This is needed because the standard simulation has a too pessimistic resolution in the $|\eta|<1.25$ region, forcing the 
$\Delta r_{1}^{\mathrm{MS}}$ parameter to values compatible with zero. The template fit performed with the ideal-geometry $Z \rightarrow \mu \mu \mathrm{MC}$ sample gives $\Delta r_{1}^{\mathrm{MS}}>0$ in the region $0.4<|\eta|<1.25$. The largest variation of $\Delta r_{1}^{\mathrm{MS}}$, corresponding to 0.012 , is applied as an additional systematic uncertainty for this region.

- Variation of the normalization of the MC samples used in $Z \rightarrow \mu \mu$ background estimate by factors of two and one half. The resulting systematic uncertainty is small except for the detector regions with $|\eta|>2.0$, where the effect is comparable to the other uncertainties.

Independently from the fit procedure, the following studies are used to derive additional systematic uncertainties:

- The simulation of the ID includes an excess of material for $|\eta|>2.3$ resulting in a muon momentum resolution with is too pessimistic. Such imperfection is covered by adding a systematic uncertainties of $2 \times 10^{-3}$ on the $s_{1}^{\mathrm{ID}}$ parameter, and of 0.01 on the $\Delta r_{1}^{\mathrm{ID}}$ parameter, both for $|\eta|>2.3$. These are the largest systematic uncertainties on the ID correction parameters.

- The position of the mass peak in the $Z \rightarrow \mu \mu$ sample is studied in finer $\eta$ bins than those used to extract the corrections, using the fit that will be discussed in Sect. 5.2 as an alternative to the template fitting method. An additional uncertainty of $2 \times 10^{-4}$ on the $s_{1}^{\mathrm{ID}}(\eta)$ parameter is found to cover all the observed deviations between data and corrected MC.

- The effect of the measurement of the angle of the muon tracks has been checked by using the $J / \psi \mathrm{MC}$ and conservatively increasing the track angular resolution by $\approx 40 \%$. The maximum effect is an increase of the resolution correction $\Delta r_{1}^{\mathrm{ID}}$ of 0.001 , which is added to the systematic uncertainties.

- Special runs with the toroidal magnetic field off have been used to evaluate the quality of the MS chamber alignment. These results are compared to the chamber misalignments in the simulation to define the systematic uncertainty on the $\Delta r_{2}^{\mathrm{MS}}(\eta, \phi)$ resolution correction parameter.

The final uncertainty on each of the eight muon momentum correction parameters is derived from the sum in quadrature of all the listed uncertainty sources. This is simplified for use in standard physics analyses, for which only four systematic variations are provided: global upper and lower scale variations and independent resolution variations for the ID and the MS. The upper and lower scale variations are obtained by a simultaneous variation of all the ID and MS scale correction parameters by $1 \sigma$. The resolution variation for ID (MS) is obtained by the simultaneous variation of all the ID (MS) correction parameters.
Table 1 Summary of ID muon momentum resolution and scale corrections used in Eq. (9), averaged over three main detector regions. The corrections are derived in $18 \eta$ detector regions, as described in Sect. 5.1.1, and averaged according to the $\eta$ width of each region. The uncertainties are the result of the sum in quadrature of the statistical and systematic uncertainties. Only upper uncertainties are reported for the $\Delta r$ parameters; lower uncertainties are evaluated by symmetrization, as described in Sect. 5.1.2

\begin{tabular}{llll}
\hline Region & $\Delta r_{1}^{\mathrm{ID}}$ & $\Delta r_{2}^{\mathrm{ID}}\left[\mathrm{TeV}^{-1}\right]$ & $s_{1}^{\mathrm{ID}}$ \\
\hline$|\eta|<1.05$ & $0.0068^{+0.0010}$ & $0.146^{+0.039}$ & $-0.92_{-0.22}^{+0.26} \times 10^{-3}$ \\
$1.05 \leq|\eta|<2.0$ & $0.0105^{+0.0018}$ & $0.302^{+0.046}$ & $-0.86_{-0.35}^{+0.30} \times 10^{-3}$ \\
$|\eta| \geq 2.0$ & $0.0069^{+0.0121}$ & $0.088^{+0.084}$ & $-0.49_{-1.63}^{+1.17} \times 10^{-3}$ \\
\hline
\end{tabular}

The MC-smearing approach of Eq. (9) cannot be used to correct the $\mathrm{MC}$ when the resolution in real data is better than in the simulation. To deal with these cases, the amount of resolution that should be subtracted in quadrature from the simulation to reproduce the data is included in the positive ID and MS resolution variations. Then the prescription for physics analysis is to symmetrize the effect of the positive variation of resolution parameters around the nominal value of the physical observables under study.

\subsubsection{Result of the muon momentum scale and resolution corrections}

The ID and MS correction parameters used in Eq. (9) are shown in Tables 1 and 2, averaged over three $\eta$ regions. The scale correction to the simulated ID track reconstruction is always below $0.1 \%$ with an uncertainty ranging from $\approx 0.02 \%$, for $|\eta|<1.0$, to $0.2 \%$, for $|\eta|>2.3$. The correction to the MS scale is $\lesssim 0.1 \%$ except for the large MS sectors in the barrel region of the detector, where a correction of $\approx 0.3 \%$ is needed, and for specific MS regions with $1.25<|\eta|<1.5$ where a correction of about $-0.4 \%$ is needed. An energy loss correction of approximately $30 \mathrm{MeV}$ is visible for low values of $p_{\mathrm{T}}$ in the MS reconstruction. This correction corresponds to about $1 \%$ of the total energy loss in the calorimeter and in the dead material in front of the spectrometer and is compatible with the accuracy of the material budget used in the simulation. Depending on the considered $p_{\mathrm{T}}$ range, total resolution smearing corrections below $10 \%$ and below $15 \%$ are needed for the simulated ID and MS track reconstructions.

\subsection{Measurement of the dimuon mass scale and resolution}

The collected samples of $J / \psi \rightarrow \mu \mu, \Upsilon \rightarrow \mu \mu$ and $Z \rightarrow$ $\mu \mu$ decays have been used to study the muon momentum resolution and to validate the momentum corrections obtained with the template fit method described in the previous section with a different methodology. In addition the $\Upsilon$ sample, not 
Table 2 Summary of MS momentum resolution and scale corrections for small and large MS sectors, averaged over three main detector regions. The corrections for large and small MS sectors are derived in 18 $\eta$ detector regions, as described in Sect. 5.1.1, and averaged according to the $\eta$ width of each region. The parameters $\Delta r_{0}^{\mathrm{MS}}$, for $|\eta|>1.05$, and

\begin{tabular}{llllll}
\hline Region & $\Delta r_{0}^{\mathrm{MS}}[\mathrm{GeV}]$ & $\Delta r_{1}^{\mathrm{MS}}$ & $\Delta r_{2}^{\mathrm{MS}}\left[\mathrm{TeV}^{-1}\right]$ & $s_{0}^{\mathrm{MS}}[\mathrm{GeV}]$ & $s_{1}^{\mathrm{MS}}$ \\
\hline$|\eta|<1.05$ (small) & $0.115^{+0.083}$ & $0.0030^{+0.0079}$ & $0^{+0.21}$ & $-0.035_{-0.011}^{+0.017}$ & $+3.57_{-0.60}^{+0.38} \times 10^{-3}$ \\
$|\eta|<1.05$ (large) & $0.101^{+0.090}$ & $0.0034^{+0.0081}$ & $0^{+0.11}$ & $-0.022_{-0.014}^{+0.007}$ & $-0.22_{-0.24}^{+0.37} \times 10^{-3}$ \\
$1.05 \leq|\eta|<2.0$ (small) & $0^{+0.080}$ & $0.0171^{+0.0059}$ & $0^{+0.22}$ & $-0.032_{-0.016}^{+0.017}$ & $-1.07_{-0.93}^{+0.77} \times 10^{-3}$ \\
$1.05 \leq|\eta|<2.0$ (large) & $0^{+0.080}$ & $0.0190^{+0.0047}$ & $0^{+0.17}$ & $-0.026_{-0.017}^{+0.009}$ & $-1.46_{-0.57}^{+0.45} \times 10^{-3}$ \\
$|\eta| \geq 2.0$ (small) & $0^{+0.080}$ & $0.0022^{+0.0075}$ & $0^{+0.06}$ & $-0.031_{-0.031}^{+0.029}$ & $-0.91_{-0.91}^{+1.63} \times 10^{-3}$ \\
$|\eta| \geq 2.0$ (large) & $0^{+0.080}$ & $0.0171^{+0.0052}$ & $0^{+0.29}$ & $-0.057_{-0.021}^{+0.019}$ & $+0.40_{-0.50}^{+1.22} \times 10^{-3}$ \\
\hline
\end{tabular}

$\Delta r_{2}^{\mathrm{MS}}$, for the full $\eta$ range, are fixed to zero. The uncertainties are the result of the sum in quadrature of the statistical and systematic uncertainties. Only upper uncertainties are reported for the $\Delta r$ parameters; lower uncertainties are evaluated by symmetrization, as described in Sect. 5.1.2 used in the extraction of the corrections, provides an independent validation.

Neglecting angular effects, the invariant mass resolution $\sigma\left(m_{\mu \mu}\right)$ is related to the momentum resolution by

$$
\frac{\sigma\left(m_{\mu \mu}\right)}{m_{\mu \mu}}=\frac{1}{2} \frac{\sigma\left(p_{1}\right)}{p_{1}} \oplus \frac{1}{2} \frac{\sigma\left(p_{2}\right)}{p_{2}},
$$

where $p_{1}$ and $p_{2}$ are the momenta of the two muons. If the momentum resolution is similar for the two muons then the relative mass resolution is proportional to the relative momentum resolution:

$\frac{\sigma\left(m_{\mu \mu}\right)}{m_{\mu \mu}}=\frac{1}{\sqrt{2}} \frac{\sigma(p)}{p}$.

The mass resolution has been obtained by fitting the width of the invariant mass peaks. In the $J / \psi \rightarrow \mu \mu$ and $\Upsilon \rightarrow \mu \mu$ decays, the intrinsic width of the resonance is negligible with respect to the experimental resolution. In the $Z \rightarrow \mu \mu$ case the fits have been performed using a convolution of the true line-shape obtained from the MC simulation with an experimental resolution function. The momentum scale was obtained by comparing the mass peak position in data and in MC. Details of the event selection and of the invariant mass fits are given below.

\subsubsection{Event selection and mass fitting}

The $J / \psi$ and $\Upsilon$ events are selected online by the dedicated dimuon triggers described in Sect. 3.1. The offline event selection requires in addition that both muons are reconstructed as $\mathrm{CB}$ muons and have $p_{\mathrm{T}}>7 \mathrm{GeV}$. The trigger acceptance limits the muons to the region $|\eta|<2.4$. The resulting data samples consist of $17 \mathrm{M}$ and $4.7 \mathrm{M}$ candidates for $J / \psi$ and $\Upsilon$, respectively. The $Z \rightarrow \mu \mu$ sample was selected online with the single-muon trigger described in Sect. 4.1. One of the two muons can be outside the trigger acceptance, allowing coverage of the full range $|\eta|<2.7$. The offline selection requires two opposite-charge muons, one with $p_{\mathrm{T}}>25 \mathrm{GeV}$ and one with $p_{\mathrm{T}}>20 \mathrm{GeV}$. The two muons are required to be isolated, to have opposite charges and to be compatible with the primary interaction vertex.

The invariant mass distribution of the $J / \psi \rightarrow \mu \mu$, $\Upsilon \rightarrow \mu \mu$ and $Z \rightarrow \mu \mu$ samples are shown in Fig. 10 and compared with uncorrected and corrected MC. With the uncorrected MC the signal peaks have smaller width and are slightly shifted with respect to data. After correction, the lineshapes of the three resonances agree very well with the data. For a detailed study, the position $\left\langle m_{\mu \mu}\right\rangle$ and the width $\sigma\left(m_{\mu \mu}\right)$ of the mass peaks are extracted in bins of $\eta$ and $p_{\mathrm{T}}$ from fits of the invariant mass distributions of the three resonances.

In the $J / \psi$ case, for each bin, the background is obtained from a fit of two sideband regions outside the $J / \psi$ mass peak $\left(2.55<m_{\mu \mu}<2.9\right.$ and $\left.3.3<m_{\mu \mu}<4.0 \mathrm{GeV}\right)$ using a second order polynomial. The background is then subtracted from the signal mass window. The parameters $\left\langle m_{\mu \mu}\right\rangle$ and $\sigma\left(m_{\mu \mu}\right)$ of the background subtracted signal distribution are obtained with a Gaussian fit in the range $\left\langle m_{\mu \mu}\right\rangle \pm 1.5 \sigma\left(m_{\mu \mu}\right)$, obtained using an iterative procedure. Systematic uncertainties associated to the fit are evaluated by repeating the fit using a third order polynomial as the background model and by varying the fit range to $\pm 1 \times$ and $\pm 2 \times \sigma\left(m_{\mu \mu}\right)$.

As shown in Fig. 10, the three $\Upsilon$ resonances $(1 \mathrm{~S}, 2 \mathrm{~S}$, $3 \mathrm{~S})$ partially overlap. Moreover in the $\Upsilon$ case the mass window imposed by the trigger limits considerably the size of the sidebands available for fixing the background level. Therefore a different fit strategy is adopted in this case. For each bin, the whole invariant mass distribution in the range $8.5<m_{\mu \mu}<11.5 \mathrm{GeV}$ is fitted with a linear background plus three Crystal-Ball functions representing the three resonances. The $\alpha$ and $n$ parameters that fix the tail of the CrystalBall function are fixed to the values obtained from a fit of the signal MC mass distribution. The relative mass shifts of 

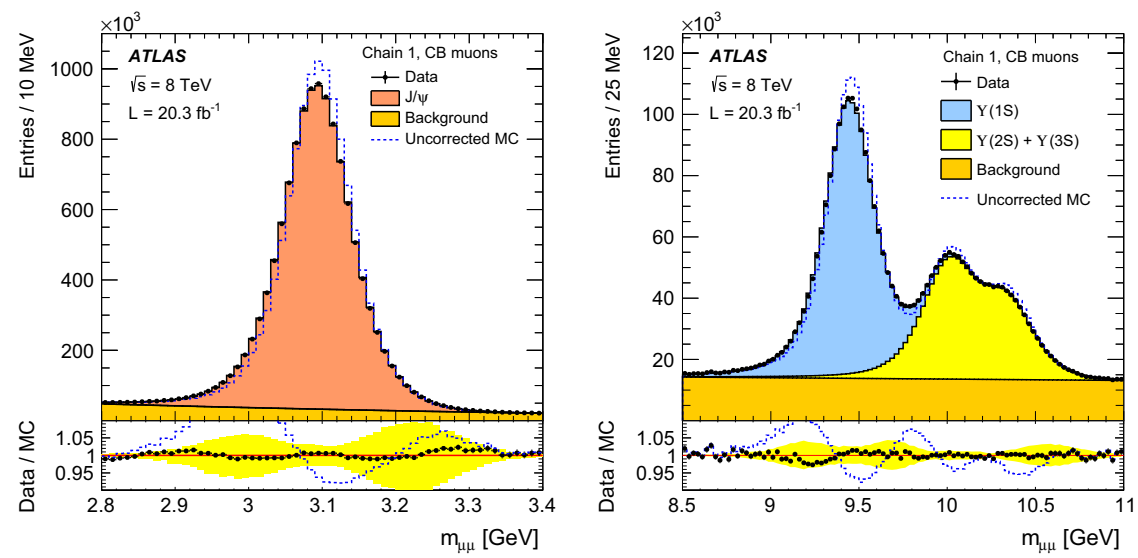

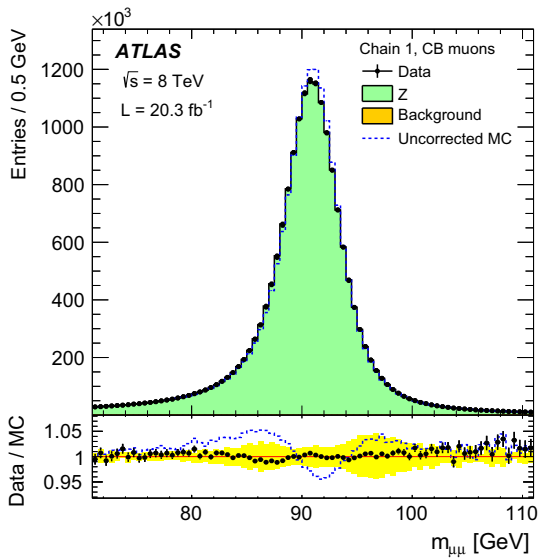

Fig. 10 Dimuon invariant mass distribution of $J / \psi \rightarrow \mu \mu$ (left), $\Upsilon \rightarrow \mu \mu$ (center) and $Z \rightarrow \mu \mu$ (right) candidate events reconstructed with $\mathrm{CB}$ muons. The upper panels show the invariant mass distribution for data and for the signal MC simulation plus the background estimate. The points show the data, the filled histograms show the simulation with the MC momentum corrections applied and the dashed histogram shows the simulation when no correction is applied. Background estimates are added to the signal simulation. The lower panels show the Data/MC ratios. The band represents the effect of the systematic uncertainties on the MC momentum corrections. In the $J / \psi$ case the background was fitted in a sideband region as described in the text. In the $\Upsilon$ case a simultaneous fit of the normalization of the three simulated $\Upsilon \rightarrow \mu \mu$ distributions and of a linear background was performed. In the $Z$ case, the $\mathrm{MC}$ background samples are added to the signal sample according to their expected cross sections. The sum of background and signal MC is normalized to the data the three signal peaks are fixed using the PDG masses of the three resonances, while the widths of the three peaks, divided by the corresponding PDG masses, are constrained to be equal. The remaining free parameters in the fit are the mass scale, the width $\sigma\left(m_{\mu \mu}\right)$ of the $\Upsilon(1 S)$, the relative normalizations of the $\Upsilon(2 S)$ and $\Upsilon(3 S)$ distributions with respect to $\Upsilon(1 S)$ and two parameters for the linear background. A similar fit is performed on the MC simulation of the invariant mass distribution obtained by adding the three signal peaks and a flat background distribution. The fit systematic uncertainties have been evaluated by chaining the fit range to $8.25<m_{\mu \mu}<11.75$ and $8.75<m_{\mu \mu}<11.0 \mathrm{GeV}$ and by varying the $\alpha$ and $n$ parameters in the range allowed by fits to the simulation.

In the $Z \rightarrow \mu \mu$ case, for each bin, the true lineshape predicted by the MC simulation is parametrized with a BreitWigner function. The measured dimuon mass spectrum is fitted with a Crystal-Ball function, representing the experimental resolution effects, convoluted with the Breit-Wigner parametrization of the true lineshape. The fit is repeated in different ranges around the mass peak (corresponding approximately to one to two standard deviations) and the spread of the results is used to evaluate the systematic uncertainty of the fit.

\subsubsection{Mass scale results}

Figure 11 shows the Data/MC ratio of the mean mass $\left\langle m_{\mu \mu}\right\rangle$ obtained from the fits to the $Z, J / \psi, \Upsilon$ samples described above, as a function of the pseudorapidity of the highest- $p_{\mathrm{T}}$

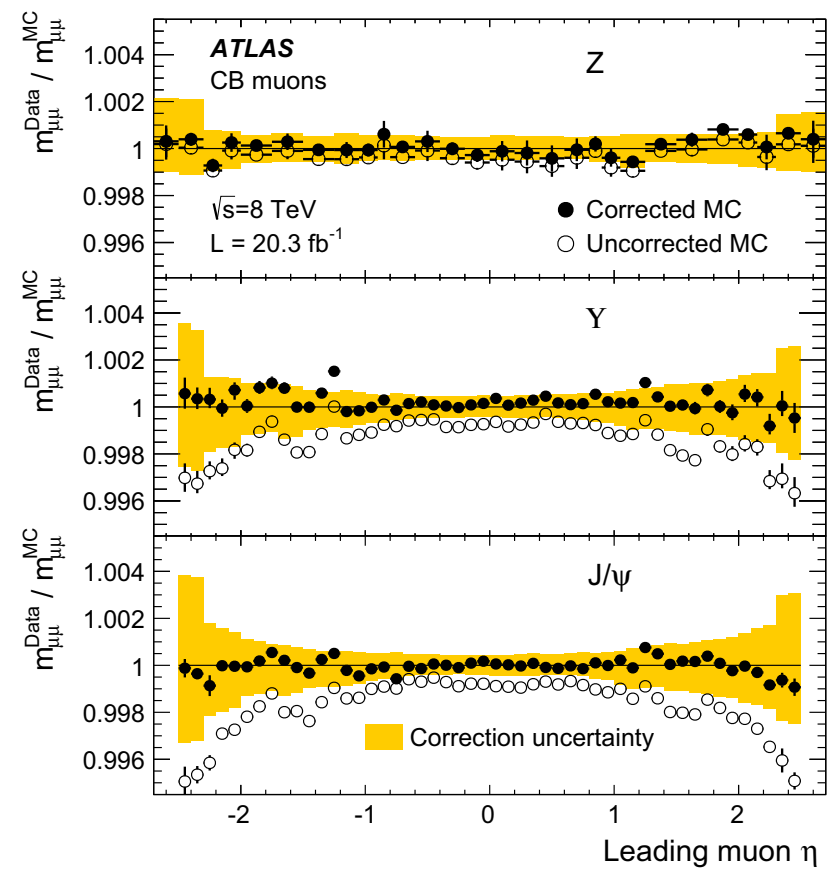

Fig. 11 Ratio of the fitted mean mass, $\left\langle m_{\mu \mu}\right\rangle$, for data and corrected MC from $Z$ (top), $\Upsilon$ (middle), and $J / \psi$ (bottom) events as a function of the pseudorapidity of the highest- $p_{\mathrm{T}}$ muon. The ratio is shown for corrected MC (filled symbols) and uncorrected MC (empty symbols). The error bars represent the statistical and the systematic uncertainty on the mass fits added in quadrature. The bands show the uncertainty on the MC corrections calculated separately for the three samples

muon for pairs of $\mathrm{CB}$ muons. For the uncorrected $\mathrm{MC}$, the ratio deviates from unity in the large $|\eta|$ region of the $J / \psi$ and $\Upsilon$ cases by up to $5 \%$. This is mainly due to imperfections 

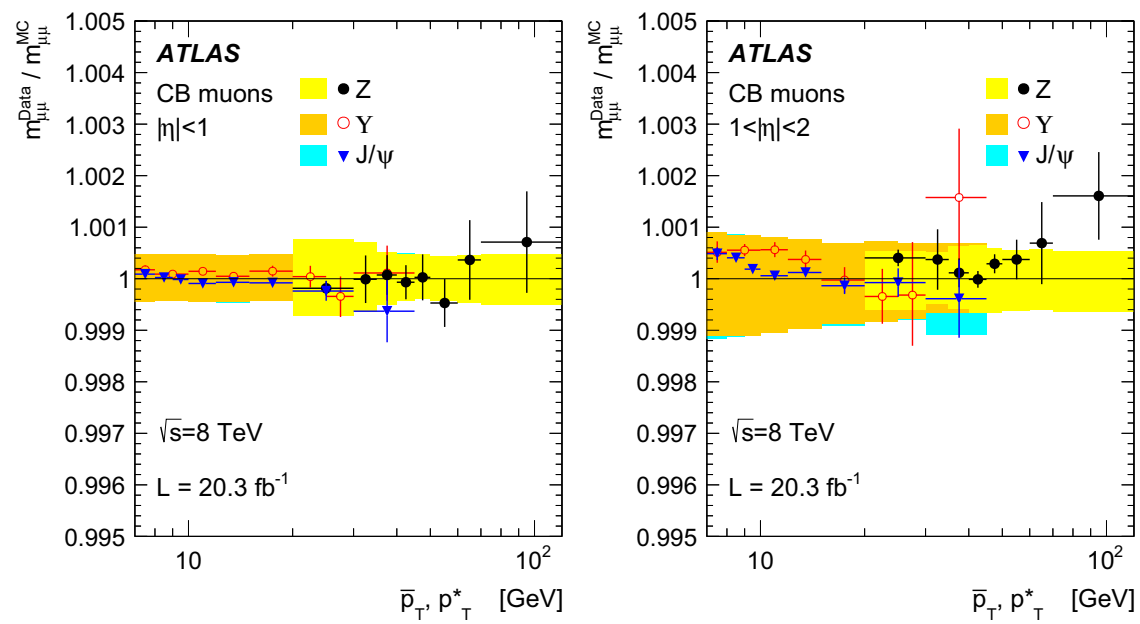

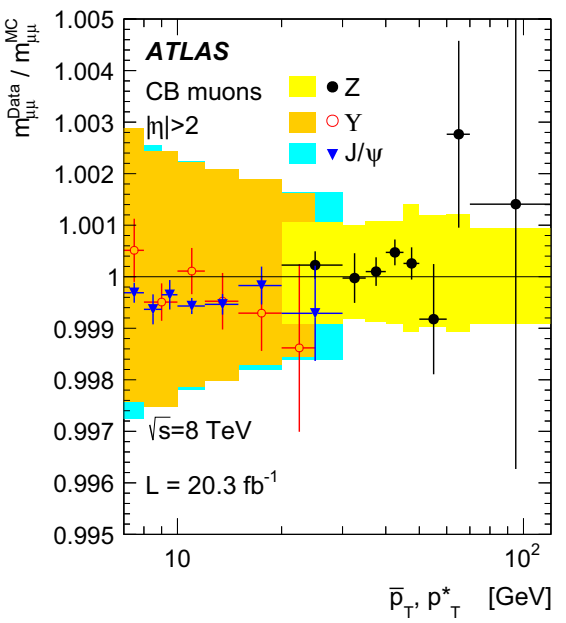

Fig. 12 Ratio of the fitted mean mass, $\left\langle m_{\mu \mu}\right\rangle$, for data and corrected MC from $J / \psi, \Upsilon$ and $Z$ events as a function of the average transverse momentum in three $|\eta|$ ranges. Both muons are required to be in the same $|\eta|$ range. The $J / \psi$ and $\Upsilon$ data are shown as a function of the $\bar{p}_{\mathrm{T}}=\frac{1}{2}\left(p_{\mathrm{T}, 1}+p_{\mathrm{T}, 2}\right)$ while for $Z$ data are plotted as a function of $p_{\mathrm{T}}^{*}$ as

in the simulation of the muon energy loss that have a larger effect at low $p_{\mathrm{T}}$ and in the forward $\eta$ region where the MS measurement has a larger weight in the MS-ID combination. The corrected MC is in very good agreement with the data, well within the scale systematics that are $\approx 0.035 \%$ in the barrel region and increase with $|\eta|$ to reach $\sim 0.2 \%$ in the region $|\eta|>2$ for the $Z \rightarrow \mu \mu$ case.

Figure 12 shows the data/MC ratio for $\left\langle m_{\mu \mu}\right\rangle$ as a function of the transverse momentum $\left\langle p_{\mathrm{T}}\right\rangle$ for muons in three different pseudorapidity regions.

For the $J / \psi$ and $\Upsilon$ cases, $\left\langle p_{\mathrm{T}}\right\rangle$ is defined as the average momentum $\bar{p}_{T}=\frac{1}{2}\left(p_{\mathrm{T}, 1}+p_{\mathrm{T}, 2}\right)$ while in the $Z$ case it is defined as

$p_{\mathrm{T}}^{*}=m_{Z} \sqrt{\frac{\sin \theta_{1} \sin \theta_{2}}{2\left(1-\cos \alpha_{12}\right)}}$,

where $m_{Z}$ is the $Z$ pole mass [28], $\theta_{1}, \theta_{2}$ are the polar angles of the two muons and $\alpha_{12}$ is the opening angle of the muon pair. This definition, based on angular variables only, removes the correlation between the measurement of the dimuon mass and of the average $p_{\mathrm{T}}$ that is particularly relevant around the Jacobian peak at $p_{\mathrm{T}}=m_{Z} / 2$ in the distribution of muons from $Z$ decays.

The data from the three resonances span from $\left\langle p_{\mathrm{T}}\right\rangle=$ $7 \mathrm{GeV}$ to $\left\langle p_{\mathrm{T}}\right\rangle=120 \mathrm{GeV}$ and show that the momentum scale is well known and within the assigned systematic uncertainties in the whole $p_{\mathrm{T}}$ range.

\subsubsection{Resolution results}

The dimuon mass width $\sigma\left(m_{\mu \mu}\right)$ for CB muons is shown as a function of the leading-muon $\eta$ in Fig. 13 for the three reso- defined in Eq. (16). The error bars represent the statistical uncertainty and the systematic uncertainty on the fit added in quadrature. The bands show the uncertainty on the MC corrections calculated separately for the three samples

nances. The width of the uncorrected MC is $5-10 \%$ smaller than that of the data. After correction the MC reproduces the width of the data well within the correction uncertainties.

At a given $\eta$, the relative dimuon mass resolution $\sigma\left(m_{\mu \mu}\right) / m_{\mu \mu}$ depends approximately on $\left\langle p_{\mathrm{T}}\right\rangle$ (Eq. 15). This allows a direct comparison of the momentum resolution using different resonances. This is shown in Fig. 14, where the relative mass resolution from $J / \psi \rightarrow \mu \mu, \Upsilon \rightarrow \mu \mu$ and $Z \rightarrow \mu \mu$ events is compared in three regions of $|\eta|$. The $J / \psi \rightarrow \mu \mu$ and $\Upsilon \rightarrow \mu \mu$ resolutions are in good agreement.

In the $Z \rightarrow \mu \mu$ sample, due to the decay kinematics, below $\left\langle p_{\mathrm{T}}\right\rangle=m_{Z} / 2$ there is a strong correlation between $\left\langle p_{\mathrm{T}}\right\rangle$ and the pseudorapidity of the muons, in such a way that the lower is the $\left\langle p_{\mathrm{T}}\right\rangle$, the larger is the $|\eta|$ of the muons. Above $\left\langle p_{\mathrm{T}}\right\rangle=m_{Z} / 2$, the correlation effect is strongly reduced and the $Z$ measurements are well aligned with those from the lighter resonances. In the barrel region, $|\eta|<1$, the mass resolution increases from $\sigma\left(m_{\mu \mu}\right) / m_{\mu \mu} \approx 1.2 \%$ at $p_{\mathrm{T}}<$ $10 \mathrm{GeV}$ to $\sigma\left(m_{\mu \mu}\right) / m_{\mu \mu} \approx 2 \%$ at $p_{\mathrm{T}}=100 \mathrm{GeV}$. For $|\eta|>1$ it goes from $\sigma\left(m_{\mu \mu}\right) / m_{\mu \mu} \approx 2 \%$ to $\approx 3 \%$ in the same $p_{\mathrm{T}}$ range. This behavior is very well reproduced by the corrected MC. Following Eq. (15), it is possible to scale $\sigma\left(m_{\mu \mu}\right) / m_{\mu \mu}$ by $\sqrt{2}$ to extract a measurement of the relative momentum resolution $\sigma(p) / p$, which ranges from $\approx 1.7 \%$ in the central region and at low $p_{\mathrm{T}}$ to $\approx 4 \%$ at large $\eta$ and $p_{\mathrm{T}}=100 \mathrm{GeV}$.

To understand better the $p_{\mathrm{T}}$ dependence of the momentum resolution of CB muons, it is useful to study separately the resolution of the ID and of the MS measurements, as shown in Figs. 15 and 16. The ID measurement has a better resolution than the MS in the $p_{\mathrm{T}}$ range under study for $|\eta|<2$ while 


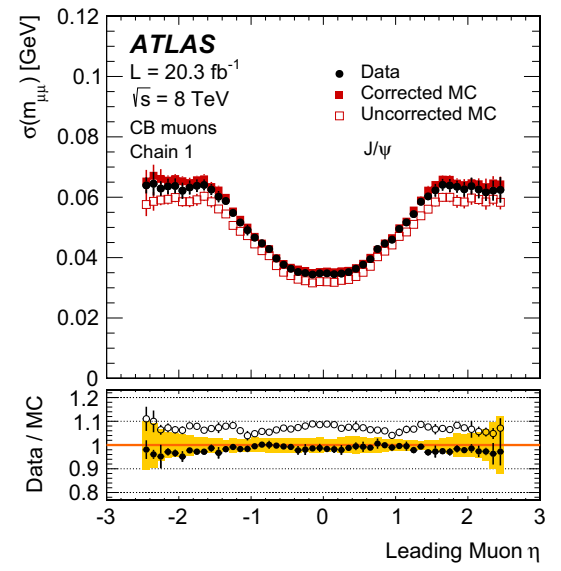

(a)

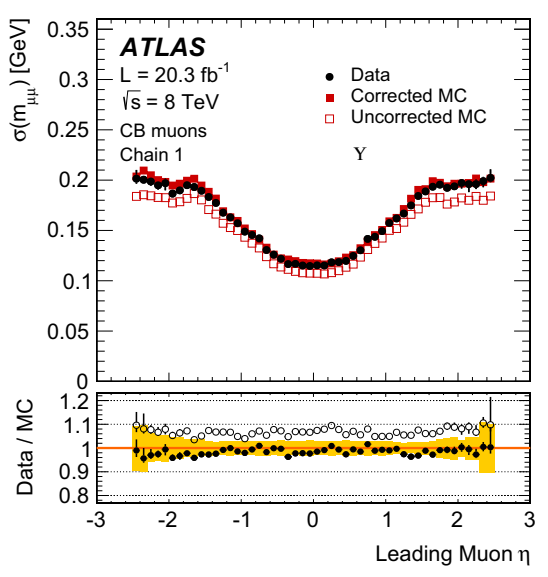

(b)

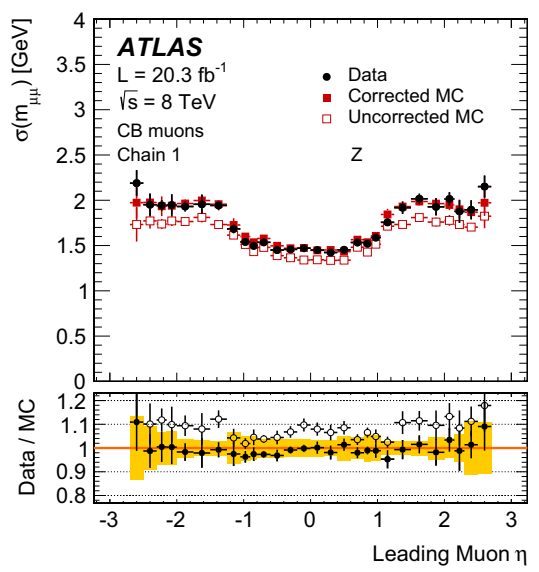

(c)
Fig. 13 Dimuon invariant mass resolution for $\mathrm{CB}$ muons for $J / \psi \rightarrow \mu \mu(\mathbf{a}), \Upsilon \rightarrow \mu \mu(\mathbf{b})$ and $Z \rightarrow \mu \mu(\mathbf{c})$ events for data and for uncorrected and corrected $\mathrm{MC}$ as a function of the pseudorapidity of the highest- $p_{\mathrm{T}}$ muon. The upper plots show the fitted resolution parameter for data, uncorrected MC and corrected MC. The lower panels show the data/MC ratio, using uncorrected and corrected $\mathrm{MC}$. The error bars represent the statistical uncertainty and the systematic uncertainty on the fit added in quadrature. The bands in the lower panels represent the systematic uncertainty on the correction
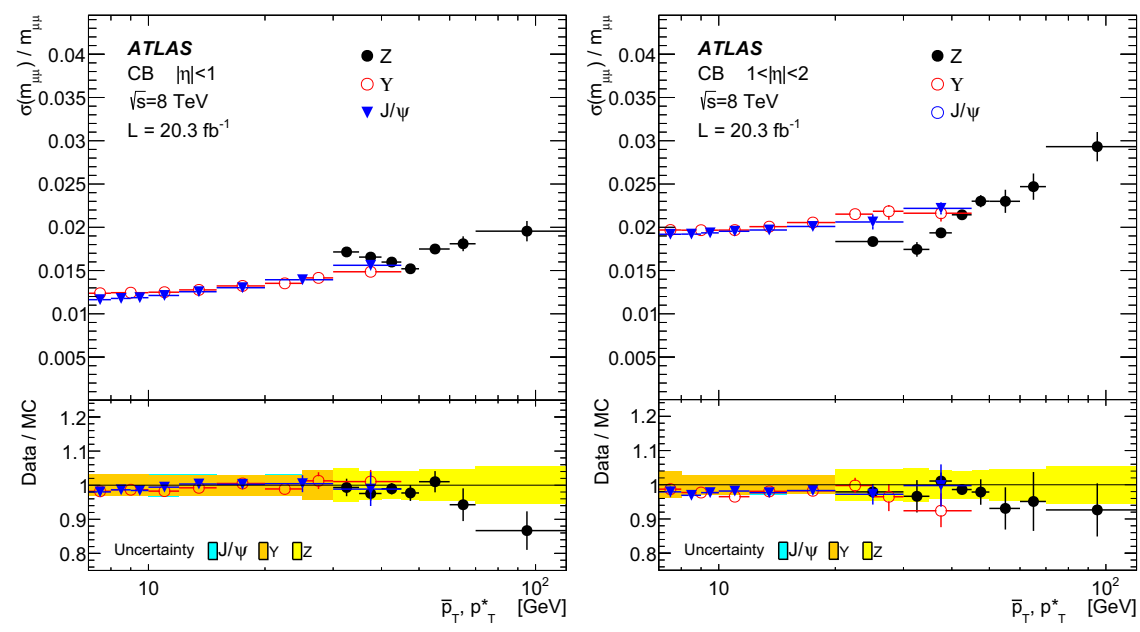

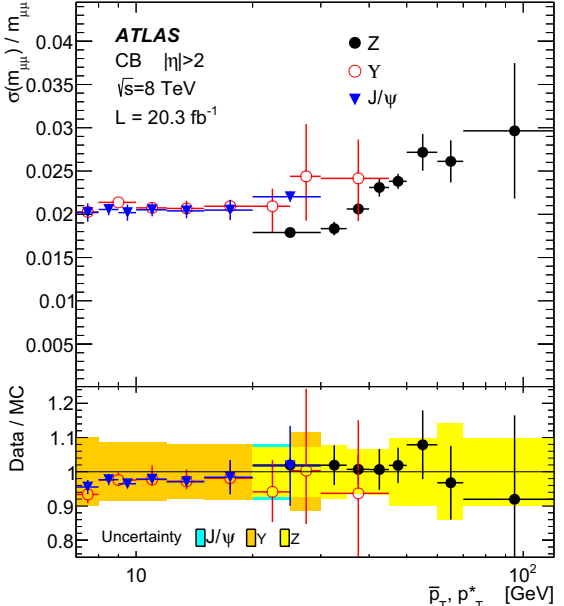

Fig. 14 Dimuon invariant mass resolution for CB muons measured from $J / \psi, \Upsilon$ and $Z$ events as a function of the average transverse momentum in three $|\eta|$ ranges. Both muons are required to be in the same $|\eta|$ range. The $J / \psi$ and $\Upsilon$ data are plotted as a function of $\bar{p}_{\mathrm{T}}=\frac{1}{2}\left(p_{\mathrm{T}, 1}+p_{\mathrm{T}, 2}\right)$ while for $Z$ data are plotted as a function of $p_{\mathrm{T}}^{*}$ as

the MS has a better resolution at larger $|\eta|$. The resolution of the CB muons is significantly better than the ID or the MS measurements taken separately in the whole $|\eta|$ range. The ID resolution has an approximately linear increases with $p_{\mathrm{T}}$, corresponding to a non-zero $r_{2}$ term in Eq. (10). The MS resolution is largest in the region $1<|\eta|<2$ which contains the areas with the lowest magnetic field integral. In the region $|\eta|<1$ there is a visible increase at low $p_{\mathrm{T}}$ that corresponds to the presence of a non-zero $r_{0}$ term in Eq. (10). The $p_{\mathrm{T}}$ dependence of the resolutions for both the ID and the MS measurements is well reproduced by the corrected MC. defined in Eq. (16). The error bars represent statistical and systematic errors added in quadrature. The lower panel shows the ratio between data and the corrected MC, with bands representing the uncertainty on the $\mathrm{MC}$ corrections for the three calibration samples

According to studies based on MC, the MS measurement is expected to dominate over the ID in the whole $|\eta|$ range for sufficiently large $p_{\mathrm{T}}$.

\section{Final state radiation recovery}

The invariant mass distributions of resonances that decay into muons, such as $Z \rightarrow \mu \mu$ and $H \rightarrow Z Z \rightarrow 4 \ell$, is affected by QED final state radiation of photons, causing the mass reconstructed using muons to be shifted to lower values. 

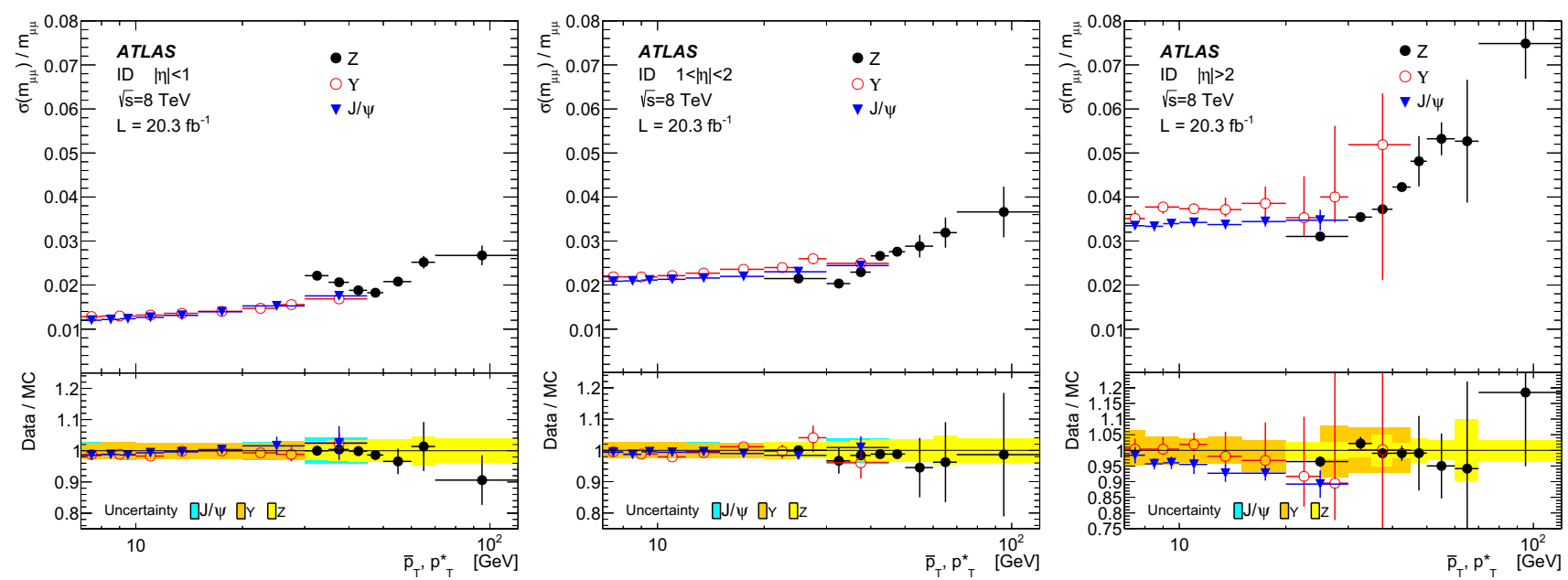

Fig. 15 Dimuon invariant mass resolution for muons reconstructed with the ID only, measured from $J / \psi, \Upsilon$ and $Z$ events as a function of the average transverse momentum in three $|\eta|$ ranges. Other details as in Fig. 14
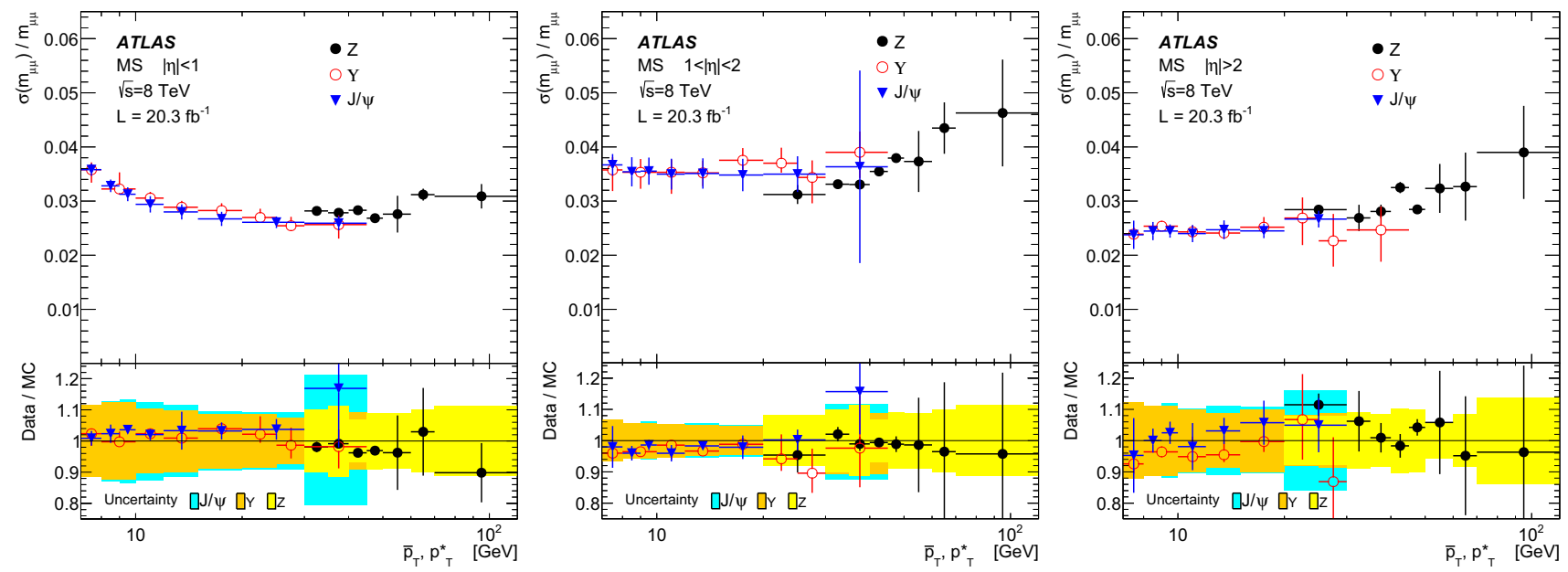

Fig. 16 Dimuon invariant mass resolution for muons reconstructed with the MS only, measured from $J / \psi, \Upsilon$ and $Z$ events as a function of the average transverse momentum in three $|\eta|$ ranges. Other details as in Fig. 14

In this section, a dedicated method to include FSR photons in the reconstruction of resonances decaying into muons is introduced and tested with $Z \rightarrow \mu \mu$ data. This method has been used in several ATLAS publications [6,29].

Final state radiation photons emitted collinearly to muons can be reconstructed with the LAr calorimeter: electromagnetic clusters are searched for within a narrow cone around the axis defined by the muon momentum direction at the interaction point (i.e. the direction which would be followed by an uncharged particle). The longitudinal segmentation of the LAr calorimeter is exploited to reduce fake photon clusters produced by muon energy losses in the calorimeter. This is achieved by using as a discriminant the fraction $\mathrm{f}_{1}$ of the cluster energy deposited in the first segment of the calorimeter divided by the total cluster energy. Collinear FSR photon candidates are required to have $E_{T}>1.5 \mathrm{GeV}$, $\Delta R_{\text {cluster, } \mu}<0.15$ and $\mathrm{f}_{1}>0.1$. In addition, non-collinear
FSR photons are recovered using the standard ATLAS photon reconstruction, selecting isolated photons emitted with $\Delta R_{\text {cluster, } \mu}>0.15$ and with $E_{T}>10 \mathrm{GeV}$ [30].

The effect of adding a collinear or non-collinear FSR photon to the $Z \rightarrow \mu \mu$ invariant mass in data is studied in a sample obtained with a dedicated selection of $Z \rightarrow \mu \mu$ candidates plus at least one radiated photon candidate.

The correction for collinear FSR is applied for events in the mass window $66 \mathrm{GeV}<m_{\mu \mu}<89 \mathrm{GeV}$ while the correction for non-collinear FSR photons is applied only if the collinear search has failed and the dimuon mass satisfies $m_{\mu \mu}<81 \mathrm{GeV}$.

In Fig. 17 the invariant mass distributions for the sample of $Z \rightarrow \mu \mu$ events with a FSR photon candidate are shown before and after the addition of collinear and non-collinear FSR photons. A good agreement between data and MC is observed for the corrected $Z \rightarrow \mu \mu$ events. According to 

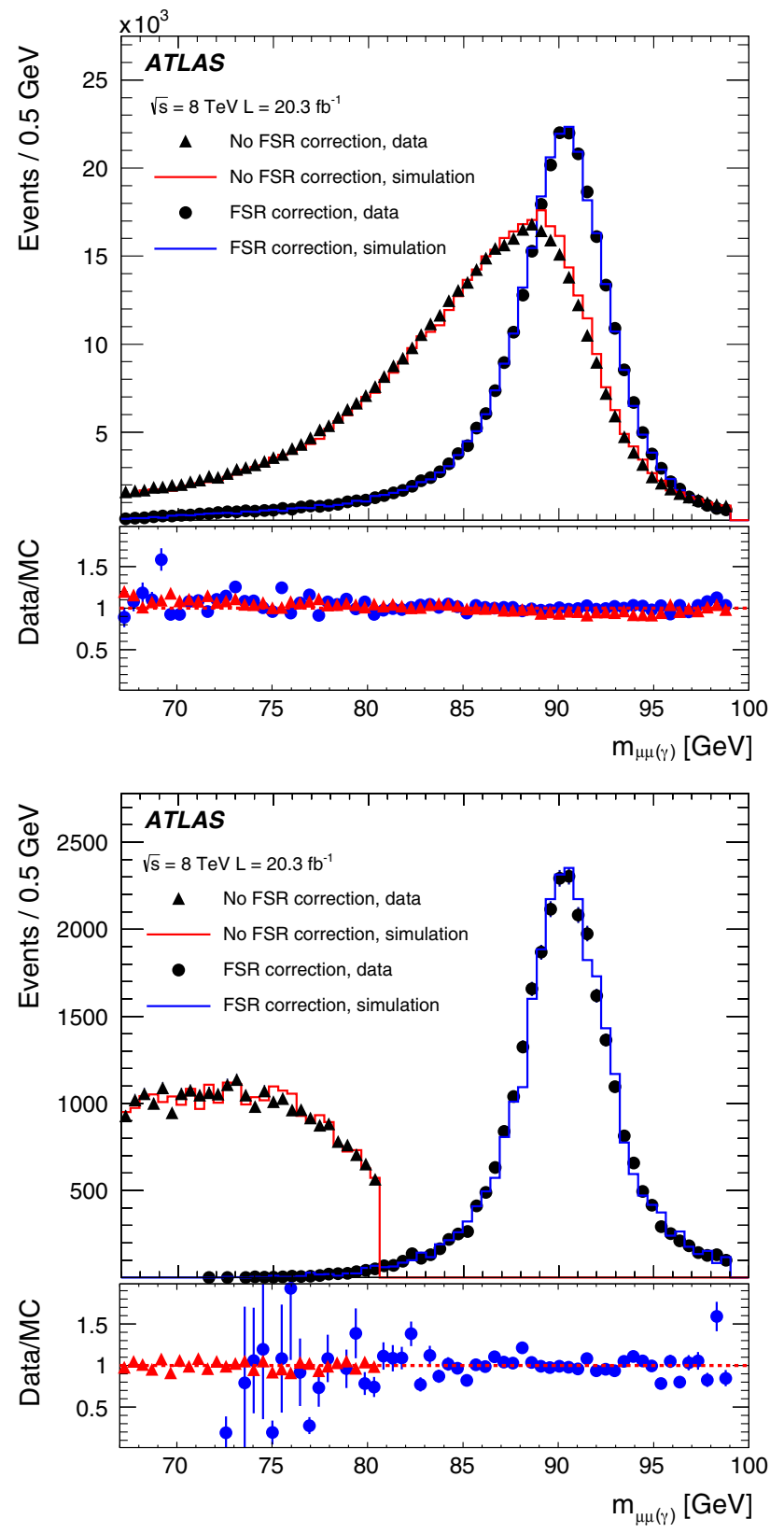

Fig. 17 Invariant mass distribution of $Z \rightarrow \mu \mu$ events with identified FSR in data before (filled triangles) and after (filled circles) FSR correction, for collinear (top) and non-collinear (bottom) FSR. The MC prediction is shown before correction (red histogram) and after correction (blue histogram)

MC studies, the collinear FSR selection has an efficiency of $70 \pm 4 \%$ for FSR photons emitted with $E_{T}>1.5 \mathrm{GeV}$ and $\Delta R_{\gamma, \mu}<0.15$ in the fiducial region defined requiring $|\eta|<2.37$ and excluding the calorimeter crack region $1.37<$ $|\eta|<1.52$. About $85 \%$ of the corrected events have genuine FSR photons, with the remaining photons coming from muon bremsstrahlung or ionization or from random matching with energy depositions from other sources. The fraction of all $Z \rightarrow \mu \mu$ events corrected with a collinear FSR photon is $\simeq 4 \%$. The non-collinear FSR selection has an efficiency of $60 \pm 3 \%$ in the fiducial region and a purity of $\geq 95 \%$. The fraction of $Z \rightarrow \mu \mu$ events corrected with a non-collinear FSR photon is $\simeq 1 \%$.

The FSR correction may introduce systematic variations in the invariant mass scale and resolution. To study these effects, a Gaussian fit of the $Z \rightarrow \mu \mu$ distribution has been performed in the mass range $91.18 \pm 3.00 \mathrm{GeV}$. The FSR correction induces a mass shift of $+40 \pm 3 \mathrm{MeV}$ and an improvement of the resolution of $3 \pm 1 \%$ in the full $Z \rightarrow \mu \mu$ sample. The effects observed in the data are well reproduced by the MC. The systematic uncertainty introduced by the FSR recovery on the inclusive $Z$ mass scale can be understood by considering a $0.5 \%$ photon energy scale uncertainty, the fact that only $5 \%$ of the $Z$ events are corrected, and that the fraction of energy carried by the photons is a few $\%$. This leads to a systematic uncertainty smaller than $2 \mathrm{MeV}$.

The effect of pile up on the FSR correction has been estimated by dividing the data and the MC into three categories based on the average number of interactions per bunch crossing: $\langle\mu\rangle=0-17,17-23,23-40$. A comparison of the fitted $Z$ mass between data and $\mathrm{MC}$ has been performed in the three categories and no dependence on $\langle\mu\rangle$ was observed. Good agreement between data and MC within the statistical uncertainties was found.

\section{Conclusions}

The performance of the ATLAS muon reconstruction has been measured using data from LHC $p p$ collisions at $\sqrt{s}=$ $7-8 \mathrm{TeV}$. The muon reconstruction efficiency is close to $99 \%$ over most of the pseudorapidity range of $|\eta|<2.5$ and for $p_{\mathrm{T}}>10 \mathrm{GeV}$. The large collected sample of $9 \mathrm{M} Z \rightarrow \mu \mu$ decays allows the measurement of the efficiency over the full acceptance of $|\eta|<2.7$, and with a precision at the 1 per-mille level for $|\eta|<2.5$. By including $J / \psi \rightarrow \mu \mu$ decays, the efficiency measurement has been extended over the transverse momentum range from $p_{\mathrm{T}} \approx 4 \mathrm{GeV}$ to $p_{\mathrm{T}} \approx$ $100 \mathrm{GeV}$.

The muon momentum scale and resolution has been studied in detail using large calibration samples of $J / \psi \rightarrow \mu \mu$, $\Upsilon \rightarrow \mu \mu$ and $Z \rightarrow \mu \mu$ decays. These studies have been used to correct the MC simulation to improve the data-MC agreement and to minimize the uncertainties in physics analyses. The momentum scale for combined muons is known with an uncertainty of $\pm 0.05 \%$ for $|\eta|<1$, which increases to $\lesssim 0.2 \%$ for $|\eta|>2.3$ for $Z \rightarrow \mu \mu$ events. The dimuon mass resolution is $\approx 1.2 \%(2 \%)$ at low- $p_{\mathrm{T}}$ increasing to $\approx 2 \%(3 \%)$ at $p_{\mathrm{T}} \approx 100 \mathrm{GeV}$ for $|\eta|<1(|\eta|>1)$. The resolution is reproduced by the corrected simulation within relative uncertainties of $3 \%$ to $10 \%$ depending on $\eta$ and $p_{\mathrm{T}}$. 
The mass resolution for the $Z \rightarrow \mu \mu$ resonance was found to improve when photons from QED final state radiation are recovered. The FSR recovery allows to recover $\approx 4 \%$ of the events from the low-mass tail to the peak region, improving the dimuon mass resolution by $\approx 3 \%$.

Acknowledgments We thank CERN for the very successful operation of the LHC, as well as the support staff from our institutions without whom ATLAS could not be operated efficiently. We acknowledge the support of ANPCyT, Argentina; YerPhI, Armenia; ARC, Australia; BMWF and FWF, Austria; ANAS, Azerbaijan; SSTC, Belarus; CNPq and FAPESP, Brazil; NSERC, NRC and CFI, Canada; CERN; CONICYT, Chile; CAS, MOST and NSFC, China; COLCIENCIAS, Colombia; MSMT CR, MPO CR and VSC CR, Czech Republic; DNRF, DNSRC and Lundbeck Foundation, Denmark; EPLANET, ERC and NSRF, European Union; IN2P3-CNRS, CEA-DSM/IRFU, France; GNSF, Georgia; BMBF, DFG, HGF, MPG and AvH Foundation, Germany; GSRT and NSRF, Greece; ISF, MINERVA, GIF, ICORE and Benoziyo Center, Israel; INFN, Italy; MEXT and JSPS, Japan; CNRST, Morocco; FOM and NWO, The Netherlands; BRF and RCN, Norway; MNiSW and NCN, Poland; GRICES and FCT, Portugal; MNE/IFA, Romania; MES of Russia and ROSATOM, Russian Federation; JINR; MSTD, Serbia; MSSR, Slovakia; ARRS and MIZŠ, Slovenia; DST/NRF, South Africa; MINECO, Spain; SRC and Wallenberg Foundation, Sweden; SER, SNSF and Cantons of Bern and Geneva, Switzerland; NSC, Taiwan; TAEK, Turkey; STFC, the Royal Society and Leverhulme Trust, UK; DOE and NSF, USA. The crucial computing support from all WLCG partners is acknowledged gratefully, in particular from CERN and the ATLAS Tier-1 facilities at TRIUMF (Canada), NDGF (Denmark, Norway, Sweden), CC-IN2P3 (France), KIT/GridKA (Germany), INFN-CNAF (Italy), NL-T1 (The Netherlands), PIC (Spain), ASGC (Taiwan), RAL (UK) and BNL (USA) and in the Tier-2 facilities worldwide.

Open Access This article is distributed under the terms of the Creative Commons Attribution License which permits any use, distribution, and reproduction in any medium, provided the original author(s) and the source are credited.

Funded by $\mathrm{SCOAP}^{3}$ / License Version CC BY 4.0.

\section{Appendix A: Results with different reconstruction "Chains"}

This appendix reports the main results obtained with the other two muon reconstruction software packages used to process 2012 data, Chain 2 and the unified reconstruction programme Chain-3. Figure 18 shows the efficiency as a function of $\eta$ for Chain 2 and Chain 3 and is similar to Fig. 3 for Chain 1.

The efficiency drop that is observed in Chain 1 for CB muons at $|\eta| \simeq 1.2$ is not present in the other two packages due to the less strict selection on the number of measurements in the MS. These relaxed requirements also improve the data/MC agreement. In Chain 2 the $\mathrm{CB}+\mathrm{ST}$ efficiency is higher than the $\mathrm{CB}$ efficiency alone, similarly to Chain 1. For Chain 3, the distinction between CB and ST muons is not applicable anymore since a ID-MS combined momentum fit is performed also in the case of muons that traversed only one MS chamber, a category that is assigned to ST muons in
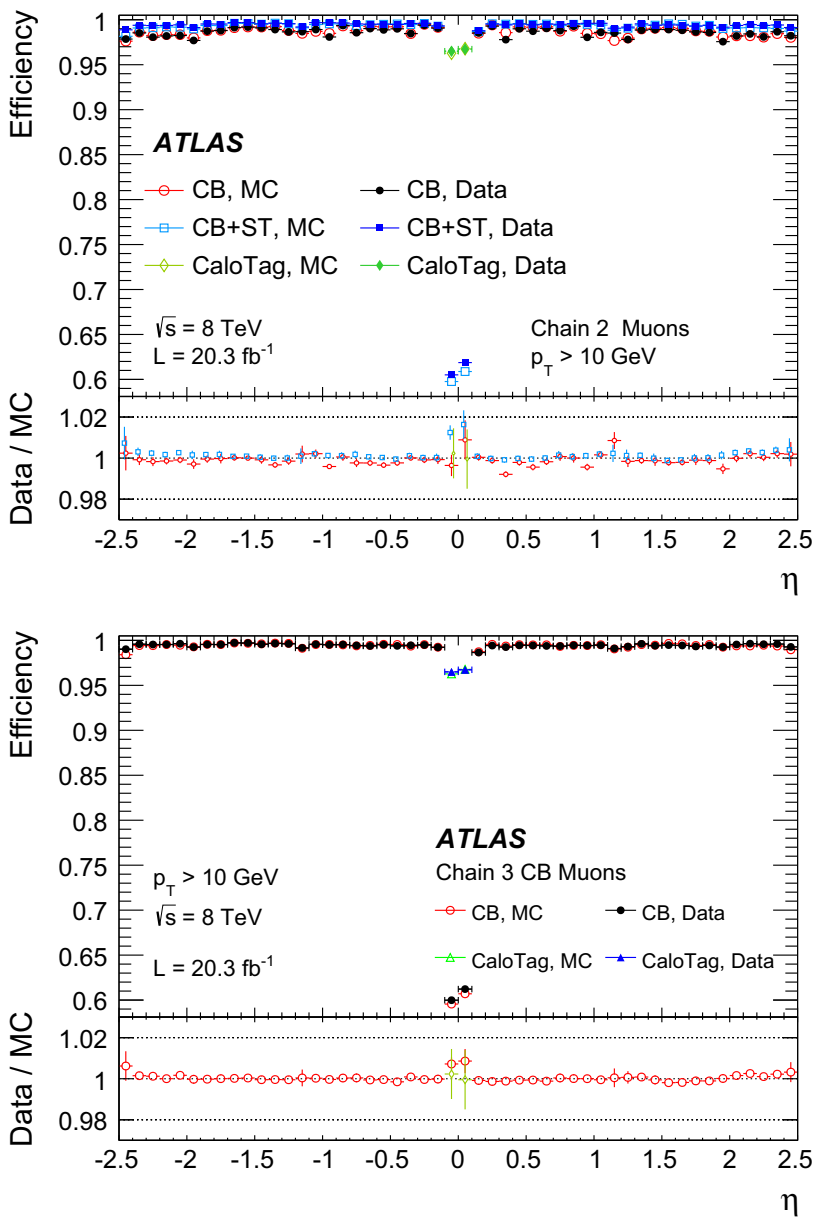

Fig. 18 Muon reconstruction efficiency as a function of $\eta$, measured using $Z \rightarrow \mu \mu$ events, for muons reconstructed with Chain-2 (top) and Chain-3 (bottom), for different muon reconstruction types. CaloTag muons are shown in the region $|\eta|<0.1$, where they are used in physics analyses. The error bars shown for the efficiencies represent the statistical uncertainty. The panel at the bottom shows the ratio between the measured and predicted efficiencies. The error bars show statistical and systematic uncertainties added in quadrature

Chain 1 and (with some exceptions) in Chain 2. Therefore only one type of Chain 3 muons is considered, which was tuned to provide a purity similar to that of the CB muons of Chain 1.

The momentum resolution of the three chains is very similar, with Chain 3 having approximately $2 \%$ better resolution than Chain 1. The data/MC agreement and the amount of correction applied to the simulation is compatible among the three packages.

\section{Appendix B: Results on 2011 data}

During the 2011 data taking period, the LHC delivered $p p$ collisions at a center of mass energy of $\sqrt{s}=7 \mathrm{TeV}$. A sample corresponding to an integrated luminosity of $4.5 \mathrm{fb}^{-1}$ 


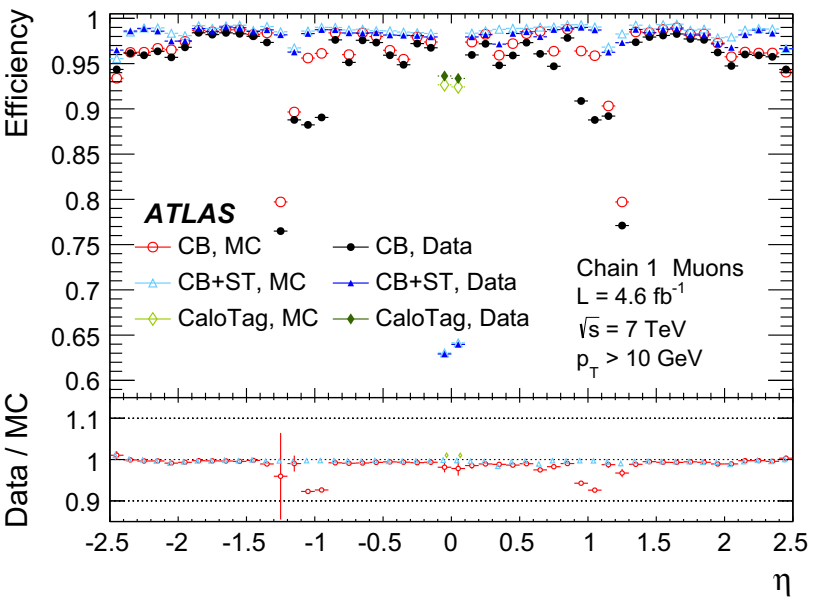

Fig. 19 Muon reconstruction efficiency as a function of $\eta$ measured in $Z \rightarrow \mu \mu$ events in the 2011 data sample for different muon reconstruction types. CaloTag muons are only shown in the region $|\eta|<0.1$, where they are used in physics analyses. For the efficiency, the error bars indicate the statistical uncertainty. The panel at the bottom shows the ratio between the measured and MC efficiencies. The error bars on the ratios show the combination of statistical and systematic uncertainties. The lower efficiency of CB muons at $|\eta| \approx 1.2$ is due to the fact that some of the MS chambers were not yet installed

has been used to measure the muon reconstruction performance with 2011 data. The ID and MS configurations were the same in 2011 as in 2012, with the exception of additional MDT chambers installed between the two periods to increase the number of MS layers from one to two at $\eta=-1.2$ and in part of the region at $\eta=1.2$. The trigger thresholds were in general lower in 2011. The reconstruction programs used for 2011 data were similar to those used in 2012, although several improvements have been introduced between the two periods. Tighter requirements on the ID tracks associated to the muon track were applied in 2011. Similar MC samples as those used for the study of 2012 data have been generated at $\sqrt{s}=7 \mathrm{TeV}$ for the study of muon performance in 2011, using the same simulation based on GEANT4. The reconstruction of the 2011 simulated data was performed with ideal alignment in the MS.

The efficiency, calculated with the "tag and probe" method as in 2012, is presented in Fig. 19 for Chain 1 muons. The main difference with respect to 2012 is the lower efficiency of CB muons at $|\eta| \simeq 1.2$, in which a layer of MDT chambers was missing, and the inefficiency introduced by the tighter ID selection.

The momentum corrections have been derived for the 2011 MC in the same way as for the $2012 \mathrm{MC}$. After correction, the mass scales of data and MC are in good agreement as shown in Fig. 20. Due to the smaller data sample, the momentum corrections have larger uncertainties than in 2012. The resolution for $\mathrm{CB}$ muons obtained with $Z$ events is presented in Fig. 21. The resolution of the uncorrected MC is $\approx 20 \%$

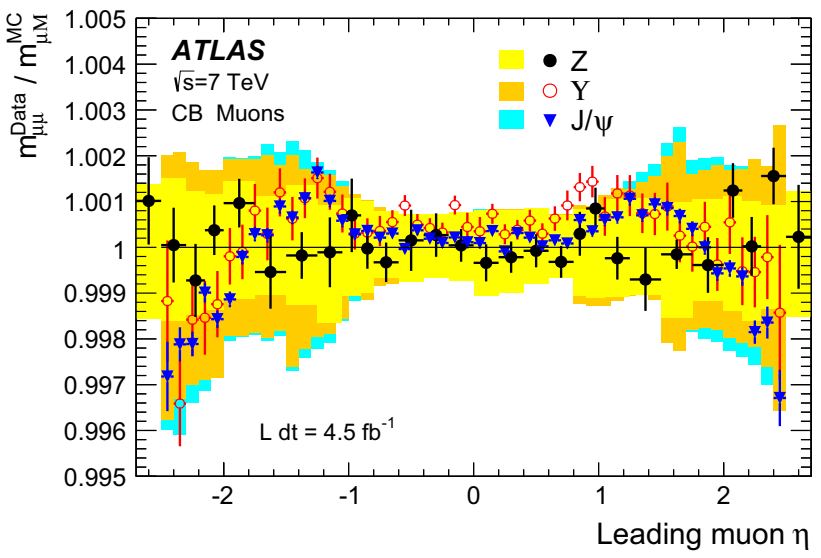

Fig. 20 Ratio of the fitted mean mass, $\left\langle m_{\mu \mu}\right\rangle$, for data and corrected MC in the 2011 data samples. Measurements from $J / \psi, \Upsilon$ and $Z$ events are shown as a function of $\eta$ of the highest- $p_{\mathrm{T}}$ muon. The bands show the uncertainty on the MC corrections extracted for the three calibration samples

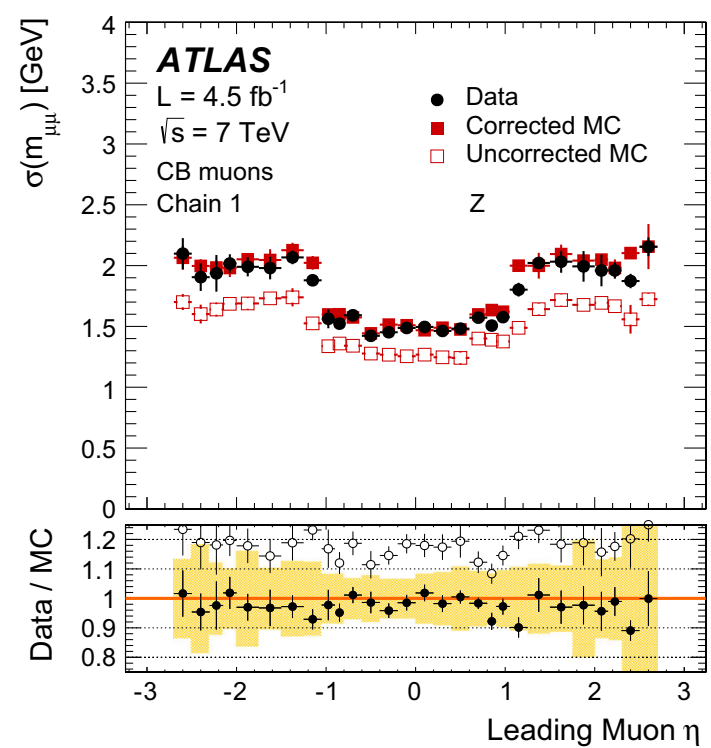

Fig. 21 Dimuon mass resolution $\sigma\left(m_{\mu \mu}\right)$ reconstructed with Chain 1 $\mathrm{CB}$ muons for $Z \rightarrow \mu \mu$ events recorded in 2011 for data and for uncorrected and corrected $\mathrm{MC}$, as a function of the pseudorapidity of the highest- $p_{\mathrm{T}}$ muon. The lower panel shows the data/MC ratio and the band shows the systematic uncertainty from the momentum corrections

smaller than data, significantly worse than in the 2012 case. This is due to the improvements introduced in the reconstruction of 2012 data, including a better knowledge of the ID and MS alignments, and to the use of the ideal MS alignment in the 2011 simulation.

\section{References}

1. ATLAS Collaboration, JINST 3, S08003 (2008)

2. ATLAS Collaboration, Phys. Rev. D 85, 072004 (2012). arXiv:1109.5141 [hep-ex] 
3. ATLAS Collaboration, JHEP 1405, 068 (2014). arXiv:1402.6263 [hep-ex]

4. ATLAS Collaboration, JHEP 1406, 112 (2014). arXiv:1404.1212 [hep-ex]

5. ATLAS Collaboration, Phys. Lett. B 716, 1-29 (2012). arXiv:1207.7214 [hep-ex]

6. ATLAS Collaboration, Phys. Rev. D 90, 052004 (2014). arXiv: 1406.3827 [hep-ex]

7. ATLAS Collaboration, Phys. Rev. D 87, 015010 (2013). arXiv:1211.1150 [hep-ex]

8. ATLAS Collaboration, Phys. Rev. D 88, 072001 (2013). arXiv:1308.4075 [hep-ex]

9. ATLAS Collaboration, Eur. Phys. J. C 74, 3034 (2014). arXiv: 1404.4562 [hep-ex]

10. ATLAS Collaboration, Expected performance of the ATLAS experiment-detector, trigger and physics. CERN, Geneva (2009). arXiv:0901.0512 [hep-ex]

11. ATLAS Collaboration, Eur. Phys. J. C 72, 1849 (2012). arXiv: 1110.1530 [hep-ex]

12. ATLAS Collaboration, submitted to Eur. Phys. J. C (2014). arXiv: 1408.3179 [hep-ex]

13. S. Alioli, P. Nason, C. Oleari, E. Re, JHEP 1006, 043 (2010). arXiv:1002.2581 [hep-ph]

14. T. Sjöstrand, S. Mrenna, P. Skands, Comput. Phys. Commun. 178, 852 (2008). arXiv:0710.3820 [hep-ph]

15. H.-L. Lai et al., Phys. Rev. D 82, 074024 (2010). arXiv:1007.2241 [hep-ph]

16. P. Golonka, Z. Was, Eur. Phys. J. C 45, 97 (2006). arXiv:0506026 [hep-ph]
17. M. L. Mangano et al., JHEP 0307, 001 (2003). arXiv:0206293 [hep-ph]

18. T. Sjöstrand, S. Mrenna, P. Skands, JHEP 0605, 026 (2006). arXiv:0603175 [hep-ph]

19. ATLAS Collaboration, Phys. Rev. D 85, 092002 (2012). arXiv:1201.1276 [hep-ex]

20. T. Gleisberg et al., JHEP 0902, 007 (2009). arXiv:0811.4622 [hep$\mathrm{ph}]$

21. S. Frixione, B. R. Webber, JHEP 0206, 029 (2002). arXiv:0204244 [hep-ph]

22. ATLAS Collaboration, Eur. Phys. J. C 70, 823 (2010). arXiv: 1005.4568 [physics.ins-det]

23. S. Agostinelli et al., Nucl. Instrum. Method A 506, 250 (2003)

24. A. Martin, W. Stirling, R. Thorne, G. Watt, Eur. Phys. J. C 63, 189 (2009). arXiv:0901.0002 [hep-ph]

25. C. Grupen, B. Shwartz, Particle Detectors, 2nd edn. Cambridge University Press, Cambridge (2008)

26. M. Oreglia, SLAC-R-0236 (1980) (appendix D)

27. F. James, M. Roos, Comput. Phys. Commun. 10, 343-367 (1975)

28. Particle Data Group, J. Beringer et al. Phys. Rev. D 86, 010001 (2012)

29. ATLAS Collaboration, Phys. Lett. B 732, 8-27 (2014). arXiv:1402.3051 [hep-ex]

30. ATLAS Collaboration, Phys. Rev. D 89, 052004 (2014). arXiv:1311.1440 [hep-ex]

\section{ATLAS Collaboration}

G. Aad $^{84}$, B. Abbott ${ }^{112}$, J. Abdallah ${ }^{152}$, S. Abdel Khalek ${ }^{116}$, O. Abdinov ${ }^{11}$, R. Aben ${ }^{106}$, B. Abi ${ }^{113}$, M. Abolins ${ }^{89}$, $\begin{array}{lll}\text { O. S. AbouZeid } & 159\end{array}$ H. Abramowicz ${ }^{154}$, H. Abreu ${ }^{153}, \quad$ R. Abreu ${ }^{30}, \quad$ Y. Abulaiti147a,147b , B. S. Acharya ${ }^{165 a, 165 b, a}$,

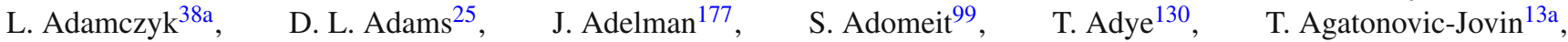
J. A. Aguilar-Saavedra ${ }^{125 a, 125 f}$, M. Agustoni ${ }^{17}$, S. P. Ahlen ${ }^{22}$, F. Ahmadov ${ }^{64, b}$, G. Aielli ${ }^{134 a, 134 b}$, H. Akerstedt ${ }^{147 a, 147 b}$,

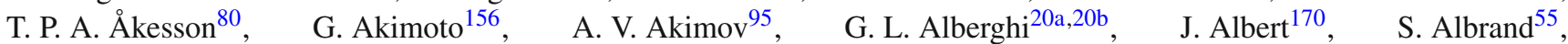

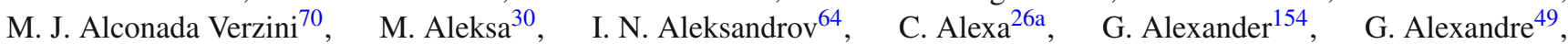
T. Alexopoulos ${ }^{10}$, M. Alhroob ${ }^{165 a, 165 b}$, G. Alimonti ${ }^{90 a}$, L. Alio ${ }^{84}$, J. Alison ${ }^{31}$, B. M. M. Allbrooke ${ }^{18}$, L. J. Allison ${ }^{71}$,

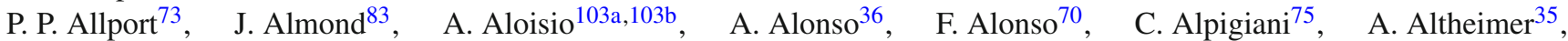
B. Alvarez Gonzalez ${ }^{89}$, M. G. Alviggi ${ }^{103 a, 103 b}$, K. Amako ${ }^{65}$, Y. Amaral Coutinho ${ }^{24 a}$ ， C. Amelung ${ }^{23}$ ， D. Amidei ${ }^{88}$, S. P. Amor Dos Santos ${ }^{125 a, 125 c}$, A. Amorim ${ }^{125 a, 125 b}$, S. Amoroso ${ }^{48}$, N. Amram ${ }^{154}$, G. Amundsen ${ }^{23}$, C. Anastopoulos ${ }^{140}$,

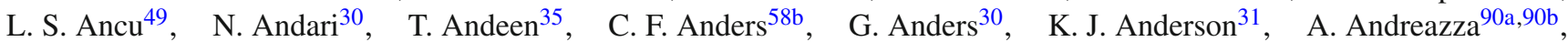
V. Andrei ${ }^{58 a}$, X. S. Anduaga ${ }^{70}$, S. Angelidakis ${ }^{9}$, I. Angelozzi ${ }^{106}, \quad$ P. Anger ${ }^{44}$, A. Angerami ${ }^{35}, \quad$ F. Anghinolfi ${ }^{30}$, A. V. Anisenkov ${ }^{108}$, N. Anjos ${ }^{125 a}$, A. Annovi ${ }^{47}$, A. Antonaki ${ }^{9}$, M. Antonellii ${ }^{47}$ A. Antonov ${ }^{97}$, J. Antos $^{145 b}$, F. Anulli ${ }^{133 a}$, M. Aoki ${ }^{65}$, L. Aperio Bella ${ }^{18}$, R. Apolle ${ }^{119, c}$, G. Arabidze ${ }^{89}$, I. Aracena ${ }^{144}$, Y. Arai ${ }^{65}$, J. P. Araque ${ }^{125 a}$, A. T. H. Arce ${ }^{45}$, J.-F. Arguin ${ }^{94}$, S. Argyropoulos ${ }^{42}$, M. Arik ${ }^{19 a}$, A. J. Armbruster ${ }^{30}$, O. $\operatorname{Arnaez}^{30}$, V. Arnal ${ }^{81}$, H. Arnold ${ }^{48}$, M. Arratia ${ }^{28}$, O. Arslan ${ }^{21}$, A. Artamonov ${ }^{96}$, G. Artoni ${ }^{23}$, S. Asai ${ }^{156}$, N. Asbah ${ }^{42}$, A. Ashkenazi ${ }^{154}$, B. Åsman 147a,147b, L. Asquith ${ }^{6}$, K. Assamagan ${ }^{25}$, R. Astalos ${ }^{145 a}$, M. Atkinson ${ }^{166}$, N. B. Atlay ${ }^{142}$, B. Auerbach ${ }^{6}$, K. Augsten ${ }^{127}$, M. Aurousseau ${ }^{146 b}$, G. Avolio $^{30}$, G. Azuelos ${ }^{94, d}$, Y. Azuma ${ }^{156}$, M. A. Baak ${ }^{30}$, A. Baas ${ }^{58 a}$, C. Bacci ${ }^{135 a, 135 b}$, H. Bachacou ${ }^{137}$, K. Bachas ${ }^{155}$, M. Backes ${ }^{30}$, M. Backhaus ${ }^{30}$, J. Backus Mayes ${ }^{144}$, E. Badescu' ${ }^{26 a}$, P. Bagiacchi ${ }^{133 a, 133 b}$, P. Bagnaia ${ }^{133 a, 133 b}$, Y. Bai ${ }^{33 a}$, T. Bain ${ }^{35}$, J. T. Baines ${ }^{130}$, O. K. Baker ${ }^{177}$, P. Balek ${ }^{128}$, F. Balli ${ }^{137}$, E. Banas ${ }^{39}$, Sw. Banerjee ${ }^{174}$, A. A. E. Bannoura ${ }^{176}$, V. Bansal ${ }^{170}$, H. S. Bansil ${ }^{18}$, L. Barak ${ }^{173}$, S. P. Baranov95, E. L. Barberio ${ }^{87}$, D. Barberis ${ }^{50 a, 50 b}$, M. Barbero ${ }^{84}$, T. Barillari ${ }^{100}$, M. Barisonzi

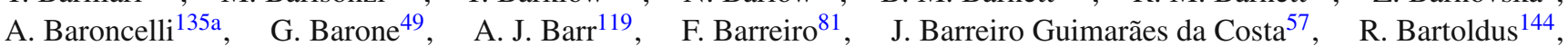
A. E. Barton ${ }^{71}$, P. Bartos ${ }^{145 a}$, V. Bartsch ${ }^{150}$, A. Bassalat ${ }^{116}$, A. Basye ${ }^{166}$, R. L. Bates ${ }^{53}$, J. R. Batley ${ }^{28}$, M. Battaglia ${ }^{138}$, M. Battistin ${ }^{30}$, F. Bauer ${ }^{137}$, H. S. Bawa ${ }^{144, e}$, M. D. Beattie ${ }^{71}$, T. Beau ${ }^{79}$, P. H. Beauchemin ${ }^{162}$, R. Beccherle ${ }^{123 a, 123 b}$, 
P. Bechtle ${ }^{21}$, H. P. Beck ${ }^{17}$, K. Becker ${ }^{176}$, S. Becker ${ }^{99}$, M. Beckingham ${ }^{171}$, C. Becot ${ }^{116}$, A. J. Beddall ${ }^{19 c}$, A. Beddall ${ }^{19 c}$, S. Bedikian ${ }^{177}$ ， V. A. Bednyakov ${ }^{64}$, C. P. Bee ${ }^{149}$ ， L. J. Beemster ${ }^{106}$, T. A. Beermann ${ }^{176}$, M. Begel ${ }^{25}$, K. Behr ${ }^{119}$, C. Belanger-Champagne ${ }^{86}$, P. J. Bell ${ }^{49}$ ，W. H. Bell ${ }^{49}$ ， G. Bella ${ }^{154}$ ， L. Bellagamba ${ }^{20 a}$ ，A. Bellerive ${ }^{29}$ ， M. Bellomo ${ }^{85}$, K. Belotskiy ${ }^{97}$, O. Beltramello ${ }^{30}$, O. Benary ${ }^{154}$, D. Benchekroun ${ }^{136 a}$, K. Bendtz ${ }^{147 a, 147 b}$, N. Benekos ${ }^{166}$, Y. Benhammou ${ }^{154}$, E. Benhar Noccioli ${ }^{49}$, J. A. Benitez Garcia ${ }^{160 b}$, D. P. Benjamin ${ }^{45}$, J. R. Bensinger ${ }^{23}$, K. Benslama ${ }^{131}$, S. Bentvelsen ${ }^{106}$,

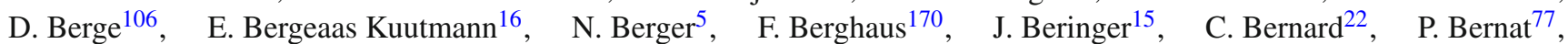
C. Bernius ${ }^{78}$, F. U. Bernlochner ${ }^{170}$, T. Berry ${ }^{76}$, P. Berta ${ }^{128}$, C. Bertella ${ }^{84}$, G. Bertoli ${ }^{147 a, 147 b}$, F. Bertolucci ${ }^{123 a, 123 b}$, C. Bertsche ${ }^{112}$, D. Bertsche ${ }^{112}$ ，M. Bessner ${ }^{42}$, M. I. Besana90a, G. J. Besjes ${ }^{105}$ ，O. Bessidskaia ${ }^{147 a, 147 b}$ ，N. Besson ${ }^{137}$, C. Betancourt ${ }^{48}$, S. Bethke ${ }^{100}$, W. Bhimji ${ }^{46}$, R. M. Bianchi ${ }^{124}$, L. Bianchini ${ }^{23}$, M. Bianco ${ }^{30}$, O. Biebel ${ }^{99}$, S. P. Bieniek ${ }^{77}$, K. Bierwagen ${ }^{54}$, J. Biesiada ${ }^{15}$, M. Biglietti ${ }^{135 a}$, J. Bilbao De Mendizabal ${ }^{49}$, H. Bilokon ${ }^{47}$, M. Bindi ${ }^{54}$, S. Binet ${ }^{116}$,

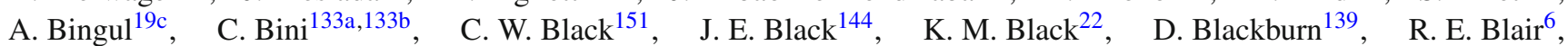
J.-B. Blanchard ${ }^{137}$, T. Blazek ${ }^{145 a}$, I. Bloch ${ }^{42}$, C. Blocker $^{23}$ ，W. Blum ${ }^{82, *}$, U. Blumenschein ${ }^{54}$, G. J. Bobbink ${ }^{106}$,

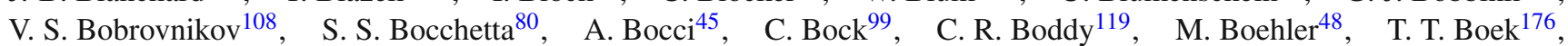
J. A. Bogaerts ${ }^{30}$, A. G. Bogdanchikov ${ }^{108}$, A. Bogouch ${ }^{91,{ }^{*}}$, C. Bohm ${ }^{147 a}$, J. Bohm ${ }^{126}$, V. Boisvert ${ }^{76}$, T. Bold ${ }^{38 a}$, V. Boldea ${ }^{26 a}$, A. S. Boldyrev ${ }^{98}$, M. Bomben ${ }^{79}$, M. Bona ${ }^{75}$, M. Boonekamp ${ }^{137}$, A. Borisov ${ }^{129}$, G. Borissov ${ }^{71}$, M. Borri ${ }^{83}$, S. Borroni ${ }^{42}$, J. Bortfeldt ${ }^{99}$, V. Bortolotto ${ }^{135 a, 135 b}$, K. Bos ${ }^{106}$, D. Boscherini ${ }^{20 a}$, M. Bosman ${ }^{12}$, H. Boterenbrood ${ }^{106}$, J. Boudreau ${ }^{124}$, J. Bouffard ${ }^{2}$, E. V. Bouhova-Thacker ${ }^{71}$, D. Boumediene ${ }^{34}$, C. Bourdarios ${ }^{116}$, N. Bousson ${ }^{113}$, S. Boutouil ${ }^{136 d}$, A. Boveia ${ }^{31}$, J. Boyd ${ }^{30}$, I. R. Boyko ${ }^{64}$, J. Bracinik ${ }^{18}$, A. Brandt ${ }^{8}$, G. Brandt ${ }^{15}$, O. Brandt ${ }^{58 a}$, U. Bratzler ${ }^{157}$, B. Brau ${ }^{85}$, J. E. Brau ${ }^{115}$, H. M. Braun ${ }^{176, *}$, S. F. Brazzale ${ }^{165 a, 165 c}$, B. Brelier ${ }^{159}$, K. Brendlinger ${ }^{121}$, A. J. Brennan ${ }^{87}$, R. Brenner ${ }^{167}$, S. Bressler ${ }^{173}$, K. Bristow ${ }^{146 c}$, T. M. Bristow ${ }^{46}$, D. Britton ${ }^{53}$, F. M. Brochu ${ }^{28}$, I. Brock ${ }^{21}$, R. Brock ${ }^{89}$, C. Bromberg ${ }^{89}$, J. Bronner ${ }^{100}$, G. Brooijmans ${ }^{35}$, T. Brooks ${ }^{76}$, W. K. Brooks ${ }^{32 b}$, J. Brosamer ${ }^{15}$, E. Brost ${ }^{115}$, J. Brown ${ }^{55}$, P. A. Bruckman de Renstrom ${ }^{39}$, D. Bruncko ${ }^{145 b}$, R. Bruneliere ${ }^{48}$, S. Brunet ${ }^{60}$, A. Bruni ${ }^{20 a}$, G. Bruni ${ }^{20 a}$, M. Bruschi ${ }^{20 a}$, L. Bryngemark ${ }^{80}$, T. Buanes ${ }^{14}$, Q. Buat ${ }^{143}$, F. Bucci ${ }^{49}$, P. Buchholz ${ }^{142}$, R. M. Buckingham ${ }^{119}$, A. G. Buckley ${ }^{53}$, S. I. Buda ${ }^{26 a}$, I. A. Budagov ${ }^{64}$, F. Buehrer ${ }^{48}$, L. Bugge ${ }^{118}$, M. K. Bugge ${ }^{118}$, O. Bulekov 97 , A. C. Bundock ${ }^{73}$, H. Burckhart $^{30}$, S. Burdin ${ }^{73}$, B. Burghgrave ${ }^{107}$, S. Burke ${ }^{130}$, I. Burmeister ${ }^{43}$, E. Busato ${ }^{34}$, D. Büscher ${ }^{48}$, V. Büscher ${ }^{82}$, P. Bussey ${ }^{53}$, C. P. Buszello ${ }^{167}$, B. Butler ${ }^{57}$, J. M. Butler ${ }^{22}$, A. I. Butt ${ }^{3}$, C. M. Buttar ${ }^{53}$, J. M. Butterworth ${ }^{77}$, P. Butti ${ }^{106}$, W. Buttinger ${ }^{28}$, A. Buzatu ${ }^{53}$, M. Byszewski ${ }^{10}$, S. Cabrera Urbán ${ }^{168}$, D. Caforio ${ }^{20 a, 20 b}$, O. Cakir ${ }^{4 a}$, P. Calafiura ${ }^{15}$, A. Calandri ${ }^{137}$, G. Calderini ${ }^{79}$, P. Calfayan ${ }^{99}$, R. Calkins ${ }^{107}$, L. P. Caloba ${ }^{24 a}$, D. Calvet ${ }^{34}, \quad$ S. Calvet ${ }^{34}$, R. Camacho Toro ${ }^{49}, \quad$ S. Camarda ${ }^{42}$, D. Cameron ${ }^{118}$, L. M. Caminada ${ }^{15}$, R. Caminal Armadans ${ }^{12}$, S. Campana ${ }^{30}$, M. Campanellii ${ }^{77}$, A. Campoverde ${ }^{149}$, V. Canale ${ }^{103 a, 103 b}$, A. Canepa ${ }^{160 a}$, M. Cano Bret ${ }^{75}$, J. Cantero ${ }^{81}$, R. Cantrill ${ }^{125 a}$, T. Cao ${ }^{40}$, M. D. M. Capeans Garrido ${ }^{30}$, I. Caprini ${ }^{26 a}$, M. Caprini ${ }^{26 a}$, M. Capua ${ }^{37 a, 37 b}$, R. Caputo ${ }^{82}$, R. Cardarelli ${ }^{134 a}$, T. Carli ${ }^{30}$, G. Carlino ${ }^{103 a}$, L. Carminati ${ }^{90 a}$,90b , S. Caron ${ }^{105}$, E. Carquin ${ }^{32 a}$, G. D. Carrillo-Montoya ${ }^{146 c}$, J. R. Carter $^{28}$, J. Carvalho ${ }^{125 a, 125 c}$, D. Casadei ${ }^{77}$, M. P. Casado ${ }^{12}$, M. Casolino ${ }^{12}$, E. Castaneda-Miranda ${ }^{146 b}$ ， A. Castelli ${ }^{106}$, V. Castillo Gimenez ${ }^{168}$, N. F. Castro ${ }^{125 a}$, P. Catastini ${ }^{57}$, A. Catinaccio ${ }^{30}$, J. R. Catmore ${ }^{118}$, A. Cattai ${ }^{30}$, G. Cattani ${ }^{134 a, 134 b}$, S. Caughron ${ }^{89}$, V. Cavaliere ${ }^{166}$, D. Cavalli ${ }^{90 a}$, M. Cavalli-Sforza ${ }^{12}$, V. Cavasinni ${ }^{123 a, 123 c}$, F. Ceradini ${ }^{135 a, 135 b}$, B. Cerio ${ }^{45}$, K. Cerny ${ }^{128}$, A. S. Cerqueira ${ }^{24 b}$, A. Cerri ${ }^{150}$, L. Cerrito ${ }^{75}$, F. Cerutti ${ }^{15}$, M. Cerv $^{30}$, A. Cervelli ${ }^{17}$, S. A. Cetin ${ }^{19 b}$, A. Chafaq ${ }^{136 a}$, D. Chakraborty ${ }^{107}$, I. Chalupkova ${ }^{128}$, P. Chang ${ }^{166}$, B. Chapleau ${ }^{86}$, J. D. Chapman ${ }^{28}$, D. Charfeddine ${ }^{116}$, D. G. Charlton ${ }^{18}, \quad$ C. C. Chau ${ }^{159}, \quad$ C. A. Chavez Barajas ${ }^{150}, \quad$ S. Cheatham ${ }^{86}$, A. Chegwidden ${ }^{89}$, S. Chekanov ${ }^{6}$, S. V. Chekulaev ${ }^{160 a}$, G. A. Chelkov64,f, M. A. Chelstowska ${ }^{88}$, C. Chen ${ }^{63}$, H. Chen ${ }^{25}$, K. Chen ${ }^{149}$, L. Chen ${ }^{33 \mathrm{~d}, \mathrm{~g}}$, S. Chen ${ }^{33 \mathrm{c}}$, X. Chen ${ }^{146 c}$, Y. Chen ${ }^{66}$, Y. Chen ${ }^{35}$, H. C. Cheng ${ }^{88}$, Y. Cheng ${ }^{31}$, A. Cheplakov ${ }^{64}$, R. Cherkaoui El Moursli ${ }^{136 e}$, V. Chernyatin ${ }^{25, *}$, E. Cheu ${ }^{7}, \quad$ L. Chevalier ${ }^{137}$, V. Chiarella ${ }^{47}$, G. Chiefari ${ }^{103 a, 103 b}$, J. T. Childers ${ }^{6}$, A. Chilingarov ${ }^{71}$, G. Chiodini ${ }^{72 a}$, A. S. Chisholm ${ }^{18}$, R. T. Chislett ${ }^{77}$, A. Chitan ${ }^{26 a}$, M. V. Chizhov ${ }^{64}$, $\begin{array}{lll}\text { S. Chouridou } & \\ & \end{array}$ B. K. B. Chow ${ }^{99}$, D. Chromek-Burckhart ${ }^{30}$, M. L. Chu ${ }^{152}$, J. Chudoba ${ }^{126}$, J. J. Chwastowski ${ }^{39}$, L. Chytka ${ }^{114}$, G. Ciapetti ${ }^{133 a, 133 b}$, A. K. Ciftcia ${ }^{4 a}$ R. Ciftci ${ }^{4 a}$, D. Cinca ${ }^{53}$, V. Cindro ${ }^{74}$, A. Ciocio ${ }^{15}$, P. Cirkovic ${ }^{13 b}$, Z. H. Citron ${ }^{173}$, M. Citterio ${ }^{90 a}$, M. Ciubancan ${ }^{26 a}$, A. Clark ${ }^{49}$, P. J. Clark ${ }^{46}$, R. N. Clarke ${ }^{15}$, W. Cleland ${ }^{124}$, J. C. Clemens ${ }^{84}$, C. Clement ${ }^{147 a, 147 b}$, Y. Coadou ${ }^{84}, \quad$ M. Cobal ${ }^{165 a, 165 c}$, A. Coccaro ${ }^{139}$, J. Cochran ${ }^{63}$, L. Coffey ${ }^{23}$, J. G. Cogan ${ }^{144}$, J. Coggeshall ${ }^{166}$, B. Cole ${ }^{35}$, S. Cole ${ }^{107}$, A. P. Colijn ${ }^{106}$, J. Collot $^{55}$, T. Colombo ${ }^{58 c}$, G. Colon ${ }^{85}$, G. Compostella ${ }^{100}$, P. Conde Muiño ${ }^{125 a, 125 b}$, E. Coniavitis ${ }^{48}$, M. C. Conidi ${ }^{12}$, S. H. Connell ${ }^{146 b}$, I. A. Connelly ${ }^{76}$, S. M. Consonni ${ }^{90 a, 90 b}$, V. Consorti ${ }^{48}$, S. Constantinescu ${ }^{26 a}$, C. Conta ${ }^{120 a, 120 b}$, G. Conti ${ }^{57}$, F. Conventi ${ }^{103 a, h}$, M. Cooke ${ }^{15}$, B. D. Cooper ${ }^{77}$, A. M. Cooper-Sarkar ${ }^{119}$, N. J. Cooper-Smith ${ }^{76}, \quad$ K. Copic ${ }^{15}, \quad$ T. Cornelissen ${ }^{176}, \quad$ M. Corradi ${ }^{20 a}, \quad$ F. Corriveau ${ }^{86, i}$, A. Corso-Radu ${ }^{164}$, A. Cortes-Gonzalez ${ }^{12}$ ， G. Cortiana ${ }^{100}$, G. Costa ${ }^{90 a}$ ， M. J. Costa ${ }^{168}$, D. Costanzo ${ }^{140}$, D. Côté ${ }^{8}$, G. Cottin ${ }^{28}$, G. Cowan $^{76}$, B. E. Cox ${ }^{83}$, K. Cranmer ${ }^{109}$, G. Cree ${ }^{29}$, S. Crépé-Renaudin ${ }^{55}$, F. Crescioli ${ }^{79}$, W. A. Cribbs ${ }^{147 a, 147 b}$, M. Crispin Ortuzar ${ }^{119}$, M. Cristinziani ${ }^{21}$, V. Croft ${ }^{105}$, G. Crosetti ${ }^{37,37 b}$, C.-M. Cuciuc ${ }^{26 a}$, T. Cuhadar Donszelmann ${ }^{140}$,

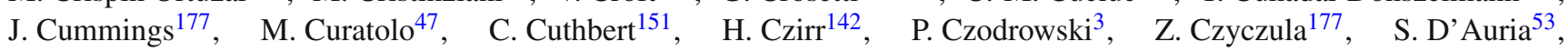


M. D’Onofrio ${ }^{73}$ ， M. J. Da Cunha Sargedas De Sousa ${ }^{125 a, 125 b}$ ， C. Da Via ${ }^{83}$ ，W. Dabrowski ${ }^{38 a}$ ，A. Dafinca ${ }^{119}$ ， T. Dai ${ }^{88}$, O. Dale ${ }^{14}$, F. Dallaire ${ }^{94}$, C. Dallapiccola ${ }^{85}$, M. Dam ${ }^{36}$, A. C. Daniells ${ }^{18}$, M. Dano Hoffmann ${ }^{137}$, V. Dao ${ }^{48}$, G. Darbo ${ }^{50 a}$, S. Darmora ${ }^{8}$, J. A. Dassoulas ${ }^{42}$, A. Dattagupta ${ }^{60}$, W. Davey ${ }^{21}$, C. David ${ }^{170}$, T. Davidek ${ }^{128}$, E. Davies ${ }^{119, c}$, M. Davies ${ }^{154}$, O. Davignon ${ }^{79}$, A. R. Davison ${ }^{77}$, P. Davison ${ }^{77}$, Y. Davygora ${ }^{58 a}$, E. Dawe ${ }^{143}$, I. Dawson ${ }^{140}$, R. K. Daya-Ishmukhametova ${ }^{85}$, K. De ${ }^{8}$, R. de Asmundis ${ }^{103 a}$, S. De Castro ${ }^{20 a, 20 b}$, S. De Cecco ${ }^{79}$, N. De Groot ${ }^{105}$, P. de Jong ${ }^{106}$, H. De la Torre ${ }^{81}$,

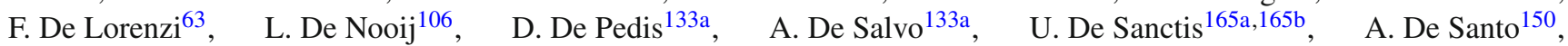
J. B. De Vivie De Regie ${ }^{116}$ ， W. J. Dearnaley ${ }^{71}$ ， R. Debbe ${ }^{25}$ ， C. Debenedetti ${ }^{138}$ ， B. Dechenaux ${ }^{55}$ ， D. V. Dedovich ${ }^{64}$, I. Deigaard ${ }^{106}$, J. Del Peso ${ }^{81}$, T. Del Prete ${ }^{123 a, 123 b}$, F. Deliot ${ }^{137}$, C. M. Delitzsch ${ }^{49}$, M. Deliyergiyev ${ }^{74}$, A. Dell' Acqua ${ }^{30}$, L. Dell'Asta ${ }^{22}$, M. Dell'Orso ${ }^{123 a, 123 b}$, M. Della Pietra ${ }^{103 a, h}$, D. della Volpe ${ }^{49}$, M. Delmastro ${ }^{5}$, P. A. Delsart ${ }^{55}$, C. Deluca ${ }^{106}$, S. Demers ${ }^{177}$, M. Demichev ${ }^{64}$, A. Demilly ${ }^{79}$ ， S. P. Denisov ${ }^{129}$ ， D. Derendarz ${ }^{39}$ ， J. E. Derkaoui ${ }^{136 d}$, F. Derue ${ }^{79}$, P. Dervan ${ }^{73}$, K. Desch $^{21}$, C. Deterre ${ }^{42}$, P. O. Deviveiros ${ }^{106}$, A. Dewhurst ${ }^{130}$, S. Dhaliwal ${ }^{106}$, A. Di Ciaccio ${ }^{134 a, 134 b}$, L. Di Ciaccio ${ }^{5}$ A. Di Domenico ${ }^{133 a, 133 b}$, C. Di Donato103a,103b, A. Di Girolamo ${ }^{30}$, B. Di Girolamo ${ }^{30}$, A. Di Mattia ${ }^{153}$, B. Di Micco ${ }^{135 a, 135 b}$, R. Di Nardo ${ }^{47}$, A. Di Simone ${ }^{48}$, R. Di Sipio ${ }^{20 a}, 20 b$, D. Di Valentino ${ }^{29}$, F. A. Dias ${ }^{46}$, M. A. Diaz ${ }^{32 a}$, E. B. Diehl ${ }^{88}$, J. Dietrich ${ }^{42}$, T. A. Dietzsch ${ }^{58 a}$, S. Diglio ${ }^{84}$, A. Dimitrievska ${ }^{13 a}$, J. Dingfelder ${ }^{21}$, C. Dionisi ${ }^{133 a, 133 b}$, P. Dita ${ }^{26 a}$, S. Dita ${ }^{26 a}$, F. Dittus ${ }^{30}$, F. Djama ${ }^{84}$, T. Djobava ${ }^{51 b}$, M. A. B. do Vale ${ }^{24 c}$, A. Do Valle Wemans ${ }^{125 a, 125 g}$, T. K. O. Doan ${ }^{5}$, D. Dobos ${ }^{30}$, C. Doglioni ${ }^{49}$, T. Doherty ${ }^{53}$, T. Dohmae ${ }^{156}$, J. Dolejsi ${ }^{128}$, Z. Dolezal ${ }^{128}$, B. A. Dolgoshein ${ }^{97, *}$, M. Donadelli24d, S. Donati ${ }^{123 a, 123 b}$, P. Dondero ${ }^{120 a, 120 b}, \quad$ J. Donini ${ }^{34}$ ， J. Dopke ${ }^{130}, \quad$ A. Doria ${ }^{103 a}, \quad$ M. T. Dova ${ }^{70}$, A. T. Doyle ${ }^{53}$, M. Dris ${ }^{10}$, J. Dubbert ${ }^{88}$, S. Dube ${ }^{15}$, E. Dubreuil ${ }^{34}$, E. Duchovni ${ }^{173}$, G. Duckeck ${ }^{99}$, O. A. Ducu ${ }^{26 a}$, D. Duda ${ }^{176}$, A. Dudarev ${ }^{30}$, F. Dudziak ${ }^{63}$, L. Duflot ${ }^{116}$, L. Duguid ${ }^{76}$, M. Dührssen ${ }^{30}$, M. Dunford ${ }^{58 a}$, H. Duran Yildiz ${ }^{4 a}$, M. Düren ${ }^{52}$, A. Durglishvili51b, M. Dwuznik ${ }^{38 a}$, M. Dyndal ${ }^{38 a}$, J. Ebke ${ }^{99}$, W. Edson ${ }^{2}$, N. C. Edwards ${ }^{46}$, W. Ehrenfeld ${ }^{21}$, T. Eifert ${ }^{144}$, G. Eigen ${ }^{14}$, K. Einsweiler ${ }^{15}$, T. Ekelof ${ }^{167}$, M. El Kacimi ${ }^{136 c}$, M. Ellert ${ }^{167}$, S. Elles ${ }^{5}$, F. Ellinghaus ${ }^{82}$, N. Ellis ${ }^{30}$, J. Elmsheuser ${ }^{99}$, M. Elsing ${ }^{30}$, D. Emeliyanov ${ }^{130}$, Y. Enari ${ }^{156}$, O. C. Endner ${ }^{82}$, M. Endo ${ }^{117}$, R. Engelmann ${ }^{149}$, J. Erdmann ${ }^{177}$, A. Ereditato ${ }^{17}$, D. Eriksson ${ }^{147 a}$, G. Ernis ${ }^{176}$, J. Ernst ${ }^{2}$, M. Ernst ${ }^{25}$, J. Ernwein ${ }^{137}$, D. Errede ${ }^{166}$, S. Errede ${ }^{166}$, E. Ertel ${ }^{82}$, M. Escalier ${ }^{116}$, H. Esch ${ }^{43}$, C. Escobar ${ }^{124}$, B. Esposito ${ }^{47}$, A. I. Etienvre ${ }^{137}$, E. Etzion ${ }^{154}$, H. Evans ${ }^{60}$, A. Ezhilov ${ }^{122}$, L. Fabbri ${ }^{20 a}$,20b, G. Facini ${ }^{31}$, R. M. Fakhrutdinov ${ }^{129}$, S. Falciano ${ }^{133 a}$, R. J. Falla ${ }^{77}$, J. Faltova ${ }^{128}$, Y. Fang ${ }^{33 a}$, M. Fanti ${ }^{90 a}$,90b, A. Farbin ${ }^{8}$, A. Farilla ${ }^{135 a}$, T. Farooque ${ }^{12}$, S. Farrell ${ }^{15}$, S. M. Farrington ${ }^{171}$, P. Farthouat ${ }^{30}$, F. Fassi ${ }^{136 e}$, P. Fassnacht ${ }^{30}$, D. Fassouliotis ${ }^{9}$, A. Favareto ${ }^{50 \mathrm{a}, 50 \mathrm{~b}}$, L. Fayard ${ }^{116}$, P. Federic ${ }^{145 a}$, O. L. Fedin ${ }^{122, \text { j, W. Fedorko }}{ }^{169}$, M. Fehling-Kaschek ${ }^{48}$, S. Feigl ${ }^{30}$, L. Feligioni ${ }^{84}$, C. Feng ${ }^{33 d}$, E. J. Feng ${ }^{6}$, H. Feng ${ }^{88}$, A. B. Fenyuk ${ }^{129}$, S. Fernandez Perez ${ }^{30}$, S. Ferrag ${ }^{53}$, J. Ferrando ${ }^{53}$, A. Ferrari ${ }^{167}$, P. Ferrari ${ }^{106}$, R. Ferrari ${ }^{120 a}$, D. E. Ferreira de Lima ${ }^{53}$, A. Ferrer ${ }^{168}$, D. Ferrere ${ }^{49}$, C. Ferretti ${ }^{88}$, A. Ferretto Parodi ${ }^{50 a, 50 b}$, M. Fiascaris ${ }^{31}$, F. Fiedler ${ }^{82}$, A. Filipčič ${ }^{74}$, M. Filipuzzi ${ }^{42}$, F. Filthaut ${ }^{105}$, M. Fincke-Keeler ${ }^{170}$, K. D. Finelli1 ${ }^{151}$, M. C. N. Fiolhais ${ }^{125 a, 125 c}$, L. Fiorini ${ }^{168}$, A. Firan ${ }^{40}$, A. Fischer ${ }^{2}$, J. Fischer ${ }^{176}$, W. C. Fisher ${ }^{89}$, E. A. Fitzgerald ${ }^{23}$, M. Flechl ${ }^{48}$, I. Fleck ${ }^{142}$, P. Fleischmann ${ }^{88}$, S. Fleischmann ${ }^{176}$, G. T. Fletcher ${ }^{140}$, G. Fletcher $^{75}$, T. Flick ${ }^{176}$, A. Floderus ${ }^{80}$, L. R. Flores Castillo ${ }^{174 k}$, A. C. Florez Bustos ${ }^{160 b}$, M. J. Flowerdew ${ }^{100}$, A. Formica ${ }^{137}$, A. Forti ${ }^{83}$, D. Fortin ${ }^{160 a}$, D. Fournier ${ }^{116}$, H. Fox ${ }^{71}$, S. Fracchia ${ }^{12}$, P. Francavilla ${ }^{79}$, M. Franchini20a,20b,

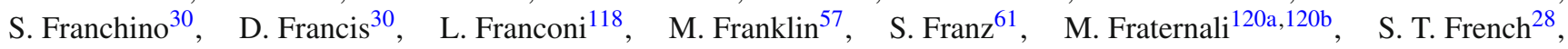
C. Friedrich ${ }^{42}$, F. Friedrich ${ }^{44}$, D. Froidevaux ${ }^{30}$, J. A. Frost ${ }^{28}$, C. Fukunaga ${ }^{157}$, E. Fullana Torregrosa ${ }^{82}$, B. G. Fulsom ${ }^{144}$, J. Fuster ${ }^{168}$, C. Gabaldon ${ }^{55}$, O. Gabizon ${ }^{173}$, A. Gabrielli20a,20b, A. Gabrielli ${ }^{133 a, 133 b}$, S. Gadatsch ${ }^{106}$, S. Gadomski ${ }^{49}$, G. Gagliardi50a,50b, P. Gagnon ${ }^{60}$, C. Galea ${ }^{105}$, B. Galhardo ${ }^{125 a, 125 c}$, E. J. Gallas ${ }^{119}$, V. Gallo ${ }^{17}$, B. J. Gallop ${ }^{130}$, P. Gallus ${ }^{127}$, G. Galster ${ }^{36}$, K. K. Gan ${ }^{110}$, J. Gao ${ }^{33 b, g}$, Y. S. Gao ${ }^{144, e}$, F. M. Garay Walls ${ }^{46}$, F. Garberson ${ }^{177}$, C. García ${ }^{168}$, J. E. García Navarro ${ }^{168}$, M. Garcia-Sciveres ${ }^{15}$, R. W. Gardner ${ }^{31}$, N. Garelli1 ${ }^{144}$, V. Garonne ${ }^{30}$, C. Gatti ${ }^{47}$, G. Gaudio ${ }^{120 a}$, B. Gaur ${ }^{142}$, L. Gauthier ${ }^{94}$, P. Gauzzi ${ }^{133 a, 133 b}$, I. L. Gavrilenko ${ }^{95}$, C. Gay ${ }^{169}$, G. Gaycken ${ }^{21}$, E. N. Gazis ${ }^{10}$, P. Ge ${ }^{33 d}$, Z. Gecse ${ }^{169}$, C. N. P. Gee ${ }^{130}$, D. A. A. Geerts ${ }^{106}$, Ch. Geich-Gimbel ${ }^{21}$, K. Gellerstedt ${ }^{147 a, 147 b}$, C. Gemme ${ }^{50 a}$, A. Gemmell ${ }^{53}$, M. H. Genest ${ }^{55}$, S. Gentile ${ }^{133 a, 133 b}$, M. George ${ }^{54}$, S. George ${ }^{76}$, D. Gerbaudo ${ }^{164}$, A. Gershon ${ }^{154}$, H. Ghazlane ${ }^{136 b}$, N. Ghodbane ${ }^{34}$, B. Giacobbe ${ }^{20 a}$, S. Giagu ${ }^{133 a, 133 b}$, V. Giangiobbe ${ }^{12}$, P. Giannetti 123a,123b, F. Gianotti ${ }^{30}$, B. Gibbard ${ }^{25}$, S. M. Gibson ${ }^{76}$, M. Gilchriese ${ }^{15}$, T. P. S. Gillam ${ }^{28}$, D. Gillberg ${ }^{30}$ ， G. Gilles ${ }^{34}$, D. M. Gingrich ${ }^{3, d}$, N. Giokaris ${ }^{9}$, M. P. Giordani ${ }^{165 a, 165 c}$, R. Giordano ${ }^{103 a, 103 b}$, F. M. Giorgi ${ }^{20 a}$, F. M. Giorgi ${ }^{16}$, P. F. Giraud ${ }^{137}$, D. Giugni ${ }^{90 a}$, C. Giuliani ${ }^{48}$,

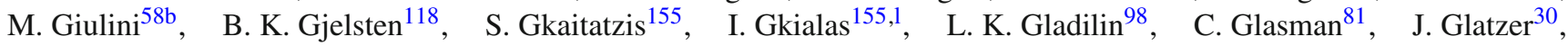
P. C. F. Glaysher ${ }^{46}$, A. Glazov ${ }^{42}$, G. L. Glonti ${ }^{64}$, M. Goblirsch-Kolb ${ }^{100}$, J. R. Goddard ${ }^{75}$, J. Godfrey ${ }^{143}$, J. Godlewski ${ }^{30}$, C. Goeringer ${ }^{82}$, S. Goldfarb ${ }^{88}$, T. Golling ${ }^{177}$, D. Golubkov ${ }^{129}$, A. Gomes ${ }^{125 a, 125 b, 125 d}$, L. S. Gomez Fajardo ${ }^{42}$, R. Gonçalo ${ }^{125 a}$, J. Goncalves Pinto Firmino Da Costa ${ }^{137}$, L. Gonella ${ }^{21}$, S. González de la Hoz ${ }^{168}$, G. Gonzalez Parra ${ }^{12}$, S. Gonzalez-Sevilla ${ }^{49}$, L. Goossens ${ }^{30}$, P. A. Gorbounov ${ }^{96}$, H. A. Gordon ${ }^{25}$, I. Gorelov ${ }^{104}$, B. Gorini ${ }^{30}$, E. Gorini ${ }^{72 a, 72 b}$, A. Gorišek ${ }^{74}$ ， E. Gornicki ${ }^{39}$ ， A. T. Goshaw ${ }^{6}$, C. Gössling ${ }^{43}$ ， M. I. Gostkin ${ }^{64}$, M. Gouighri136a， D. Goujdami ${ }^{136 c}$, M. P. Goulette ${ }^{49}$, A. G. Goussiou ${ }^{139}$, C. Goy ${ }^{5}$, S. Gozpinarr ${ }^{23}$, H. M. X. Grabas ${ }^{137}$, L. Graber ${ }^{54}$, I. Grabowska-Bold ${ }^{38 a}$, 
P. Grafström ${ }^{20 a, 20 b}$ ， K.-J. Grahn ${ }^{42}$ ， J. Gramling ${ }^{49}$ ， E. Gramstad ${ }^{118}$ ， S. Grancagnolo ${ }^{16}$ ， V. Grassi ${ }^{149}$ ， V. Gratchev ${ }^{122}$, H. M. Gray ${ }^{30}$, E. Graziani ${ }^{135 a}$, O. G. Grebenyuk ${ }^{122}$, Z. D. Greenwood ${ }^{78, m}$, K. Gregersen ${ }^{77}$, I. M. Gregor ${ }^{42}$, P. Grenier ${ }^{144}$, J. Griffiths ${ }^{8}$, A. A. Grillo ${ }^{138}$, K. Grimm ${ }^{71}$, S. Grinstein ${ }^{12, n}$, Ph. Gris ${ }^{34}$, Y. V. Grishkevich ${ }^{98}$, J.-F. Grivaz ${ }^{116}$, J. P. Grohs ${ }^{44}$, A. Grohsjean ${ }^{42}$, E. Gross ${ }^{173}$, J. Grosse-Knetter ${ }^{54}$, G. C. Grossi ${ }^{134 a, 134 b}$, J. Groth-Jensen ${ }^{173}$, Z. J. Grout ${ }^{150}$, L. Guan ${ }^{33 b}$, F. Guescini ${ }^{49}$, D. Guest ${ }^{177}$, O. Gueta ${ }^{154}$, C. Guicheney ${ }^{34}$, E. Guido ${ }^{50 a, 50 b}$, T. Guillemin ${ }^{116}$, S. Guindon ${ }^{2}$, U. Gul ${ }^{53}$, C. Gumpert ${ }^{44}$, J. Gunther ${ }^{127}$, J. Guo ${ }^{35}$, S. Gupta ${ }^{119}$, P. Gutierrez ${ }^{112}$, N. G. Gutierrez Ortiz ${ }^{53}$, C. Gutschow ${ }^{77}$, N. Guttman ${ }^{154}$, C. Guyot ${ }^{137}$, C. Gwenlan ${ }^{119}$, C. B. Gwilliam ${ }^{73}$, A. Haas ${ }^{109}$, C. Haber ${ }^{15}$, H. K. Hadavand ${ }^{8}$, N. Haddad ${ }^{136 e}$, P. Haefner ${ }^{21}$, S. Hageböeck ${ }^{21}$, Z. Hajduk ${ }^{39}$, H. Hakobyan ${ }^{178}$, M. Haleem ${ }^{42}$, D. Hall ${ }^{119}$, G. Halladjian ${ }^{89}$, K. Hamacher ${ }^{176}$, P. Hamal ${ }^{114}$, K. Hamano ${ }^{170}$, M. Hamer ${ }^{54}$, A. Hamilton ${ }^{146 a}$, S. Hamilton ${ }^{162}$, G. N. Hamity ${ }^{146 c}$, P. G. Hamnett ${ }^{42}$, L. Han ${ }^{33 b}$, K. Hanagaki ${ }^{117}$, K. Hanawa ${ }^{156}$, M. Hance ${ }^{15}$, P. Hanke ${ }^{58 a}$, R. Hann ${ }^{137}$, J. B. Hansen ${ }^{36}$ ，J. D. Hansen ${ }^{36}$, P. H. Hansen ${ }^{36}$,

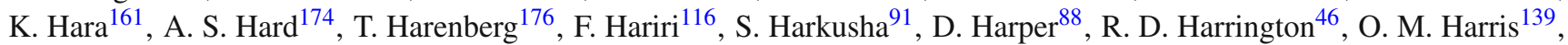
P. F. Harrison ${ }^{171}$, F. Hartjes ${ }^{106}$, M. Hasegawa ${ }^{66}$, S. Hasegawa ${ }^{102}$, Y. Hasegawa ${ }^{141}$, A. Hasib ${ }^{112}$, S. Hassani ${ }^{137}$, S. Haug ${ }^{17}$, M. Hauschild ${ }^{30}$, R. Hauser ${ }^{89}$, M. Havranek ${ }^{126}$, C. M. Hawkes ${ }^{18}$, R. J. Hawkings ${ }^{30}$, A. D. Hawkins ${ }^{80}$, T. Hayashi ${ }^{161}$,

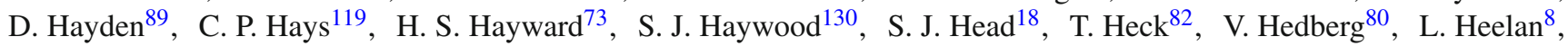
S. Heim ${ }^{121}$, T. Heim ${ }^{176}$, B. Heinemann ${ }^{15}$, L. Heinrich ${ }^{109}$, J. Hejbal ${ }^{126}$, L. Helary ${ }^{22}$, C. Heller99, M. Heller ${ }^{30}$, S. Hellman ${ }^{147 a, 147 b}$, D. Hellmich ${ }^{21}$, C. Helsens ${ }^{30}$, J. Henderson ${ }^{119}$, R. C. W. Henderson ${ }^{71}$, Y. Heng ${ }^{174}$, C. Hengler ${ }^{42}$, A. Henrichs ${ }^{177}$, A. M. Henriques Correia ${ }^{30}$, S. Henrot-Versille ${ }^{116}$, C. Hensel ${ }^{54}$, G. H. Herbert ${ }^{16}$, Y. Hernández Jiménez ${ }^{168}$, R. Herrberg-Schubert ${ }^{16}$, G. Herten ${ }^{48}$, R. Hertenberger ${ }^{99}$, L. Hervas ${ }^{30}$, G. G. Hesketh ${ }^{77}$, N. P. Hessey ${ }^{106}$, R. Hickling ${ }^{75}$, E. Higón-Rodriguez ${ }^{168}$, E. Hill ${ }^{170}$, J. C. Hill ${ }^{28}$, K. H. Hiller ${ }^{42}$, S. Hillert ${ }^{21}$, S. J. Hillier ${ }^{18}$, I. Hinchliffe ${ }^{15}$, E. Hines ${ }^{121}$,

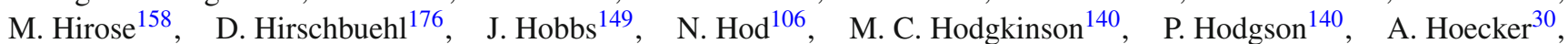
M. R. Hoeferkamp ${ }^{104}$, F. Hoenig 99 , J. Hoffman ${ }^{40}$, D. Hoffmann ${ }^{84}$, J. I. Hofmann58a, M. Hohlfeld ${ }^{82}$, T. R. Holmes ${ }^{15}$, T. M. Hong ${ }^{121}$, L. Hooft van Huysduynen ${ }^{109}$, Y. Horii ${ }^{102}$, J.-Y. Hostachy ${ }^{55}$, S. Hou ${ }^{152}$, A. Hoummada ${ }^{136 a}$, J. Howard ${ }^{119}$, J. Howarth ${ }^{42}$, M. Hrabovsky ${ }^{114}$, I. Hristova ${ }^{16}$, J. Hrivnac ${ }^{116}$, T. Hryn'ova ${ }^{5}$, C. Hsu ${ }^{146 c}$, P. J. Hsu ${ }^{82}$, S.-C. Hsu ${ }^{139}$, D. Hu ${ }^{35}$, X. $\mathrm{Hu}^{25}$, Y. Huang ${ }^{42}$, Z. Hubacek ${ }^{30}$, F. Hubaut ${ }^{84}$, F. Huegging ${ }^{21}$, T. B. Huffman ${ }^{119}$, E. W. Hughes ${ }^{35}$, G. Hughes ${ }^{71}$,

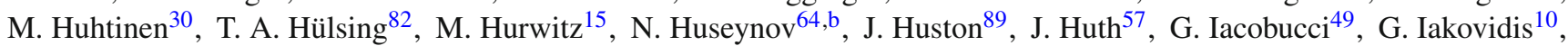
I. Ibragimov $^{142}$, L. Iconomidou-Fayard ${ }^{116}$, E. Ideal ${ }^{177}, \quad$ P. Iengo ${ }^{103 a}$ ， O. Igonkina ${ }^{106}, \quad$ T. Iizawa ${ }^{172}$, Y. Ikegami ${ }^{65}$, K. Ikematsu ${ }^{142}$, M. Ikeno $^{65}$, Y. Ilchenko ${ }^{31,0}$, D. Iliadis ${ }^{155}$, N. Ilic ${ }^{159}$, Y. Inamaru ${ }^{66}$, T. Ince ${ }^{100}$, P. Ioannou ${ }^{9}$, M. Iodice $^{135 a}$, K. Iordanidou ${ }^{9}$, V. Ippolito ${ }^{57}$, A. Irles Quiles ${ }^{168}$, C. Isaksson ${ }^{167}$, M. Ishino ${ }^{67}$, M. Ishitsuka ${ }^{158}$, R. Ishmukhametov ${ }^{110}$, C. Issever ${ }^{119}$, S. Istin ${ }^{19 a}$, J. M. Iturbe Ponce ${ }^{83}$, R. Iuppa ${ }^{134 a, 134 b}$, J. Ivarsson ${ }^{80}$, W. Iwanski ${ }^{39}$, H. Iwasaki ${ }^{65}$, J. M. Izen ${ }^{41}$, V. Izzo ${ }^{103 a}$, B. Jackson ${ }^{121}, \quad$ M. Jackson ${ }^{73}$, P. Jackson ${ }^{1}, \quad$ M. R. Jaekel ${ }^{30}, \quad$ V. Jain ${ }^{2}, \quad$ K. Jakobs ${ }^{48}, \quad$ S. Jakobsen ${ }^{30}$, T. Jakoubek ${ }^{126}$ ，J. Jakubek ${ }^{127}$ ， D. O. Jamin ${ }^{152}$ ， D. K. Jana ${ }^{78}$ ， E. Jansen ${ }^{77}$ ， H. Jansen ${ }^{30 ， \quad J . ~ J a n s s e n ~}{ }^{21}, \quad$ M. Janus ${ }^{171}$, G. Jarlskog ${ }^{80}$, N. Javadov ${ }^{64, b}$, T. Javůrek ${ }^{48}$, L. Jeanty ${ }^{15}$, J. Jejelava ${ }^{51 a, p}$ ， G.-Y. Jeng ${ }^{151}$ ， D. Jennens ${ }^{87}$, P. Jenni ${ }^{48, q}$, J. Jentzsch ${ }^{43}$, C. Jeske ${ }^{171}$, S. Jézéquel ${ }^{5}$, H. Ji ${ }^{174}$, J. Jia ${ }^{149}$, Y. Jiang ${ }^{33 b}$, M. Jimenez Belenguer ${ }^{42}$, S. Jin ${ }^{33 a}$, A. Jinaru ${ }^{26 a}$, O. Jinnouchi ${ }^{158}$, M. D. Joergensen ${ }^{36}$, K. E. Johansson ${ }^{147 a, 147 b}$, P. Johansson ${ }^{140}$, K. A. Johns ${ }^{7}$, K. Jon-And ${ }^{147 a, 147 b}$, G. Jones ${ }^{171}$, R. W. L. Jones ${ }^{71}$, T. J. Jones ${ }^{73}$, J. Jongmanns ${ }^{58 a}$, P. M. Jorge ${ }^{125 a, 125 b}$, K. D. Joshi ${ }^{83}$, J. Jovicevic ${ }^{148}$, X. Ju ${ }^{174}$, C. A. Jung ${ }^{43}$, R. M. Jungst ${ }^{30}$, P. Jussel ${ }^{61}$, A. Juste Rozas ${ }^{12, n}$, M. Kaci ${ }^{168}$, A. Kaczmarska ${ }^{39}$, M. Kado ${ }^{116}$, H. Kagan ${ }^{110}$, M. Kagan ${ }^{144}$, E. Kajomovitz ${ }^{45}$, C. W. Kalderon ${ }^{119}$, S. Kama ${ }^{40}$, A. Kamenshchikov ${ }^{129}$, N. Kanaya ${ }^{156}$, M. Kaneda ${ }^{30}$, S. Kaneti ${ }^{28}$, V. A. Kantserov ${ }^{97}$, J. Kanzaki ${ }^{65}$, B. Kaplan ${ }^{109}$, A. Kapliy ${ }^{31}$, D. Kar ${ }^{53}$, K. Karakostas ${ }^{10}$, N. Karastathis ${ }^{10}$, M. Karnevskiy ${ }^{82}$, S. N. Karpov ${ }^{64}$, Z. M. Karpova ${ }^{64}$, K. Karthik ${ }^{109}$, V. Kartvelishvili ${ }^{71}$, A. N. Karyukhin ${ }^{129}$, L. Kashif ${ }^{174}$, G. Kasieczka ${ }^{58 b}$, R. D. Kass ${ }^{110}$, A. Kastanas ${ }^{14}$, Y. Kataoka ${ }^{156}$, A. Katre ${ }^{49}$ ， J. Katzy ${ }^{42}$, V. Kaushik ${ }^{7}$, K. Kawagoe ${ }^{69}$, T. Kawamoto ${ }^{156}$, G. Kawamura ${ }^{54}$, S. Kazama ${ }^{156}$, V. F. Kazanin ${ }^{108}$, M. Y. Kazarinov ${ }^{64}$, R. Keeler ${ }^{170}$, R. Kehoe ${ }^{40}$, M. Keil ${ }^{54}$, J. S. Keller ${ }^{42}$, J. J. Kempster ${ }^{76}$, H. Keoshkeriann, O. Kepka ${ }^{126}$, B. P. Kerševan ${ }^{74}$, S. Kersten ${ }^{176}$, K. Kessoku ${ }^{156}$, J. Keung ${ }^{159}$, F. Khalil-zada ${ }^{11}$, H. Khandanyan ${ }^{147 a, 147 b}$, A. Khanov ${ }^{113}$, A. Khodinov ${ }^{97}$, A. Khomich ${ }^{58 a}$, T. J. Khoo ${ }^{28}$, G. Khoriauli ${ }^{21}$, A. Khoroshilov ${ }^{176}$, V. Khovanskiy ${ }^{96}$, E. Khramov ${ }^{64}$, J. Khubua ${ }^{51 b}$, H. Y. Kim ${ }^{8}, \quad$ H. Kim ${ }^{147 a, 147 b}$,

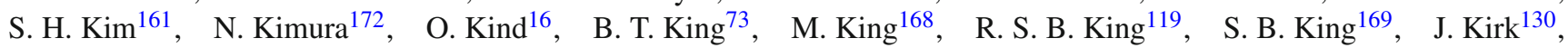
A. E. Kiryunin ${ }^{100}$, T. Kishimoto ${ }^{66}$, D. Kisielewska ${ }^{38 a}$, F. Kiss ${ }^{48}$, T. Kittelmann ${ }^{124}$, K. Kiuchi ${ }^{161}, \quad$ E. Kladiva ${ }^{145 b}$, M. Klein ${ }^{73}$, U. Klein ${ }^{73}$, K. Kleinknecht ${ }^{82}$, P. Klimek ${ }^{147 a, 147 b}$, A. Klimentov ${ }^{25}$, R. Klingenberg ${ }^{43}$, J. A. Klinger ${ }^{83}$, T. Klioutchnikova ${ }^{30}$, P. F. Klok ${ }^{105}$ ， E.-E. Kluge ${ }^{58 a}$, P. Kluit ${ }^{106}$, S. Kluth ${ }^{100}$, E. Kneringer ${ }^{61}$, E. B. F. G. Knoops ${ }^{84}$, A. Knue ${ }^{53}$, D. Kobayashi ${ }^{158}$, T. Kobayashi ${ }^{156}$, M. Kobel ${ }^{44}$, M. Kocian ${ }^{144}$, P. Kodys ${ }^{128}$, P. Koevesarki ${ }^{21}$, T. Koffas ${ }^{29}$, E. Koffeman ${ }^{106}$, L. A. Kogan ${ }^{119}$, S. Kohlmann ${ }^{176}$, Z. Kohout ${ }^{127}$, T. Kohriki ${ }^{65}$, T. Koi ${ }^{144}$, H. Kolanoski ${ }^{16}$, I. Koletsou ${ }^{5}$, J. Koll ${ }^{89}$, A. A. Komar ${ }^{95, *}$, Y. Komori ${ }^{156}$, T. Kondo ${ }^{65}$, N. Kondrashova ${ }^{42}$, K. Köneke ${ }^{48}$, A. C. König ${ }^{105}$, S. König ${ }^{82}$, T. Kono ${ }^{65, \mathrm{r}}$, R. Konoplich ${ }^{109, \mathrm{~s}}$, N. Konstantinidis ${ }^{77}$, R. Kopeliansky ${ }^{153}$ ， S. Koperny ${ }^{38 a}$ ， L. Köpke ${ }^{82}$, A. K. Kopp ${ }^{48}$, K. Korcyl $^{39}$, K. Kordas ${ }^{155}$, A. Korn ${ }^{77}$, A. A. Korol ${ }^{108, t}$, I. Korolkov ${ }^{12}$, E. V. Korolkova ${ }^{140}$, V. A. Korotkov ${ }^{129}$, 
O. Kortner ${ }^{100}$ ， S. Kortner ${ }^{100}$ ， V. V. Kostyukhin ${ }^{21}$ ， V. M. Kotov ${ }^{64}$ ，A. Kotwal ${ }^{45}$ ， C. Kourkoumelis ${ }^{9}$ ， V. Kouskoura ${ }^{155}$, A. Koutsman ${ }^{160 a}$, R. Kowalewski ${ }^{170}$, T. Z. Kowalski ${ }^{38 a}$, W. Kozanecki ${ }^{137}$, A. S. Kozhin ${ }^{129}$, V. Kral ${ }^{127}$, V. A. Kramarenko ${ }^{98}$, G. Kramberger ${ }^{74}$, D. Krasnopevtsev ${ }^{97}$, M. W. Krasny ${ }^{79}$, A. Krasznahorkay ${ }^{30}$, J. K. Kraus ${ }^{21}$, A. Kravchenko ${ }^{25}$, S. Kreiss ${ }^{109}$, M. Kretz ${ }^{58 c}$, J. Kretzschmar ${ }^{73}$, K. Kreutzfeldt ${ }^{52}$, P. Krieger ${ }^{159}$, K. Kroeninger ${ }^{54}$, H. Kroha ${ }^{100}$, J. Kroll ${ }^{121}$, J. Kroseberg ${ }^{21}$, J. Krstic ${ }^{13 a}$, U. Kruchonak ${ }^{64}$, H. Krüger ${ }^{21}$, T. Kruker $^{17}$, N. Krumnack ${ }^{63}$, Z. V. Krumshteyn ${ }^{64}$, A. Kruse ${ }^{174}$, M. C. Kruse ${ }^{45}$, M. Kruskal ${ }^{22}$, T. Kubota ${ }^{87}$, S. Kuday ${ }^{4 a}$, S. Kuehn ${ }^{48}$, A. Kugel $^{58 c}$, A. Kuhl ${ }^{138}$, T. Kuhl ${ }^{42}$, V. Kukhtin ${ }^{64}$, Y. Kulchitsky ${ }^{91}$, S. Kuleshov ${ }^{32 b}$, M. Kuna ${ }^{133 a, 133 b}$, J. Kunkle ${ }^{121}$, A. Kupco ${ }^{126}$, H. Kurashige ${ }^{66}$, Y. A. Kurochkin ${ }^{91}$, R. Kurumida ${ }^{66}$, V. Kus ${ }^{126}$, E. S. Kuwertz ${ }^{148}$, M. Kuze ${ }^{158}$, J. Kvita ${ }^{114}$, A. La Rosa ${ }^{49}$, L. La Rotonda ${ }^{37 a, 37 b}$, C. Lacasta ${ }^{168}$, F. Lacava ${ }^{133 a, 133 b}$,

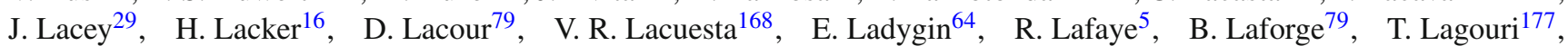
S. Lai $^{48}$, H. Laier ${ }^{58 a}$, L. Lambourne ${ }^{77}$, S. Lammers ${ }^{60}$, C. L. Lampen ${ }^{7}$, W. Lampl ${ }^{7}$, E. Lançon ${ }^{137}$, U. Landgraf ${ }^{48}$,

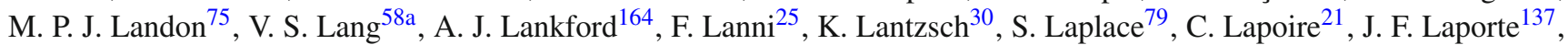
T. Lari ${ }^{90 a}$, M. Lassnig ${ }^{30}$, P. Laurelli ${ }^{47}$, W. Lavrijsen ${ }^{15}$, A. T. Law ${ }^{138}$, P. Laycock ${ }^{73}$, O. Le Dortz ${ }^{79}$, E. Le Guirriec ${ }^{84}$, E. Le Menedeu ${ }^{12}$, T. LeCompte ${ }^{6}$, F. Ledroit-Guillon ${ }^{55}$, C. A. Lee ${ }^{152}$, H. Lee ${ }^{106}$, J. S. H. Lee ${ }^{117}$, S. C. Lee ${ }^{152}$, L. Lee ${ }^{1}$, G. Lefebvre ${ }^{79}$, M. Lefebvre ${ }^{170}$, F. Legger ${ }^{99}$, C. Leggett ${ }^{15}$, A. Lehan ${ }^{73}$, M. Lehmacher ${ }^{21}$, G. Lehmann Miotto ${ }^{30}$, X. Lei ${ }^{7}$, W. A. Leight ${ }^{29}$, A. Leisos ${ }^{155}$, A. G. Leister ${ }^{177}$, M. A. L. Leite ${ }^{24 d}$, R. Leitner ${ }^{128}$, D. Lellouch ${ }^{173}$, B. Lemmer ${ }^{54}$, K. J. C. Leney ${ }^{77}$, T. Lenz ${ }^{21}$, G. Lenzen ${ }^{176}$, B. Lenzi ${ }^{30}$, R. Leone ${ }^{7}$, S. Leone ${ }^{123 a, 123 b}$, K. Leonhardt ${ }^{44}$, C. Leonidopoulos ${ }^{46}$, S. Leontsinis ${ }^{10}$, C. Leroy ${ }^{94}$, C. G. Lester ${ }^{28}$, C. M. Lester ${ }^{121}$, M. Levchenko ${ }^{122}$, J. Levêque ${ }^{5}$, D. Levin ${ }^{88}$, L. J. Levinson ${ }^{173}$, M. Levy ${ }^{18}$, A. Lewis ${ }^{119}$, G. H. Lewis ${ }^{109}$, A. M. Leyko ${ }^{21}$, M. Leyton ${ }^{41}$, B. Li ${ }^{33 b, u}, \quad$ B. Li ${ }^{84}, \quad$ H. Li ${ }^{149}, \quad$ H. L. Li ${ }^{31}$, L. $\mathrm{Li}^{45}$, L. Li ${ }^{33 \mathrm{e}}$, S. Li ${ }^{45}$, Y. Li ${ }^{33 \mathrm{c}, \mathrm{v}}$, Z. Liang ${ }^{138}, \quad$ H. Liao ${ }^{34}$, B. Liberti ${ }^{134 a}$, P. Lichard ${ }^{30}$, K. Lie ${ }^{166}$, J. Liebal ${ }^{21}$, W. Liebig ${ }^{14}$, C. Limbach ${ }^{21}$, A. Limosani ${ }^{87}$, S. C. $\operatorname{Lin}^{152, w}$, T. H. $\operatorname{Lin}^{82}$, F. Linde ${ }^{106}$, B. E. Lindquist ${ }^{149}$, J. T. Linnemann ${ }^{89}$, E. Lipeles ${ }^{121}$, A. Lipniacka ${ }^{14}$, M. Lisovyi ${ }^{42}$, T. M. Liss ${ }^{166}$, D. Lissauer ${ }^{25}$, A. Lister ${ }^{169}$, A. M. Litke ${ }^{138}$, B. Liu ${ }^{152}$, D. Liu ${ }^{152}$,

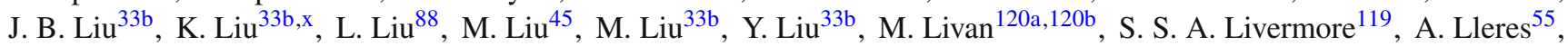
J. Llorente Merino ${ }^{81}$, S. L. Lloyd ${ }^{75}$, F. Lo Sterzo ${ }^{152}$, E. Lobodzinska ${ }^{42}$, P. Loch ${ }^{7}$, W. S. Lockman ${ }^{138}$, T. Loddenkoetter ${ }^{21}$, F. K. Loebinger ${ }^{83}$, A. E. Loevschall-Jensen ${ }^{36}, \quad$ A. Loginov ${ }^{177}, \quad$ T. Lohse ${ }^{16}, \quad$ K. Lohwasser ${ }^{42}, \quad$ M. Lokajicek ${ }^{126}$, V. P. Lombardo ${ }^{5}$, B. A. Long ${ }^{22}$, J. D. Long ${ }^{88}$, R. E. Long ${ }^{71}$, L. Lopes ${ }^{125 a}$, D. Lopez Mateos ${ }^{57}$, B. Lopez Paredes ${ }^{140}$, I. Lopez Paz ${ }^{12}$, J. Lorenz ${ }^{99}$, N. Lorenzo Martinez ${ }^{60}$, M. Losada ${ }^{163}$, P. Loscutoff ${ }^{15}$, X. Lou ${ }^{41}$, A. Lounis ${ }^{116}$, J. Love ${ }^{6}$, P. A. Love ${ }^{71}$, A. J. Lowe ${ }^{144, e}$, F. Lu ${ }^{33 a}$, N. Lu ${ }^{88}$, H. J. Lubatti ${ }^{139}$, C. Luci ${ }^{133 a, 133 b}$, A. Lucotte ${ }^{55}$, F. Luehring ${ }^{60}$, W. Lukas ${ }^{61}$, L. Luminari ${ }^{133 a}$, O. Lundberg ${ }^{147 a, 147 b}, \quad$ B. Lund-Jensen ${ }^{148}, \quad$ M. Lungwitz ${ }^{82}, \quad$ D. Lynn ${ }^{25}, \quad$ R. Lysak ${ }^{126}$, E. Lytken ${ }^{80}$, H. Ma ${ }^{25}$, L. L. Ma ${ }^{33 d}$, G. Maccarrone ${ }^{47}$, A. Macchiolo ${ }^{100}$, J. Machado Miguens ${ }^{125 a, 125 b}$, D. Macina ${ }^{30}$, D. Madaffari ${ }^{84}$, R. Madar ${ }^{48}$, H. J. Maddocks ${ }^{71}$, W. F. Mader ${ }^{44}$, A. Madsen ${ }^{167}$, M. Maeno ${ }^{8}$, T. Maeno ${ }^{25}$, E. Magradze ${ }^{54}$, K. Mahboubi ${ }^{48}$, J. Mahlstedt ${ }^{106}$, S. Mahmoud ${ }^{73}$, C. Maiani ${ }^{137}$, C. Maidantchik ${ }^{24 a}$, A. A. Maier ${ }^{100}$, A. Maio ${ }^{125 a, 125 b, 125 d,}$ S. Majewski ${ }^{115}$, Y. Makida ${ }^{65}$, N. Makovec ${ }^{116}$, P. Mal ${ }^{137, y}$, B. Malaescu ${ }^{79}$, Pa. Malecki ${ }^{39}$, V. P. Maleev ${ }^{122}$, F. Malek ${ }^{55}$, U. Mallik ${ }^{62}$, D. Malon ${ }^{6}$, C. Malone ${ }^{144}$, S. Maltezos ${ }^{10}$, V. M. Malyshev ${ }^{108}$, S. Malyukov ${ }^{30}$, J. Mamuzic ${ }^{13 b}$, B. Mandelli ${ }^{30}$, L. Mandelli ${ }^{90 a}$, I. Mandić ${ }^{74}$, R. Mandrysch ${ }^{62}$, J. Maneira ${ }^{125 a, 125 b}$, A. Manfredini ${ }^{100}$, L. Manhaes de Andrade Filho ${ }^{24 b}$, J. A. Manjarres Ramos ${ }^{160 b}$, A. Mann ${ }^{99}$, P. M. Manning ${ }^{138}$, A. Manousakis-Katsikakis ${ }^{9}$, B. Mansoulie ${ }^{137}$, R. Mantifel ${ }^{86}$, L. Mapelli ${ }^{30}$, L. March ${ }^{168}$, J. F. Marchand ${ }^{29}$, G. Marchiori ${ }^{79}$, M. Marcisovsky ${ }^{126}$, C. P. Marino ${ }^{170}$, M. Marjanovic ${ }^{13 a}$, C. N. Marques ${ }^{125 a}$, F. Marroquim ${ }^{24 a}$, S. P. Marsden ${ }^{83}$, Z. Marshall ${ }^{15}$, L. F. Marti ${ }^{17}$, S. Marti-Garcia ${ }^{168}$, B. Martin ${ }^{30}$, B. Martin ${ }^{89}$, T. A. Martin ${ }^{171}$, V. J. Martin ${ }^{46}$, B. Martin dit Latour ${ }^{14}$, H. Martinez ${ }^{137}$, M. Martinez ${ }^{12, n}$, S. Martin-Haugh ${ }^{130}$, A. C. Martyniuk ${ }^{77}$, M. Marx ${ }^{139}$, F. Marzano ${ }^{133 a}$, A. Marzin $^{30}$, L. Masetti ${ }^{82}$, T. Mashimo ${ }^{156}$, R. Mashinistov ${ }^{95}$, J. Masik ${ }^{83}$, A. L. Maslennikov ${ }^{108}$, I. Massa ${ }^{20 a, 20 b}$, L. Massa ${ }^{20 a, 20 b}$, N. Massol ${ }^{5}$, P. Mastrandrea ${ }^{149}$, A. Mastroberardino ${ }^{37 a, 37 b}$, T. Masubuchi ${ }^{156}$, P. Mättig ${ }^{176}$, J. Mattmann ${ }^{82}$, J. Maurer ${ }^{26 a}$, S. J. Maxfield ${ }^{73}$, D. A. Maximov 108,t, R. Mazini ${ }^{152}$, L. Mazzaferro ${ }^{134 a, 134 b}$, G. Mc Goldrick ${ }^{159}$, S. P. Mc Kee ${ }^{88}$, A. McCarn ${ }^{88}$, R. L. McCarthy ${ }^{149}$, T. G. McCarthy ${ }^{29}$, N. A. McCubbin ${ }^{130}$, K. W. McFarlane ${ }^{56, *}$, J. A. $\operatorname{Mcfayden}^{77}$, G. Mchedlidze ${ }^{54}$, S. J. McMahon ${ }^{130}$, R. A. McPherson ${ }^{170, i}$, A. Meade ${ }^{85}$, J. Mechnich ${ }^{106}$, M. Medinnis ${ }^{42}$, S. Meehan ${ }^{31}$, S. Mehlhase ${ }^{99}$, A. Mehta ${ }^{73}$, K. Meier ${ }^{58 a}$, C. Meineck ${ }^{99}$, B. Meirose ${ }^{80}$, C. Melachrinos ${ }^{31}$, B. R. Mellado Garcia ${ }^{146 c}$, F. Meloni17, A. Mengarelli20a,20b, S. Menke ${ }^{100}$, E. Meoni ${ }^{162}$, K. M. Mercurio ${ }^{57}$, S. Mergelmeyer ${ }^{21}$, N. Meric ${ }^{137}$, P. Mermod ${ }^{49}$, L. Merola ${ }^{103 a, 103 b}$, C. Meroni ${ }^{90 a}$, F. S. Merritt ${ }^{31}$, H. Merritt ${ }^{110}$, A. Messina ${ }^{30, z}$, J. Metcalfe ${ }^{25}$, A. S. Mete ${ }^{164}$, C. Meyer ${ }^{82}$, C. Meyer ${ }^{121}$, J.-P. Meyer ${ }^{137}$, J. Meyer ${ }^{30}$,

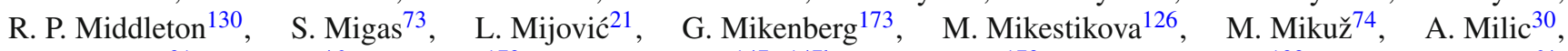
D. W. Miller ${ }^{31}$, C. Mills ${ }^{46}$, A. Milov ${ }^{173}$, D. A. Milstead ${ }^{147 a, 147 b}$, D. Milstein ${ }^{173}$, A. A. Minaenko ${ }^{129}$, I. A. Minashvili ${ }^{64}$, A. I. Mincer ${ }^{109}$, B. Mindur ${ }^{38 a}$, M. Mineev ${ }^{64}$, Y. Ming ${ }^{174}$, L. M. Mir ${ }^{12}$, G. Mirabelli ${ }^{133 a}$, T. Mitani ${ }^{172}$, J. Mitrevski ${ }^{99}$, V. A. Mitsou ${ }^{168}$, S. Mitsui ${ }^{65}$, A. Miucci ${ }^{49}$, P. S. Miyagawa ${ }^{140}$, J. U. Mjörnmark ${ }^{80}$, T. Moa ${ }^{147 a, 147 b}$, K. Mochizuki ${ }^{84}$, S. Mohapatra ${ }^{35}$, W. Mohr ${ }^{48}$, S. Molander ${ }^{147 a, 147 b}$, R. Moles-Valls ${ }^{168}, \quad$ K. Mönig ${ }^{42}, \quad$ C. Monini ${ }^{55}$, J. Monk ${ }^{36}$, E. Monnier $^{84}$, J. Montejo Berlingen ${ }^{12}$, F. Monticelli ${ }^{70}$, S. Monzani ${ }^{133 a, 133 b}$, R. W. Moore ${ }^{3}$, N. Morange ${ }^{62}$, D. Moreno ${ }^{82}$, 
M. Moreno Llácer ${ }^{54}$ ，P. Morettini50a，M. Morgenstern ${ }^{44}$ ，M. Morii ${ }^{57}$ ， S. Moritz ${ }^{82}$ ，A. K. Morley ${ }^{148}$ ， G. Mornacchi ${ }^{30}$, J. D. Morris ${ }^{75}$, L. Morvaj ${ }^{102}$, H. G. Moser ${ }^{100}$, M. Mosidze ${ }^{51 b}$, J. Moss ${ }^{110}$, K. Motohashi ${ }^{158}$, R. Mount ${ }^{144}$, E. Mountricha ${ }^{25}$, S. V. Mouraviev ${ }^{95, *}$, E. J. W. Moyse ${ }^{85}$, S. Muanza ${ }^{84}$, R. D. Mudd ${ }^{18}$, F. Mueller ${ }^{58 a}$, J. Mueller ${ }^{124}$, K. Mueller ${ }^{21}$, T. Mueller ${ }^{28}$, T. Mueller ${ }^{82}$, D. Muenstermann ${ }^{49}$, Y. Munwes ${ }^{154}$, J. A. Murillo Quijada ${ }^{18}$, W. J. Murray ${ }^{171,130}$, H. Musheghyan ${ }^{54}$, E. Musto ${ }^{153}$, A. G. Myagkov ${ }^{129, a a}$, M. Myska ${ }^{127}$, O. Nackenhorst ${ }^{54}$, J. Nadal ${ }^{54}$, K. Nagai ${ }^{61}$, R. Nagai ${ }^{158}$, Y. Nagai ${ }^{84}$, K. Nagano ${ }^{65}$, A. Nagarkar ${ }^{110}$, Y. Nagasaka ${ }^{59}$, M. Nagel ${ }^{100}$, A. M. Nairz ${ }^{30}$, Y. Nakahama ${ }^{30}$, K. Nakamura $^{65}$, T. Nakamura ${ }^{156}$, I. Nakano ${ }^{111}$, H. Namasivayam ${ }^{41}$, G. Nanava ${ }^{21}$, R. Narayan ${ }^{58 b}$, T. Nattermann ${ }^{21}$, T. Naumann ${ }^{42}$, G. Navarro ${ }^{163}$, R. Nayyar ${ }^{7}$, H. A. Neal ${ }^{88}$, P. Yu. Nechaeva ${ }^{95}$, T. J. Neep ${ }^{83}$, P. D. Nef ${ }^{144}$, A. Negri ${ }^{120 a, 120 b}$, G. Negri $^{30}$, M. Negrini ${ }^{20 a}$, S. Nektarijevic ${ }^{49}$, A. Nelson ${ }^{164}$, T. K. Nelson ${ }^{144}$, S. Nemecek ${ }^{126}$, P. Nemethy ${ }^{109}$, A. A. Nepomuceno ${ }^{24 a}$, M. Nessi ${ }^{30, a b}$, M. S. Neubauer ${ }^{166}$, M. Neumann ${ }^{176}$, R. M. Neves ${ }^{109}$, P. Nevski ${ }^{25}$, P. R. Newman ${ }^{18}$, D. H. Nguyen ${ }^{6}$, R. B. Nickerson ${ }^{119}$, R. Nicolaidou ${ }^{137}$, B. Nicquevert ${ }^{30}$, J. Nielsen ${ }^{138}$, N. Nikiforou ${ }^{35}$, A. Nikiforov ${ }^{16}$, V. Nikolaenko ${ }^{129}$,aa, I. Nikolic-Audit ${ }^{79}$, K. Nikolics ${ }^{49}$, K. Nikolopoulos ${ }^{18}$, P. Nilsson ${ }^{8}$, Y. Ninomiya ${ }^{156}$, A. Nisati ${ }^{133 a}$, R. Nisius ${ }^{100}$, T. Nobe ${ }^{158}$, L. Nodulman ${ }^{6}$, M. Nomachi ${ }^{117}$, I. Nomidis ${ }^{29}$, S. Norberg ${ }^{112}$, M. Nordberg ${ }^{30}$, O. Novgorodova ${ }^{44}$, S. Nowak ${ }^{100}$, M. Nozaki ${ }^{65}$, L. Nozka ${ }^{114}$, K. Ntekas ${ }^{10}$, G. Nunes Hanninger ${ }^{87}$, T. Nunnemann ${ }^{99}$, E. Nurse ${ }^{77}$, F. Nuti ${ }^{87}$, B. J. O’Brien ${ }^{46}$, F. O'grady ${ }^{7}$, D. C. O’Neil ${ }^{143}$, V. O’ Shea $^{53}$, F. G. Oakham ${ }^{29, d}$, H. Oberlack ${ }^{100}$, T. Obermann ${ }^{21}$, J. Ocariz ${ }^{79}$, A. Ochi ${ }^{66}$, M. I. Ochoa ${ }^{77}$ ， S. Oda ${ }^{69}$, S. Odaka ${ }^{65}$, H. Ogren ${ }^{60}$, A. Oh ${ }^{83}$, S. H. Oh ${ }^{45}$, C. C. Ohm ${ }^{15}$, H. Ohman ${ }^{167}$, W. Okamura ${ }^{117}$, H. Okawa ${ }^{25}$, Y. Okumura ${ }^{31}$, T. Okuyama ${ }^{156}$, A. Olariu ${ }^{26 a}$, A. G. Olchevski ${ }^{64}$, S. A. Olivares Pino ${ }^{46}$, D. Oliveira Damazio ${ }^{25}$, E. Oliver Garcia ${ }^{168}$, A. Olszewski ${ }^{39}$, J. Olszowska ${ }^{39}$, A. Onofre ${ }^{125 a, 125 e}$, P. U. E. Onyisi ${ }^{31, o}$, C. J. Oram ${ }^{160 a}$, M. J. Oreglia ${ }^{31}$, Y. Oren ${ }^{154}$, D. Orestano ${ }^{135 a, 135 b}$, N. Orlando ${ }^{72 a, 72 b}$, C. Oropeza Barrera ${ }^{53}$, R. S. Orr ${ }^{159}$, B. Osculati ${ }^{50 a, 50 b}$, R. Ospanov ${ }^{121}$, G. Otero y Garzon ${ }^{27}$, H. Otono ${ }^{69}$, M. Ouchrif ${ }^{136 d}$, E. A. Ouellette ${ }^{170}$, F. Ould-Saada ${ }^{118}$, A. Ouraou ${ }^{137}$, K. P. Oussoren ${ }^{106}$, Q. Ouyang ${ }^{33 a}$, A. Ovcharova ${ }^{15}$, M. Owen ${ }^{83}$, V. E. Ozcan ${ }^{19 a}$, N. Ozturk ${ }^{8}$, K. Pachal ${ }^{119}$, A. Pacheco Pages ${ }^{12}$, C. Padilla Aranda ${ }^{12}$, M. Pagáčováá ${ }^{48}$, S. Pagan Griso ${ }^{15}$, E. Paganis ${ }^{140}$, C. Pahl ${ }^{100}$, F. Paige ${ }^{25}$, P. Pais ${ }^{85}$, K. Pajchel ${ }^{118}$, G. Palacino ${ }^{160 b}$, S. Palestini ${ }^{30}$, M. Palka $^{38 b}$, D. Pallin ${ }^{34}$, A. Palma ${ }^{125 a, 125 b}$, J. D. Palmer ${ }^{18}$, Y. B. Pan ${ }^{174}$, E. Panagiotopoulou ${ }^{10}$, J. G. Panduro Vazquez ${ }^{76}$, P. Pani ${ }^{106}$, N. Panikashvili ${ }^{88}$, S. Panitkin ${ }^{25}$, D. Pantea ${ }^{26 a}$, L. Paolozzi ${ }^{134 a, 134 b}$, Th. D. Papadopoulou ${ }^{10}$, K. Papageorgiou ${ }^{155,1}$, A. Paramonov ${ }^{6}$, D. Paredes Hernandez ${ }^{34}$, M. A. Parker ${ }^{28}$, F. Parodi ${ }^{50 a, 50 b}$, J. A. Parsons ${ }^{35}$, U. Parzefall ${ }^{48}$, E. Pasqualucci ${ }^{133 a}$, S. Passaggio ${ }^{50 a}$, A. Passeri ${ }^{135 a}$, F. Pastore ${ }^{135 a, 135 b, *}$, Fr. Pastore ${ }^{76}$, G. Pásztor ${ }^{29}$, S. Pataraia ${ }^{176}$, N. D. Patel ${ }^{151}$, J. R. Pater ${ }^{83}$, S. Patricelli ${ }^{103 a, 103 b}$, T. Pauly ${ }^{30}$, J. Pearce ${ }^{170}$, L. E. Pedersen ${ }^{36}$, M. Pedersen ${ }^{118}$, S. Pedraza Lopez ${ }^{168}$, R. Pedro ${ }^{125 a, 125 b}$, S. V. Peleganchuk ${ }^{108}$, D. Pelikan ${ }^{167}$, H. Peng ${ }^{33 b}$, B. Penning ${ }^{31}$, J. Penwell ${ }^{60}$, D. V. Perepelitsa ${ }^{25}$, E. Perez Codina ${ }^{160 a}$, M. T. Pérez García-Estañ ${ }^{168}$, V. Perez Reale ${ }^{35}$, L. Perini ${ }^{90 a}$,90b, H. Pernegger ${ }^{30}$, R. Perrino ${ }^{72 a}$, R. Peschke ${ }^{42}$, V. D. Peshekhonov ${ }^{64}$, K. Peters ${ }^{30}$, R. F. Y. Peters ${ }^{83}$, B. A. Petersen ${ }^{30}$, T. C. Petersen ${ }^{36}$, E. Petit ${ }^{42}$, A. Petridis ${ }^{147,147 b}$, C. Petridou ${ }^{155}$, E. Petrolo ${ }^{133 a}$, F. Petrucci ${ }^{135 a, 135 b}$, N. E. Pettersson ${ }^{158}$, R. Pezoa ${ }^{32 b}$, P. W. Phillips ${ }^{130}$, G. Piacquadio ${ }^{144}$, E. Pianori ${ }^{171}, \quad$ A. Picazio ${ }^{49}$, E. Piccaro ${ }^{75}, \quad$ M. Piccinini20a,20b, R. Piegaia ${ }^{27}$, D. T. Pignotti ${ }^{110}$, J. E. Pilcher ${ }^{31}$, A. D. Pilkington ${ }^{77}, \quad$ J. Pina ${ }^{125 a, 125 b, 125 d}, \quad$ M. Pinamonti ${ }^{165 a, 165 c, a c}, \quad$ A. Pinder ${ }^{119}$, J. L. Pinfold ${ }^{3}$, A. Pingel ${ }^{36}$, B. Pinto ${ }^{125 a}$, S. Pires ${ }^{79}$, M. Pitt ${ }^{173}$, C. Pizio ${ }^{90 a}$,90b, L. Plazak ${ }^{145 a}$, M.-A. Pleier ${ }^{25}$, V. Pleskot ${ }^{128}$, E. Plotnikova ${ }^{64}$, P. Plucinski ${ }^{147 a, 147 b}$, S. Poddar ${ }^{58 a}$, F. Podlyski ${ }^{34}$, R. Poettgen ${ }^{82}$, L. Poggioli1 ${ }^{116}$, D. Pohl ${ }^{21}$, M. Pohl ${ }^{49}$, G. Polesello ${ }^{120 a}$, A. Policicchio $37 \mathrm{a}, 37 \mathrm{~b}$, R. Polifka ${ }^{159}$, A. Polini ${ }^{20 a}$, C. S. Pollard ${ }^{45}$, V. Polychronakos ${ }^{25}$, K. Pommès ${ }^{30}$, L. Pontecorvo ${ }^{133 a}$, B. G. Pope $^{89}$, G. A. Popeneciu' ${ }^{26 b}$, D. S. Popovic ${ }^{13 a}$, A. Poppleton ${ }^{30}$, X. Portell Bueso ${ }^{12}$, S. Pospisil ${ }^{127}$, K. Potamianos ${ }^{15}$, I. N. Potrap ${ }^{64}$, C. J. Potter ${ }^{150}$, C. T. Potter ${ }^{115}$, G. Poulard ${ }^{30}$, J. Poveda ${ }^{60}$, V. Pozdnyakov ${ }^{64}$, P. Pralavorio ${ }^{84}$, A. Pranko ${ }^{15}$, S. Prasad $^{30}$, R. Pravahan $^{8}$, S. Prell ${ }^{63}$, D. Price ${ }^{83}$, J. Price ${ }^{73}$, L. E. Price ${ }^{6}$, D. Prieur ${ }^{124}$, M. Primavera ${ }^{72 a}$, M. Proiss ${ }^{46}$, K. Prokofiev ${ }^{47}$, F. Prokoshin ${ }^{32 b}$, E. Protopapadaki ${ }^{137}$, S. Protopopescu ${ }^{25}$, J. Proudfoot ${ }^{6}$, M. Przybycien ${ }^{38 a}$, H. Przysiezniak ${ }^{5}$, E. Ptacek ${ }^{115}$, D. Puddu135a,135b, E. Pueschel ${ }^{85}$, D. Puldon ${ }^{149}$, M. Purohit ${ }^{25, a d}$, P. Puzo ${ }^{116}$, J. Qian ${ }^{88}$, G. Qin ${ }^{53}$, Y. Qin ${ }^{83}$, A. Quadt ${ }^{54}$, D. R. Quarrie ${ }^{15}$, W. B. Quayle ${ }^{165 a, 165 b}$, M. Queitsch-Maitland ${ }^{83}$, D. Quilty ${ }^{53}$, A. Qureshi ${ }^{160 b}$, V. Radeka ${ }^{25}$, V. Radescu ${ }^{42}$, S. K. Radhakrishnan ${ }^{149}$, P. Radloff ${ }^{115}$, P. $\operatorname{Rados}^{87}$, F. Ragusa ${ }^{90 a, 90 b}$, G. Rahal ${ }^{179}$, S. Rajagopalan ${ }^{25}$, M. Rammensee ${ }^{30}$, A. S. Randle-Conde ${ }^{40}$, C. Rangel-Smith ${ }^{167}$, K. Rao ${ }^{164}$, F. Rauscher ${ }^{99}$, T. C. Rave ${ }^{48}$, T. Ravenscroft ${ }^{53}$, M. Raymond ${ }^{30}$, A. L. Read ${ }^{118}$, N. P. Readioff ${ }^{73}$, D. M. Rebuzzi ${ }^{120 a, 120 b}$, A. Redelbach ${ }^{175}$, G. Redlinger ${ }^{25}$, R. Reece ${ }^{138}$, K. Reeves ${ }^{41}$, L. Rehnisch ${ }^{16}$, H. Reisin ${ }^{27}$, M. Relich ${ }^{164}$, C. Rembser ${ }^{30}$, H. Ren ${ }^{33 a}$, Z. L. Ren ${ }^{152}$, A. Renaud ${ }^{116}$, M. Rescigno ${ }^{133 a}$, S. Resconi ${ }^{90 a}$, O. L. Rezanova ${ }^{108, t}$, P. Reznicek ${ }^{128}$, R. Rezvanii ${ }^{94}$, R. Richter ${ }^{100}$, M. Ridel ${ }^{79}$, P. Rieck ${ }^{16}$, J. Rieger ${ }^{54}$, M. Rijssenbeek ${ }^{149}$, A. Rimoldi ${ }^{120 a, 120 b}$, L. Rinaldi ${ }^{20 a}$, E. Ritsch ${ }^{61}$, I. Riu ${ }^{12}$, F. Rizatdinova ${ }^{113}$, E. Rizvi ${ }^{75}$, S. H. Robertson ${ }^{86, i}$, A. Robichaud-Veronneau ${ }^{86}$, D. Robinson ${ }^{28}$, J. E. M. Robinson ${ }^{83}$, A. Robson ${ }^{53}$, C. Roda ${ }^{123 a, 123 b}$, L. Rodrigues ${ }^{30}$ ， S. Roe ${ }^{30}$ ， O. Røhne ${ }^{118}$ ， S. Rolli ${ }^{162}$, A. Romaniouk ${ }^{97}, \quad$ M. Romano ${ }^{20 a, 20 b}$, E. Romero Adam ${ }^{168}$,

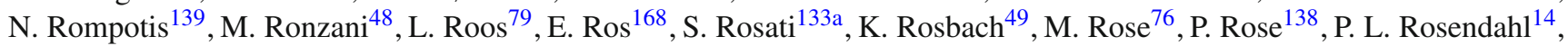
O. Rosenthal ${ }^{142}$ ， V. Rossetti ${ }^{147 a, 147 b}$ ， E. Rossi ${ }^{103 a, 103 b}, \quad$ L. P. Rossi ${ }^{50 a}, \quad$ R. Rosten ${ }^{139}$ ， M. Rotaru ${ }^{26 a}, \quad$ I. Roth ${ }^{173}$, J. Rothberg ${ }^{139}$, D. Rousseau ${ }^{116}$, C. R. Royon ${ }^{137}$, A. Rozanov ${ }^{84}$, Y. Rozen ${ }^{153}$, X. Ruan ${ }^{146 c}$, F. Rubbo ${ }^{12}$, I. Rubinskiy ${ }^{42}$, 


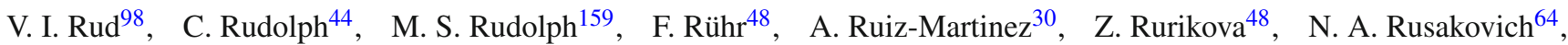
A. Ruschke ${ }^{99}$, J. P. Rutherfoord ${ }^{7}$, N. Ruthmann ${ }^{48}$, Y. F. Ryabov ${ }^{122}$, M. Rybar ${ }^{128}$, G. Rybkin ${ }^{116}$, N. C. Ryder ${ }^{119}$, A. F. Saavedra ${ }^{151}$, S. Sacerdoti ${ }^{27}$, A. Saddique ${ }^{3}$, I. Sadeh ${ }^{154}$, H. F.-W. Sadrozinski ${ }^{138}$, R. Sadykov ${ }^{64}$, F. Safai Tehrani ${ }^{133 a}$, H. Sakamoto ${ }^{156}$, Y. Sakurai ${ }^{172}$, G. Salamanna ${ }^{135 a, 135 b}$, A. Salamon ${ }^{134 a}$, M. Saleem ${ }^{112}$, D. Salek ${ }^{106}$, P. H. Sales De Bruin ${ }^{139}$, D. Salihagic ${ }^{100}$, A. Salnikov ${ }^{144}$, J. Salt ${ }^{168}$, D. Salvatore ${ }^{37 a, 37 b}$, F. Salvatore ${ }^{150}$, A. Salvucci ${ }^{105}, \quad$ A. Salzburger ${ }^{30}$, D. Sampsonidis ${ }^{155}$, A. Sanchez 103a,103b, J. Sánchez ${ }^{168}$, V. Sanchez Martinez ${ }^{168}$, H. Sandaker ${ }^{14}$, R. L. Sandbach ${ }^{75}$, H. G. Sander ${ }^{82}$, M. P. Sanders ${ }^{99}$, M. Sandhoff ${ }^{176}$, T. Sandoval ${ }^{28}$, C. Sandoval ${ }^{163}$, R. Sandstroem ${ }^{100}$, D. P. C. Sankey ${ }^{130}$, A. Sansoni ${ }^{47}$ ， C. Santoni ${ }^{34}$ ， R. Santonico ${ }^{134 a, 134 b}$, H. Santos ${ }^{125 a}$ ， I. Santoyo Castillo ${ }^{150}$ ， K. Sapp ${ }^{124}$, A. Sapronov ${ }^{64}$, J. G. Saraiva ${ }^{125 a, 125 d}$, B. Sarrazin ${ }^{21}$, G. Sartisohn ${ }^{176}$, O. Sasaki ${ }^{65}$, Y. Sasaki ${ }^{156}$, G. Sauvage ${ }^{5, *}$, E. Sauvan ${ }^{5}$, P. Savard ${ }^{159, d}$, D. O. Savu ${ }^{30}$ ， C. Sawyer ${ }^{119}$ ， L. Sawyer ${ }^{78, m}$ ， D. H. Saxon ${ }^{53}$ ， J. Saxon ${ }^{121}$ ， C. Sbarra ${ }^{20 a}$ ， A. Sbrizzi ${ }^{3} ，$ T. Scanlon ${ }^{77}$, D. A. Scannicchio ${ }^{164}$, M. Scarcella ${ }^{151}$, V. Scarfone ${ }^{37 a, 37 b}$, J. Schaarschmidt ${ }^{173}$, P. Schacht ${ }^{100}$, D. Schaefer ${ }^{30}$, R. Schaefer ${ }^{42}$, S. Schaepe ${ }^{21}$ ， S. Schaetzel ${ }^{58 b}$ ， U. Schäfer ${ }^{82}$ ， A. C. Schaffer ${ }^{116}$ ，D. Schaile ${ }^{99}$ ， R. D. Schamberger ${ }^{149}$ ， V. Scharf ${ }^{58 a}$, V. A. Schegelsky ${ }^{122}$ ，D. Scheirich ${ }^{128}$, M. Schernau ${ }^{164}$, M. I. Scherzer ${ }^{35}$ ，C. Schiavi50a,50b，J. Schieck ${ }^{99}$ ，C. Schillo ${ }^{48}$, M. Schioppa ${ }^{37 a, 37 b}$, S. Schlenker ${ }^{30}$, E. Schmidt ${ }^{48}$, K. Schmieden ${ }^{30}$, C. Schmitt ${ }^{82}$, S. Schmitt ${ }^{58 b}$, B. Schneider ${ }^{17}$, Y. J. Schnellbach ${ }^{73}$, U. Schnoor ${ }^{44}$, L. Schoeffel ${ }^{137}$, A. Schoening ${ }^{58 b}$, B. D. Schoenrock ${ }^{89}$, A. L. S. Schorlemmer ${ }^{54}$,

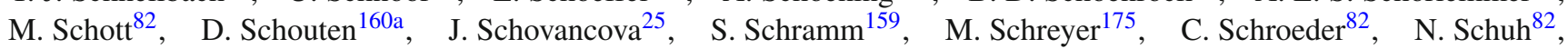

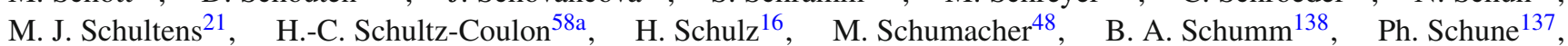
C. Schwanenberger ${ }^{83}$, A. Schwartzman ${ }^{144}$, Ph. Schwegler ${ }^{100}$, Ph. Schwemling ${ }^{137}$, R. Schwienhorst ${ }^{89}$, J. Schwindling ${ }^{137}$, T. Schwindt ${ }^{21}$, M. Schwoerer ${ }^{5}$, F. G. Sciacca ${ }^{17}$, E. Scifo ${ }^{116}$, G. Sciolla ${ }^{23}$, W. G. Scott ${ }^{130}$, F. Scuri ${ }^{123 a, 123 b}$, F. Scutti ${ }^{21}$, J. Searcy ${ }^{88}$, G. Sedov ${ }^{42}$, E. Sedykh ${ }^{122}$, S. C. Seidel ${ }^{104}$, A. Seiden ${ }^{138}$, F. Seifert ${ }^{127}$, J. M. Seixas ${ }^{24 a}$, G. Sekhniaidze ${ }^{103 a}$, S. J. Sekula ${ }^{40}$, K. E. Selbach ${ }^{46}$, D. M. Seliverstov ${ }^{122, *}$, G. Sellers ${ }^{73}$, N. Semprini-Cesari20a,20b , C. Serfon ${ }^{30}$, L. Serin ${ }^{116}$, L. Serkin ${ }^{54}$, T. Serre ${ }^{84}$, R. Seuster ${ }^{160 a}$, H. Severini ${ }^{112}$, T. Sfiligoj ${ }^{74}$, F. Sforza ${ }^{100}$, A. Sfyrla ${ }^{30}$, E. Shabalina ${ }^{54}$, M. Shamim ${ }^{115}$, L. Y. Shan ${ }^{33 a}$, R. Shang ${ }^{166}$, J. T. Shank ${ }^{22}$, M. Shapiro ${ }^{15}$, P. B. Shatalov ${ }^{96}$, K. Shaw ${ }^{165 a, 165 b}$, C. Y. Shehu ${ }^{150}$, P. Sherwood ${ }^{77}$, L. Shi ${ }^{152, \text { ae }}$ S. Shimizu 66 , C. O. Shimmin ${ }^{164}$, M. Shimojima ${ }^{101}$, M. Shiyakova ${ }^{64}$, A. Shmeleva95, M. J. Shochet ${ }^{31}$, D. Short ${ }^{119}$, S. Shrestha ${ }^{63}$, E. Shulga ${ }^{97}$, M. A. Shupe ${ }^{7}$, S. Shushkevich ${ }^{42}$, P. Sicho ${ }^{126}$, O. Sidiropoulou ${ }^{155}$, D. Sidorov ${ }^{113}$, A. Sidoti ${ }^{133 a}$, F. Siegert ${ }^{44}$, Dj. Sijacki ${ }^{13 a}$, J. Silva ${ }^{125 a, 125 d}$, Y. Silver ${ }^{154}, \quad$ D. Silverstein ${ }^{144}$, S. B. Silverstein ${ }^{147 a}$, V. Simak ${ }^{127}$, O. Simard ${ }^{5}$, Lj. Simic ${ }^{13 a}$, S. Simion ${ }^{116}$, E. Simioni ${ }^{82}$, B. Simmons ${ }^{77}$, R. Simoniello ${ }^{90 a}$,90b, M. Simonyan ${ }^{36}$, P. Sinervo ${ }^{159}$ ， N. B. Sinev ${ }^{115}$ ， V. Sipica ${ }^{142}$ ， G. Siragusa ${ }^{175}$ ， A. Sircar ${ }^{78}$ ， A. N. Sisakyan ${ }^{64, *}$, S. Yu. Sivoklokov ${ }^{98}$, J. Sjölin ${ }^{147 a, 147 b}$, T. B. Sjursen ${ }^{14}$, H. P. Skottowe ${ }^{57}$, K. Yu. Skovpen ${ }^{108}$, P. Skubic ${ }^{112}$, M. Slater ${ }^{18}$, T. Slavicek ${ }^{127}$, K. Sliwa ${ }^{162}$, V. Smakhtin ${ }^{173}$, B. H. Smart ${ }^{46}$, L. Smestad ${ }^{14}$, S. Yu. Smirnov ${ }^{97}$, Y. Smirnov ${ }^{97}$, L. N. Smirnova ${ }^{98, a f}$, O. Smirnova ${ }^{80}$, K. M. Smith ${ }^{53}$, M. Smizanska ${ }^{71}$, K. Smolek ${ }^{127}$, A. A. Snesarev ${ }^{95}$, G. Snidero ${ }^{75}$, S. Snyder ${ }^{25}$, R. Sobie ${ }^{170, i}$, F. Socher ${ }^{44}$, A. Soffer ${ }^{154}$, D. A. Soh ${ }^{152, a e, ~ C . ~ A . ~ S o l a n s ~}{ }^{30}$, M. Solar ${ }^{127}$, J. Solc ${ }^{127}$, E. Yu. Soldatov ${ }^{97}$, U. Soldevila ${ }^{168}$, A. A. Solodkov ${ }^{129}$, A. Soloshenko ${ }^{64}$, O. V. Solovyanov ${ }^{129}$, V. Solovyev ${ }^{122}$, P. Sommer ${ }^{48}$, H. Y. Song ${ }^{33 b}$, N. Soni ${ }^{1}$, A. Sood ${ }^{15}$, A. Sopczak ${ }^{127}$, B. Sopko ${ }^{127}$, V. Sopko ${ }^{127}$, V. Sorin ${ }^{12}$, M. Sosebee ${ }^{8}$, R. Soualah ${ }^{165 a, 165 c}$, P. Soueid ${ }^{94}$, A. M. Soukharev ${ }^{108}$, D. South ${ }^{42}$, S. Spagnolo ${ }^{72 a, 72 b}$, F. Spanò ${ }^{76}$, W. R. Spearman ${ }^{57}$, F. Spettel ${ }^{100}$, R. Spighi ${ }^{20 a}$, G. Spigo ${ }^{30}$, L. A. Spiller ${ }^{87}$, M. Spousta ${ }^{128}$, T. Spreitzer ${ }^{159}$, B. Spurlock ${ }^{8}$, R. D. St. Denis ${ }^{53}$,* S. Staerz ${ }^{44}$, J. Stahlman ${ }^{121}$, R. Stamen ${ }^{58 a}$, S. Stamm ${ }^{16}$, E. Stanecka ${ }^{39}$, R. W. Stanek ${ }^{6}$, C. Stanescu ${ }^{135 a}$, M. Stanescu-Bellu ${ }^{42}$, M. M. Stanitzki ${ }^{42}$, S. Stapnes ${ }^{118}$, E. A. Starchenko ${ }^{129}$ ， J. Stark ${ }^{55}$ ， P. Staroba ${ }^{126}$ ， P. Starovoitov ${ }^{42}$ ， R. Staszewski ${ }^{39}$ ， P. Stavina ${ }^{145 a, *}$, $\begin{array}{lll}\text { P. Steinberg } & 25\end{array}$ B. Stelzer ${ }^{143}$, H. J. Stelzer ${ }^{30}$ ， O. Stelzer-Chilton ${ }^{160 a}$ ， H. Stenzel ${ }^{52}$ ， S. Stern ${ }^{100 ， \quad \text { G. A. Stewart }}{ }^{53}$, J. A. Stillings ${ }^{21}$, M. C. Stockton ${ }^{86}$, M. Stoebe ${ }^{86}$, G. Stoicea ${ }^{26 a}$, P. Stolte ${ }^{54}$, S. Stonjek ${ }^{100}$, A. R. Stradling ${ }^{8}$, A. Straessner ${ }^{44}$, M. E. Stramaglia ${ }^{17}$, J. Strandberg ${ }^{148}$, S. Strandberg ${ }^{147 a, 147 b}$, A. Strandlie ${ }^{118}$, E. Strauss ${ }^{144}$, M. Strauss ${ }^{112}$, P. Strizenec ${ }^{145 b}$, R. Ströhmer ${ }^{175}$, D. M. Strom ${ }^{115}$, R. Stroynowski ${ }^{40}$, A. Struebig ${ }^{105}$, S. A. Stucci ${ }^{17}$, B. Stugu ${ }^{14}$, N. A. Styles ${ }^{42}$, D. Su ${ }^{144}$, J. Su ${ }^{124}$, R. Subramaniam ${ }^{78}$, A. Succurro ${ }^{12}$, Y. Sugaya ${ }^{117}$, C. Suhr ${ }^{107}$, M. Suk ${ }^{127}$, V. V. Sulin ${ }^{95}$, S. Sultansoy ${ }^{4 c}$, T. Sumida ${ }^{67}$, S. Sun ${ }^{57}$, X. Sun ${ }^{33 a}$, J. E. Sundermann ${ }^{48}$, K. Suruliz ${ }^{140}$, G. Susinno ${ }^{37 a, 37 b}$, M. R. Sutton ${ }^{150}$, Y. Suzuki ${ }^{65}$, M. Svatos ${ }^{126}$, S. Swedish ${ }^{169}$, M. Swiatlowski ${ }^{144}$, I. Sykora ${ }^{145 a}$, T. Sykora ${ }^{128}$, D. Ta ${ }^{89}$, C. Taccini ${ }^{135 a, 135 b}$, K. Tackmann ${ }^{42}$, J. Taenzer ${ }^{159}$, A. Taffard ${ }^{164}$, R. Tafirout ${ }^{160 a}$, N. Taiblum ${ }^{154}$, H. Takai ${ }^{25}$, R. Takashima ${ }^{68}$, H. Takeda ${ }^{66}$, T. Takeshita ${ }^{141}$, Y. Takubo ${ }^{65}$, M. Talby ${ }^{84}$, A. A. Talyshev ${ }^{108, t}$, J. Y. C. Tam ${ }^{175}$, K. G. Tan ${ }^{87}$, J. Tanaka ${ }^{156}$, R. Tanaka ${ }^{116}$, S. Tanaka ${ }^{132}$, S. Tanaka ${ }^{65}$, A. J. Tanasijczuk ${ }^{143}$, B. B. Tannenwald ${ }^{110}$, N. Tannoury ${ }^{21}$, S. Tapprogge ${ }^{82}$, S. Tarem ${ }^{153}$, F. Tarrade ${ }^{29}$, G. F. Tartarelli ${ }^{90 a}$, P. Tas ${ }^{128}$, M. Tasevsky ${ }^{126}$, T. Tashiro ${ }^{67}$, E. Tassi ${ }^{37 a, 37 b}$, A. Tavares Delgado ${ }^{125 a, 125 b}$, Y. Tayalati ${ }^{136 d}$, F. E. Taylor ${ }^{93}$, G. N. Taylor ${ }^{87}$, W. Taylor ${ }^{160 b}$, F. A. Teischinger ${ }^{30}$, M. Teixeira Dias Castanheira ${ }^{75}$, P. Teixeira-Dias ${ }^{76}$, K. K. Temming ${ }^{48}$, H. Ten Kate ${ }^{30}$, P. K. Teng ${ }^{152}$, J. J. Teoh ${ }^{117}, \quad$ S. Terada ${ }^{65}, \quad$ K. Terashi ${ }^{156}, \quad$ J. Terron ${ }^{81}, \quad$ S. Terzo ${ }^{100,}$ M. Testa ${ }^{47}$, R. J. Teuscher ${ }^{159, \mathrm{i}}$, J. Therhaag ${ }^{21}$ ， T. Theveneaux-Pelzer ${ }^{34}$, J. P. Thomas ${ }^{18}$, J. Thomas-Wilsker ${ }^{76}$, E. N. Thompson ${ }^{35}$,

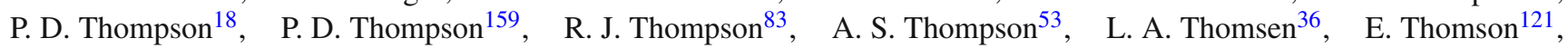


M. Thomson ${ }^{28}$, W. M. Thong ${ }^{87}$, R. P. Thun ${ }^{88, *}$, F. $\operatorname{Tian}^{35}$, M. J. Tibbetts ${ }^{15}$, V. O. Tikhomirov ${ }^{95, a g}$, Yu. A. Tikhonov ${ }^{108, t}$, S. Timoshenko ${ }^{97}$, E. Tiouchichine ${ }^{84}$, P. Tipton ${ }^{177}$, S. Tisserant ${ }^{84}$, T. Todorov ${ }^{5}$, S. Todorova-Nova ${ }^{128}$, B. Toggerson ${ }^{7}$, J. Tojo ${ }^{69}$, S. Tokár ${ }^{145 a}$, K. Tokushuku ${ }^{65}$, K. Tollefson ${ }^{89}$, L. Tomlinson ${ }^{83}$, M. Tomoto ${ }^{102}$, L. Tompkins ${ }^{31}$, K. Toms ${ }^{104}$, N. D. Topilin ${ }^{64}$, E. Torrence ${ }^{115}$, H. Torres ${ }^{143}$, E. Torró Pastor ${ }^{168}$, J. Toth ${ }^{84, \text { ah }}$, F. Touchard ${ }^{84}$, D. R. Tovey ${ }^{140}$, H. L. Tran ${ }^{116}$, T. Trefzger ${ }^{175}$, L. Tremblet ${ }^{30}$, A. Tricoli ${ }^{30}$, I. M. Trigger ${ }^{160 a}$, S. Trincaz-Duvoid ${ }^{79}$, M. F. Tripiana ${ }^{12}$, W. Trischuk ${ }^{159}$, B. Trocmé ${ }^{55}$, C. Troncon ${ }^{90 a}$, M. Trottier-McDonald ${ }^{143}$, M. Trovatelli135a,135b, P. True ${ }^{89}$, M. Trzebinski ${ }^{39}$, A. Trzupek ${ }^{39}$, C. Tsarouchas $^{30}$, J. C.-L. Tseng ${ }^{119}$, P. V. Tsiareshka ${ }^{91}$, D. Tsionou ${ }^{137}$, G. Tsipolitis ${ }^{10}$, N. Tsirintanis ${ }^{9}$, S. Tsiskaridze ${ }^{12}$, V. Tsiskaridze ${ }^{48}$, E. G. Tskhadadze ${ }^{51 \mathrm{a}}$, I. I. Tsukerman ${ }^{96}$, V. Tsulaia ${ }^{15}$, S. Tsuno ${ }^{65}$, D. Tsybychev ${ }^{149}$, A. Tudorache ${ }^{26 a}$, V. Tudorache ${ }^{26 a}$, A. N. Tuna ${ }^{121}$, S. A. Tupputi ${ }^{20 a, 20 b}$, S. Turchikhin ${ }^{98, a f}$, D. Turecek ${ }^{127}$, I. Turk Cakir ${ }^{4 d}$, R. Turra ${ }^{90 a, 90 b}$, P. M. Tuts ${ }^{35}$, A. Tykhonov ${ }^{49}$, M. Tylmad ${ }^{147 a, 147 b}$, M. Tyndel ${ }^{130}$, K. Uchida ${ }^{21}$, I. Ueda ${ }^{156}$, R. Ueno ${ }^{29}$, M. Ughetto ${ }^{84}$, M. Ugland ${ }^{14}$, M. Uhlenbrock ${ }^{21}$, F. Ukegawa ${ }^{161}$, G. Unal ${ }^{30}$, A. Undrus ${ }^{25}$, G. Unel ${ }^{164}$, F. C. Ungaro ${ }^{48}$, Y. Unno ${ }^{65}$, C. Unverdorben ${ }^{99}$, D. Urbaniec ${ }^{35}$, P. Urquijo ${ }^{87}$, G. Usai ${ }^{8}$, A. Usanova ${ }^{61}$, L. Vacavant ${ }^{84}$, V. Vacek ${ }^{127}$, B. Vachon ${ }^{86}$, N. Valencic ${ }^{106}$, S. Valentinetti ${ }^{20 a}, 20 \mathrm{~b}$, A. Valero ${ }^{168}$, L. Valery ${ }^{34}$, S. Valkar ${ }^{128}$, E. Valladolid Gallego ${ }^{168}$, S. Vallecorsa ${ }^{49}$, $\begin{array}{ll}\text { J. A. Valls Ferrer } & 168\end{array}$ W. Van Den Wollenberg ${ }^{106}$, P. C. Van Der Deijl ${ }^{106}, \quad$ R. van der Geer ${ }^{106}$, H. van der Graaf ${ }^{106}$, R. Van Der Leeuw ${ }^{106}$, D. van der $\operatorname{Ster}^{30}$, N. van $\operatorname{Eldik}^{30}$, P. van Gemmeren ${ }^{6}$, J. Van Nieuwkoop ${ }^{143}$, I. van Vulpen ${ }^{106}$, M. C. van Woerden $^{30}$, M. Vanadia ${ }^{133 a}$,133b , W. Vandelli ${ }^{30}$, R. Vanguri ${ }^{121}$, A. Vaniachine ${ }^{6}$, P. Vankov ${ }^{42}$, F. Vannucci ${ }^{79}$, G. Vardanyan ${ }^{178}$, R. Vari ${ }^{133 a}$, E. W. Varnes ${ }^{7}$, T. $\operatorname{Varol}^{85}$, D. Varouchas ${ }^{79}$, A. Vartapetian ${ }^{8}$, K. E. Varvell ${ }^{151}$, F. Vazeille ${ }^{34}$, T. Vazquez Schroeder ${ }^{54}$, J. Veatch ${ }^{7}$, F. Veloso ${ }^{125 a, 125 c}$, S. Veneziano ${ }^{133 a}$, A. Ventura ${ }^{72 a, 72 b}$, D. Ventura ${ }^{85}$, M. Venturi ${ }^{170}$, N. Venturi ${ }^{159}$, A. Venturini23 ${ }^{23}$ V. Vercesi ${ }^{120 a}$, M. Verducci ${ }^{133 a}, 133 b$, W. Verkerke ${ }^{106}$, J. C. Vermeulen ${ }^{106}$, A. Vest ${ }^{44}$, M. C. Vetterli ${ }^{143, d}$, O. Viazlo ${ }^{80}$, I. Vichou ${ }^{166}$, T. Vickey ${ }^{146 c, a i}$, O. E. Vickey Boeriu ${ }^{146 c}$, G. H. A. Viehhauser ${ }^{19}{ }^{19}$, S. Viel $^{169}$, R. Vigne ${ }^{30}$, M. Villa ${ }^{20 a, 20 b}$, M. Villaplana Perez ${ }^{90 a, 90 b}$, E. Vilucchi ${ }^{47}$, M. G. Vincter ${ }^{29}$, V. B. Vinogradov ${ }^{64}$, J. Virzi ${ }^{15}$,

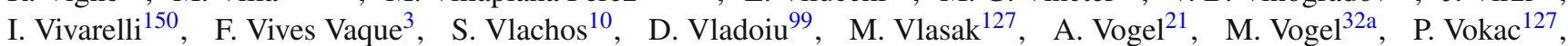
G. Volpi ${ }^{123 a}$,123b, M. Volpi ${ }^{87}$, H. von der Schmitt ${ }^{100}$, H. von Radziewski ${ }^{48}$, E. von Toerne ${ }^{21}$, V. Vorobel ${ }^{128}$, K. Vorobev ${ }^{97}$, M. Vos ${ }^{168}$, R. $\operatorname{Voss}^{30}$, J. H. Vossebeld ${ }^{73}$, N. Vranjes ${ }^{137}$, M. Vranjes Milosavljevic ${ }^{13 a}$, V. Vrba ${ }^{126}$, M. Vreeswijk ${ }^{106}$, T. Vu Anh ${ }^{48}$, R. Vuillermet ${ }^{30}$, I. Vukotic ${ }^{31}$, Z. Vykydal ${ }^{127}$, P. Wagner ${ }^{21}$, W. Wagner ${ }^{176}$, H. Wahlberg ${ }^{70}$, S. Wahrmund ${ }^{44}$,

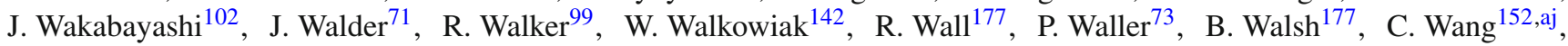
C. Wang ${ }^{45}$, F. Wang ${ }^{174}$, H. Wang ${ }^{15}$, H. Wang ${ }^{40}$, J. Wang ${ }^{42}$, J. Wang ${ }^{33 a}$, K. Wang ${ }^{86}$, R. Wang ${ }^{104}$, S. M. Wang ${ }^{152}$,

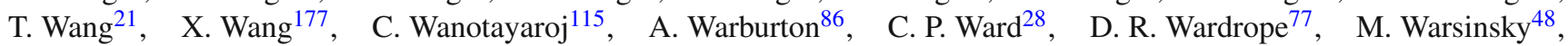
A. Washbrook ${ }^{46}$, C. Wasicki ${ }^{42}$, P. M. Watkins ${ }^{18}$, A. T. Watson ${ }^{18}$, I. J. Watson ${ }^{151}$, M. F. Watson ${ }^{18}$, G. Watts ${ }^{139}$, S. Watts ${ }^{83}$, B. M. Waugh ${ }^{77}$, S. Webb ${ }^{83}$, M. S. Weber ${ }^{17}$, S. W. Weber ${ }^{175}$, J. S. Webster ${ }^{31}$, A. R. Weidberg ${ }^{119}$, P. Weigell ${ }^{100}$, B. Weinert ${ }^{60}$, J. Weingarten ${ }^{54}$, C. Weiser ${ }^{48}$, H. Weits ${ }^{106}$, P. S. Wells ${ }^{30}$, T. Wenaus ${ }^{25}$, D. Wendland ${ }^{16}$, Z. Weng ${ }^{152, a e}$, T. Wengler ${ }^{30}$, S. Wenig ${ }^{30}$, N. Wermes ${ }^{21}$, M. Werner ${ }^{48}$, P. Werner ${ }^{30}$, M. Wessels ${ }^{58 a}$, J. Wetter ${ }^{162}$, K. Whalen ${ }^{29}$, A. White ${ }^{8}$, M. J. White ${ }^{1}$, R. White ${ }^{32 b}$ ， S. White ${ }^{123 a, 123 b}$, D. Whiteson ${ }^{164}$, D. Wicke ${ }^{176}$, F. J. Wickens ${ }^{130}$, W. Wiedenmann ${ }^{174}$,

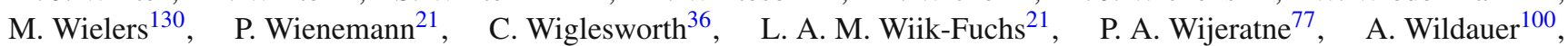
M. A. Wildt ${ }^{42, a k}$, H. G. Wilkens ${ }^{30}$, J. Z. Will ${ }^{99}$, H. H. Williams ${ }^{121}$, S. Williams ${ }^{28}$, C. Willis ${ }^{89}$, S. Willocq ${ }^{85}$, A. Wilson ${ }^{88}$, J. A. Wilson ${ }^{18}$, I. Wingerter-Seez ${ }^{5}$, F. Winklmeier ${ }^{115}$, B. T. Winter ${ }^{21}$, M. Wittgen ${ }^{144}$, T. Wittig ${ }^{43}$, J. Wittkowski ${ }^{99}$, S. J. Wollstadt ${ }^{82}$, M. W. Wolter ${ }^{39}$, H. Wolters ${ }^{125 a, 125 c}$, B. K. Wosiek ${ }^{39}$, J. Wotschack ${ }^{30}$, M. J. Woudstra ${ }^{83}$, K. W. Wozniak ${ }^{39}$, M. Wright ${ }^{53}, \quad$ M. Wu ${ }^{55}$, S. L. Wu ${ }^{174}$, X. Wu ${ }^{49}$, Y. Wu ${ }^{88}$, E. Wulf ${ }^{35}$, T. R. Wyatt ${ }^{83}$, B. M. Wynne ${ }^{46}, \quad$ S. Xella ${ }^{36}$, M. Xiao ${ }^{137}$, D. Xu ${ }^{33 a}$, L. Xu ${ }^{33 b, a l}$, B. Yabsley ${ }^{151}$, S. Yacoob ${ }^{146 b, a m}$, R. Yakabe ${ }^{66}$, M. Yamada ${ }^{65}$, H. Yamaguchi ${ }^{156}$, Y. Yamaguchi ${ }^{117}$, A. Yamamoto ${ }^{65}$, K. Yamamoto ${ }^{63}$, S. Yamamoto ${ }^{156}$, T. Yamamura ${ }^{156}$, T. Yamanaka ${ }^{156}$, K. Yamauchi ${ }^{102}$, Y. Yamazaki ${ }^{66}$, Z. Yan ${ }^{22}$, H. Yang ${ }^{33 e}$, H. Yang ${ }^{174}$, U. K. Yang ${ }^{83}$, Y. Yang ${ }^{110}$, S. Yanush ${ }^{92}$, L. Yao ${ }^{33 a}$, W.-M. Yao ${ }^{15}$, Y. Yasu ${ }^{65}$, E. Yatsenko ${ }^{42}$, K. H. Yau Wong ${ }^{21}$, J. Ye ${ }^{40}$, S. Ye ${ }^{25}$, I. Yeletskikh ${ }^{64}$, A. L. Yen ${ }^{57}$, E. Yildirim ${ }^{42}$, M. Yilmaz ${ }^{4 b}$, R. Yoosoofmiya ${ }^{124}$, K. Yorita ${ }^{172}$, R. Yoshida ${ }^{6}$, K. Yoshihara ${ }^{156}$, C. Young ${ }^{144}$, C. J. S. Young ${ }^{30}$, S. Youssef ${ }^{22}$, D. R. Yu ${ }^{15}$, J. Yu ${ }^{8}$, J. M. Yu ${ }^{88}$, J. Yu ${ }^{113}$, L. Yuan ${ }^{66}$, A. Yurkewicz ${ }^{107}$, I. Yusuff ${ }^{28, \text { an }, \text { B. Zabinski }{ }^{39} \text {, R. Zaidan }}{ }^{62}$, A. M. Zaitsev ${ }^{129, a a,}$ A. Zaman ${ }^{149}$, S. Zambito ${ }^{23}$, L. Zanello133a,133b, D. Zanzi ${ }^{100}$, C. Zeitnitz ${ }^{176}$, M. Zeman ${ }^{127}$, A. Zemla ${ }^{38 a}$, K. Zengel ${ }^{23}$, O. Zenin ${ }^{129}$ ， T. Ženišs ${ }^{145 a}$ ， D. Zerwas ${ }^{116}$ ， G. Zevi della Porta ${ }^{57}$ ， D. Zhang ${ }^{88}$ ， F. Zhang ${ }^{174}$ ， H. Zhang ${ }^{89}$, J. Zhang ${ }^{6}$, L. Zhang ${ }^{152}$, X. Zhang ${ }^{33 d}$, Z. Zhang ${ }^{116}$, Z. Zhao ${ }^{33 b}$, A. Zhemchugov ${ }^{64}$, J. Zhong ${ }^{119}$, B. Zhou ${ }^{88}$, L. Zhou ${ }^{35}$, N. Zhou ${ }^{164}$, C. G. Zhu ${ }^{33 d}$, H. Zhu ${ }^{33 a}$, J. Zhu ${ }^{88}$, Y. Zhu ${ }^{33 b}$, X. Zhuang ${ }^{33 a}$, K. Zhukov ${ }^{95}$, A. Zibell ${ }^{175}$, D. Zieminska ${ }^{60}$, N. I. Zimine ${ }^{64}$, $\begin{array}{llll}\text { C. Zimmermann } & \text { 82, } & \text { R. Zimmermann } & \text { 21 }\end{array}$ S. Zimmermann ${ }^{21}, \quad$ S. Zimmermann ${ }^{48}, \quad$ Z. Zinonos ${ }^{54}, \quad$ M. Ziolkowski ${ }^{142}$, G. Zobernig ${ }^{174}$, A. Zoccoli ${ }^{20 a, 20 b}$, M. zur Nedden ${ }^{16}$, G. Zurzolo ${ }^{103 a, 103 b}$, V. Zutshi ${ }^{107}$, L. Zwalinski ${ }^{30}$

${ }^{1}$ Department of Physics, University of Adelaide, Adelaide, Australia

${ }^{2}$ Physics Department, SUNY Albany, Albany, NY, USA 
${ }^{3}$ Department of Physics, University of Alberta, Edmonton, AB, Canada

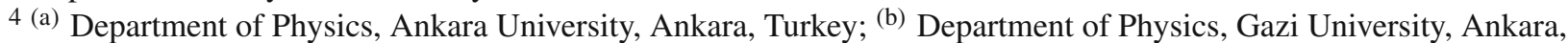

Turkey; ${ }^{(c)}$ Division of Physics, TOBB University of Economics and Technology, Ankara, Turkey; ${ }^{(d)}$ Turkish Atomic

Energy Authority, Ankara, Turkey

${ }^{5}$ LAPP, CNRS/IN2P3 and Université de Savoie, Annecy-le-Vieux, France

${ }^{6}$ High Energy Physics Division, Argonne National Laboratory, Argonne, IL, USA

${ }^{7}$ Department of Physics, University of Arizona, Tucson, AZ, USA

${ }^{8}$ Department of Physics, The University of Texas at Arlington, Arlington, TX, USA

${ }^{9}$ Physics Department, University of Athens, Athens, Greece

${ }^{10}$ Physics Department, National Technical University of Athens, Zografou, Greece

${ }^{11}$ Institute of Physics, Azerbaijan Academy of Sciences, Baku, Azerbaijan

${ }^{12}$ Institut de Física d'Altes Energies and Departament de Física de la Universitat Autònoma de Barcelona, Barcelona, Spain

13 (a) Institute of Physics, University of Belgrade, Belgrade, Serbia; (b) Vinca Institute of Nuclear Sciences, University of Belgrade, Belgrade, Serbia

${ }^{14}$ Department for Physics and Technology, University of Bergen, Bergen, Norway

15 Physics Division, Lawrence Berkeley National Laboratory and University of California, Berkeley, CA, USA

${ }^{16}$ Department of Physics, Humboldt University, Berlin, Germany

${ }^{17}$ Albert Einstein Center for Fundamental Physics and Laboratory for High Energy Physics, University of Bern, Bern, Switzerland

${ }^{18}$ School of Physics and Astronomy, University of Birmingham, Birmingham, UK

19 (a) Department of Physics, Bogazici University, Istanbul, Turkey; ${ }^{(b)}$ Department of Physics, Dogus University, Istanbul, Turkey; ${ }^{(c)}$ Department of Physics Engineering, Gaziantep University, Gaziantep, Turkey

20 (a) INFN Sezione di Bologna, Bologna, Italy; (b) Dipartimento di Fisica e Astronomia, Università di Bologna, Bologna, Italy

${ }^{21}$ Physikalisches Institut, University of Bonn, Bonn, Germany

22 Department of Physics, Boston University, Boston, MA, USA

${ }^{23}$ Department of Physics, Brandeis University, Waltham, MA, USA

24 (a) Universidade Federal do Rio De Janeiro COPPE/EE/IF, Rio de Janeiro, Brazil; (b) Federal University of Juiz de Fora (UFJF), Juiz de Fora, Brazil; (c) Federal University of Sao Joao del Rei (UFSJ), Sao Joao del Rei, Brazil; (d) Instituto de Fisica, Universidade de Sao Paulo, São Paulo, Brazil

${ }^{25}$ Physics Department, Brookhaven National Laboratory, Upton, NY, USA

26 (a) National Institute of Physics and Nuclear Engineering, Bucharest, Romania; (b) Physics Department, National

Institute for Research and Development of Isotopic and Molecular Technologies, Cluj Napoca, Romania; (c) University

Politehnica Bucharest, Bucharest, Romania; ${ }^{(d)}$ West University in Timisoara, Timisoara, Romania

${ }^{27}$ Departamento de Física, Universidad de Buenos Aires, Buenos Aires, Argentina

${ }^{28}$ Cavendish Laboratory, University of Cambridge, Cambridge, UK

${ }^{29}$ Department of Physics, Carleton University, Ottawa, ON, Canada

${ }^{30}$ CERN, Geneva, Switzerland

${ }^{31}$ Enrico Fermi Institute, University of Chicago, Chicago, IL, USA

32 (a) Departamento de Física, Pontificia Universidad Católica de Chile, Santiago, Chile; (b) Departamento de Física, Universidad Técnica Federico Santa María, Valparaiso, Chile

33 (a) Institute of High Energy Physics, Chinese Academy of Sciences, Beijing, China; (b) Department of Modern Physics, University of Science and Technology of China, Hefei, Anhui, China; ${ }^{(c)}$ Department of Physics, Nanjing University,

Nanjing, Jiangsu, China; (d) School of Physics, Shandong University, Jinan, Shandong, China; (e) Physics Department, Shanghai Jiao Tong University, Shanghai, China

${ }^{34}$ Laboratoire de Physique Corpusculaire, Clermont Université and Université Blaise Pascal and CNRS/IN2P3, Clermont-Ferrand, France

${ }^{35}$ Nevis Laboratory, Columbia University, Irvington, NY, USA

${ }^{36}$ Niels Bohr Institute, University of Copenhagen, Copenhagen, Denmark

37 (a) INFN Gruppo Collegato di Cosenza, Laboratori Nazionali di Frascati, Frascati, Italy; (b) Dipartimento di Fisica, Università della Calabria, Rende, Italy

38 (a) Faculty of Physics and Applied Computer Science, AGH University of Science and Technology, Kraków, Poland; 
(b) Marian Smoluchowski Institute of Physics, Jagiellonian University, Kraków, Poland

39 The Henryk Niewodniczanski Institute of Nuclear Physics, Polish Academy of Sciences, Kraków, Poland

${ }^{40}$ Physics Department, Southern Methodist University, Dallas, TX, USA

${ }^{41}$ Physics Department, University of Texas at Dallas, Richardson, TX, USA

42 DESY, Hamburg and Zeuthen, Germany

43 Institut für Experimentelle Physik IV, Technische Universität Dortmund, Dortmund, Germany

44 Institut für Kern- und Teilchenphysik, Technische Universität Dresden, Dresden, Germany

45 Department of Physics, Duke University, Durham, NC, USA

46 SUPA-School of Physics and Astronomy, University of Edinburgh, Edinburgh, UK

47 INFN Laboratori Nazionali di Frascati, Frascati, Italy

${ }^{48}$ Fakultät für Mathematik und Physik, Albert-Ludwigs-Universität, Freiburg, Germany

49 Section de Physique, Université de Genève, Geneva, Switzerland

50 (a) INFN Sezione di Genova, Genoa, Italy; (b) Dipartimento di Fisica, Università di Genova, Genova, Italy

51 (a) E. Andronikashvili Institute of Physics, Iv. Javakhishvili Tbilisi State University, Tbilisi, Georgia; ${ }^{(b)}$ High Energy Physics Institute, Tbilisi State University, Tbilisi, Georgia

52 II Physikalisches Institut, Justus-Liebig-Universität Giessen, Giessen, Germany

53 SUPA-School of Physics and Astronomy, University of Glasgow, Glasgow, UK

54 II Physikalisches Institut, Georg-August-Universität, Göttingen, Germany

55 Laboratoire de Physique Subatomique et de Cosmologie, Université Grenoble-Alpes, CNRS/IN2P3, Grenoble, France

56 Department of Physics, Hampton University, Hampton, VA, USA

57 Laboratory for Particle Physics and Cosmology, Harvard University, Cambridge, MA, USA

58 (a) Kirchhoff-Institut für Physik, Ruprecht-Karls-Universität Heidelberg, Heidelberg, Germany; ${ }^{\text {(b) }}$ Physikalisches Institut, Ruprecht-Karls-Universität Heidelberg, Heidelberg, Germany; ${ }^{(c)}$ ZITI Institut für technische Informatik, Ruprecht-Karls-Universität Heidelberg, Mannheim, Germany

${ }^{59}$ Faculty of Applied Information Science, Hiroshima Institute of Technology, Hiroshima, Japan

${ }^{60}$ Department of Physics, Indiana University, Bloomington, IN, USA

${ }^{61}$ Institut für Astro- und Teilchenphysik, Leopold-Franzens-Universität, Innsbruck, Austria

${ }^{62}$ University of Iowa, Iowa City, IA, USA

63 Department of Physics and Astronomy, Iowa State University, Ames, IA, USA

64 Joint Institute for Nuclear Research, JINR Dubna, Dubna, Russia

65 KEK, High Energy Accelerator Research Organization, Tsukuba, Japan

66 Graduate School of Science, Kobe University, Kobe, Japan

${ }^{67}$ Faculty of Science, Kyoto University, Kyoto, Japan

68 Kyoto University of Education, Kyoto, Japan

69 Department of Physics, Kyushu University, Fukuoka, Japan

${ }^{70}$ Instituto de Física La Plata, Universidad Nacional de La Plata and CONICET, La Plata, Argentina

71 Physics Department, Lancaster University, Lancaster, UK

72 (a) INFN Sezione di Lecce, Lecce, Italy; (b) Dipartimento di Matematica e Fisica, Università del Salento, Lecce, Italy

73 Oliver Lodge Laboratory, University of Liverpool, Liverpool, UK

74 Department of Physics, Jožef Stefan Institute and University of Ljubljana, Ljubljana, Slovenia

75 School of Physics and Astronomy, Queen Mary University of London, London, UK

76 Department of Physics, Royal Holloway University of London, Surrey, UK

77 Department of Physics and Astronomy, University College London, London, UK

78 Louisiana Tech University, Ruston, LA, USA

${ }^{79}$ Laboratoire de Physique Nucléaire et de Hautes Energies, UPMC and Université Paris-Diderot and CNRS/IN2P3, Paris, France

${ }^{80}$ Fysiska institutionen, Lunds universitet, Lund, Sweden

${ }^{81}$ Departamento de Fisica Teorica C-15, Universidad Autonoma de Madrid, Madrid, Spain

${ }^{82}$ Institut für Physik, Universität Mainz, Mainz, Germany

83 School of Physics and Astronomy, University of Manchester, Manchester, UK

${ }^{84}$ CPPM, Aix-Marseille Université and CNRS/IN2P3, Marseille, France

85 Department of Physics, University of Massachusetts, Amherst, MA, USA

${ }^{86}$ Department of Physics, McGill University, Montreal, QC, Canada 
${ }^{87}$ School of Physics, University of Melbourne, Parkville, VIC, Australia

${ }^{88}$ Department of Physics, The University of Michigan, Ann Arbor, MI, USA

${ }^{89}$ Department of Physics and Astronomy, Michigan State University, East Lansing, MI, USA

90 (a) INFN Sezione di Milano, Milan, Italy; (b) Dipartimento di Fisica, Università di Milano, Milan, Italy

${ }^{91}$ B.I. Stepanov Institute of Physics, National Academy of Sciences of Belarus, Minsk, Republic of Belarus

${ }^{92}$ National Scientific and Educational Centre for Particle and High Energy Physics, Minsk, Republic of Belarus

${ }^{93}$ Department of Physics, Massachusetts Institute of Technology, Cambridge, MA, USA

${ }^{94}$ Group of Particle Physics, University of Montreal, Montreal, QC, Canada

95 P.N. Lebedev Institute of Physics, Academy of Sciences, Moscow, Russia

${ }^{96}$ Institute for Theoretical and Experimental Physics (ITEP), Moscow, Russia

${ }^{97}$ Moscow Engineering and Physics Institute (MEPhI), Moscow, Russia

${ }^{98}$ D.V. Skobeltsyn Institute of Nuclear Physics, M.V. Lomonosov Moscow State University, Moscow, Russia

${ }^{99}$ Fakultät für Physik, Ludwig-Maximilians-Universität München, Munich, Germany

${ }^{100}$ Max-Planck-Institut für Physik (Werner-Heisenberg-Institut), Munich, Germany

${ }^{101}$ Nagasaki Institute of Applied Science, Nagasaki, Japan

102 Graduate School of Science and Kobayashi-Maskawa Institute, Nagoya University, Nagoya, Japan

103 (a) INFN Sezione di Napoli, Naples, Italy; (b) Dipartimento di Fisica, Università di Napoli, Naples, Italy

${ }^{104}$ Department of Physics and Astronomy, University of New Mexico, Albuquerque, NM, USA

105 Institute for Mathematics, Astrophysics and Particle Physics, Radboud University Nijmegen/Nikhef, Nijmegen, The Netherlands

${ }^{106}$ Nikhef National Institute for Subatomic Physics and University of Amsterdam, Amsterdam, The Netherlands

${ }^{107}$ Department of Physics, Northern Illinois University, DeKalb, IL, USA

108 Budker Institute of Nuclear Physics, SB RAS, Novosibirsk, Russia

${ }^{109}$ Department of Physics, New York University, New York, NY, USA

110 Ohio State University, Columbus, OH, USA

${ }^{111}$ Faculty of Science, Okayama University, Okayama, Japan

${ }^{112}$ Homer L. Dodge Department of Physics and Astronomy, University of Oklahoma, Norman, OK, USA

113 Department of Physics, Oklahoma State University, Stillwater, OK, USA

${ }^{114}$ Palacký University, RCPTM, Olomouc, Czech Republic

115 Center for High Energy Physics, University of Oregon, Eugene, OR, USA

${ }^{116}$ LAL, Université Paris-Sud and CNRS/IN2P3, Orsay, France

${ }^{117}$ Graduate School of Science, Osaka University, Osaka, Japan

${ }^{118}$ Department of Physics, University of Oslo, Oslo, Norway

${ }^{119}$ Department of Physics, Oxford University, Oxford, UK

120 (a) INFN Sezione di Pavia, Pavia, Italy; ${ }^{\text {(b) }}$ Dipartimento di Fisica, Università di Pavia, Pavia, Italy

${ }^{121}$ Department of Physics, University of Pennsylvania, Philadelphia, PA, USA

122 Petersburg Nuclear Physics Institute, Gatchina, Russia

123 (a) INFN Sezione di Pisa, Pisa, Italy; (b) Dipartimento di Fisica E. Fermi, Università di Pisa, Pisa, Italy

${ }^{124}$ Department of Physics and Astronomy, University of Pittsburgh, Pittsburgh, PA, USA

125 (a) Laboratorio de Instrumentacao e Fisica Experimental de Particulas-LIP, Lisbon, Portugal; (b) Faculdade de Ciências, Universidade de Lisboa, Lisbon, Portugal; (c) Department of Physics, University of Coimbra, Coimbra, Portugal;

(d) Centro de Física Nuclear da Universidade de Lisboa, Lisbon, Portugal; (e) Departamento de Fisica, Universidade do Minho, Braga, Portugal; ${ }^{(\mathrm{f})}$ Departamento de Fisica Teorica y del Cosmos and CAFPE, Universidad de Granada, Granada, Spain; ${ }^{(g)}$ Dep Fisica and CEFITEC of Faculdade de Ciencias e Tecnologia, Universidade Nova de Lisboa, Caparica, Portugal

${ }^{126}$ Institute of Physics, Academy of Sciences of the Czech Republic, Prague, Czech Republic

${ }^{127}$ Czech Technical University in Prague, Prague, Czech Republic

${ }^{128}$ Faculty of Mathematics and Physics, Charles University in Prague, Prague, Czech Republic

${ }^{129}$ State Research Center Institute for High Energy Physics, Protvino, Russia

${ }^{130}$ Particle Physics Department, Rutherford Appleton Laboratory, Didcot, UK

${ }^{131}$ Physics Department, University of Regina, Regina, SK, Canada

132 Ritsumeikan University, Kusatsu, Shiga, Japan

133 (a) INFN Sezione di Roma, Rome, Italy; (b) Dipartimento di Fisica, Sapienza Università di Roma, Rome, Italy 
134 (a) INFN Sezione di Roma Tor Vergata, Rome, Italy; (b) Dipartimento di Fisica, Università di Roma Tor Vergata, Rome, Italy

135 (a) INFN Sezione di Roma Tre, Rome, Italy; (b) Dipartimento di Matematica e Fisica, Università Roma Tre, Rome, Italy

136 (a) Faculté des Sciences Ain Chock, Réseau Universitaire de Physique des Hautes Energies-Université Hassan II, Casablanca, Morocco; (b) Centre National de l'Energie des Sciences Techniques Nucleaires, Rabat, Morocco; (c) Faculté des Sciences Semlalia, Université Cadi Ayyad, LPHEA-Marrakech, Marrakech, Morocco; (d) Faculté des Sciences, Université Mohamed Premier and LPTPM, Oujda, Morocco; (e) Faculté des Sciences, Université Mohammed V-Agdal, Rabat, Morocco

137 DSM/IRFU (Institut de Recherches sur les Lois Fondamentales de l'Univers), CEA Saclay (Commissariat à l'Energie Atomique et aux Energies Alternatives), Gif-sur-Yvette, France

138 Santa Cruz Institute for Particle Physics, University of California Santa Cruz, Santa Cruz, CA, USA

139 Department of Physics, University of Washington, Seattle, WA, USA

140 Department of Physics and Astronomy, University of Sheffield, Sheffield, UK

141 Department of Physics, Shinshu University, Nagano, Japan

142 Fachbereich Physik, Universität Siegen, Siegen, Germany

143 Department of Physics, Simon Fraser University, Burnaby, BC, Canada

144 SLAC National Accelerator Laboratory, Stanford, CA, USA

145 (a) Faculty of Mathematics, Physics and Informatics, Comenius University, Bratislava, Slovak Republic; (b) Department of Subnuclear Physics, Institute of Experimental Physics of the Slovak Academy of Sciences, Kosice, Slovak Republic

146 (a) Department of Physics, University of Cape Town, Cape Town, South Africa; (b) Department of Physics, University of Johannesburg, Johannesburg, South Africa; (c) School of Physics, University of the Witwatersrand, Johannesburg,

South Africa

147 (a) Department of Physics, Stockholm University, Stockholm, Sweden; (b) The Oskar Klein Centre, Stockholm, Sweden

148 Physics Department, Royal Institute of Technology, Stockholm, Sweden

149 Departments of Physics and Astronomy and Chemistry, Stony Brook University, Stony Brook, NY, USA

${ }^{150}$ Department of Physics and Astronomy, University of Sussex, Brighton, UK

${ }^{151}$ School of Physics, University of Sydney, Sydney, Australia

152 Institute of Physics, Academia Sinica, Taipei, Taiwan

153 Department of Physics, Technion, Israel Institute of Technology, Haifa, Israel

154 Raymond and Beverly Sackler School of Physics and Astronomy, Tel Aviv University, Tel Aviv, Israel

155 Department of Physics, Aristotle University of Thessaloniki, Thessaloniki, Greece

156 International Center for Elementary Particle Physics and Department of Physics, The University of Tokyo, Tokyo, Japan

157 Graduate School of Science and Technology, Tokyo Metropolitan University, Tokyo, Japan

158 Department of Physics, Tokyo Institute of Technology, Tokyo, Japan

159 Department of Physics, University of Toronto, Toronto, ON, Canada

160 (a) TRIUMF, Vancouver, BC, Canada; (b) Department of Physics and Astronomy, York University, Toronto, ON, Canada

161 Faculty of Pure and Applied Sciences, University of Tsukuba, Tsukuba, Japan

162 Department of Physics and Astronomy, Tufts University, Medford, MA, USA

163 Centro de Investigaciones, Universidad Antonio Narino, Bogota, Colombia

164 Department of Physics and Astronomy, University of California Irvine, Irvine, CA, USA

165 (a) INFN Gruppo Collegato di Udine, Sezione di Trieste, Udine, Italy; ${ }^{(b)}$ ICTP, Trieste, Italy; ${ }^{\text {(c) }}$ Dipartimento di Chimica, Fisica e Ambiente, Università di Udine, Udine, Italy

166 Department of Physics, University of Illinois, Urbana, IL, USA

167 Department of Physics and Astronomy, University of Uppsala, Uppsala, Sweden

${ }^{168}$ Instituto de Física Corpuscular (IFIC) and Departamento de Física Atómica, Molecular y Nuclear and Departamento de Ingeniería Electrónica and Instituto de Microelectrónica de Barcelona (IMB-CNM), University of Valencia and CSIC, Valencia, Spain

169 Department of Physics, University of British Columbia, Vancouver, BC, Canada

170 Department of Physics and Astronomy, University of Victoria, Victoria, BC, Canada

${ }^{171}$ Department of Physics, University of Warwick, Coventry, UK

172 Waseda University, Tokyo, Japan

173 Department of Particle Physics, The Weizmann Institute of Science, Rehovot, Israel

174 Department of Physics, University of Wisconsin, Madison, WI, USA 
${ }^{175}$ Fakultät für Physik und Astronomie, Julius-Maximilians-Universität, Würzburg, Germany

${ }^{176}$ Fachbereich C Physik, Bergische Universität Wuppertal, Wuppertal, Germany

177 Department of Physics, Yale University, New Haven, CT, USA

178 Yerevan Physics Institute, Yerevan, Armenia

${ }^{179}$ Centre de Calcul de l'Institut National de Physique Nucléaire et de Physique des Particules (IN2P3), Villeurbanne, France

a Also at Department of Physics, King's College London, London, UK

${ }^{\mathrm{b}}$ Also at Institute of Physics, Azerbaijan Academy of Sciences, Baku, Azerbaijan

c Also at Particle Physics Department, Rutherford Appleton Laboratory, Didcot, UK

d Also at TRIUMF, Vancouver, BC, Canada

e Also at Department of Physics, California State University, Fresno, CA, USA

${ }^{\mathrm{f}}$ Also at Tomsk State University, Tomsk, Russia

g Also at CPPM, Aix-Marseille Université and CNRS/IN2P3, Marseille, France

h Also at Università di Napoli Parthenope, Naples, Italy

${ }^{i}$ Also at Institute of Particle Physics (IPP), Victoria, Canada

j Also at Department of Physics, St. Petersburg State Polytechnical University, St. Petersburg, Russia

k Also at Chinese University of Hong Kong, Hong Kong, China

${ }^{1}$ Also at Department of Financial and Management Engineering, University of the Aegean, Chios, Greece

$\mathrm{m}$ Also at Louisiana Tech University, Ruston, LA, USA

${ }^{\mathrm{n}}$ Also at Institucio Catalana de Recerca i Estudis Avancats, ICREA, Barcelona, Spain

${ }^{\circ}$ Also at Department of Physics, The University of Texas at Austin, Austin, TX, USA

p Also at Institute of Theoretical Physics, Ilia State University, Tbilisi, Georgia

${ }^{q}$ Also at CERN, Geneva, Switzerland

${ }^{\mathrm{r}}$ Also at Ochadai Academic Production, Ochanomizu University, Tokyo, Japan

${ }^{s}$ Also at Manhattan College, New York, NY, USA

${ }^{t}$ Also at Novosibirsk State University, Novosibirsk, Russia

u Also at Institute of Physics, Academia Sinica, Taipei, Taiwan

${ }^{v}$ Also at LAL, Université Paris-Sud and CNRS/IN2P3, Orsay, France

${ }^{w}$ Also at Academia Sinica Grid Computing, Institute of Physics, Academia Sinica, Taipei, Taiwan

${ }^{\mathrm{x}}$ Also at Laboratoire de Physique Nucléaire et de Hautes Energies, UPMC and Université Paris-Diderot and CNRS/IN2P3, Paris, France

${ }^{y}$ Also at School of Physical Sciences, National Institute of Science Education and Research, Bhubaneswar, India

${ }^{\mathrm{z}}$ Also at Dipartimento di Fisica, Sapienza Università di Roma, Rome, Italy

aa Also at Moscow Institute of Physics and Technology State University, Dolgoprudny, Russia

ab Also at Section de Physique, Université de Genève, Geneva, Switzerland

${ }^{a c}$ Also at International School for Advanced Studies (SISSA), Trieste, Italy

${ }^{a d}$ Also at Department of Physics and Astronomy, University of South Carolina, Columbia, SC, USA

ae Also at School of Physics and Engineering, Sun Yat-sen University, Guangzhou, China

af Also at Faculty of Physics, M.V. Lomonosov Moscow State University, Moscow, Russia

ag Also at Moscow Engineering and Physics Institute (MEPhI), Moscow, Russia

ah Also at Institute for Particle and Nuclear Physics, Wigner Research Centre for Physics, Budapest, Hungary

ai Also at Department of Physics, Oxford University, Oxford, UK

aj Also at Department of Physics, Nanjing University, Jiangsu, China

ak Also at Institut für Experimentalphysik, Universität Hamburg, Hamburg, Germany

al Also at Department of Physics, The University of Michigan, Ann Arbor, MI, USA

am Also at Discipline of Physics, University of KwaZulu-Natal, Durban, South Africa

an Also at University of Malaya, Department of Physics, Kuala Lumpur, Malaysia

* Deceased 